Ann. Scient. Éc. Norm. Sup.,

$4^{\mathrm{e}}$ série, t. 40, 2007, p. 387 à 444.

\title{
MODULI OF OBJECTS IN DG-CATEGORIES
}

\author{
By BERTRAND TOËN AND Michel VAQUIÉ
}

\begin{abstract}
The purpose of this work is to prove the existence of an algebraic moduli classifying objects in a given triangulated category.

To any dg-category $T$ (over some base ring $k$ ), we define a $D^{-}$-stack $\mathcal{M}_{T}$ in the sense of [TOËN B., Vezzosi G., Homotopical algebraic geometry II: Geometric stacks and applications, Mem. Amer. Math. Soc., in press], classifying certain $T^{\mathrm{op}}$-dg-modules. When $T$ is saturated, $\mathcal{M}_{T}$ classifies compact objects in the triangulated category $[T]$ associated to $T$. The main result of this work states that under certain finiteness conditions on $T$ (e.g. if it is saturated) the $D^{-}$-stack $\mathcal{M}_{T}$ is locally geometric (i.e. union of open and geometric sub-stacks). As a consequence we prove the algebraicity of the group of auto-equivalences of saturated dg-categories. We also obtain the existence of reasonable moduli for perfect complexes on a smooth and proper scheme, as well as complexes of representations of a finite quiver.
\end{abstract}

(C) 2007 Published by Elsevier Masson SAS

RÉSUMÉ. - L'objectif de ce travail est de démontrer l'existence d'un espace des modules algébrique classifiant les objets dans une catégorie triangulée donnée.

Pour toute dg-catégorie $T$ (au-dessus d'un anneau de base $k$ ), nous définissons un $D^{-}$-champ $\mathcal{M}_{T}$ dans le sens de [TOËN B., VEZzOsI G., Homotopical algebraic geometry II: Geometric stacks and applications, Mem. Amer. Math. Soc., in press], qui classifie certains $T^{\mathrm{op}}$-dg-modules. Quand $T$ est saturée, $\mathcal{M}_{T}$ classifie les objets compacts dans la catégorie triangulée $[T]$ associée à $T$. Le résultat principal de ce travail énonce que, sous certaines conditions de finitude sur $T$ (par exemple si $T$ est saturée), le $D^{-}$-champ $\mathcal{M}_{T}$ est localement géométrique (i.e. réunion de sous-champs ouverts géométriques). Comme conséquence, nous démontrons l'algébricité du groupe des auto-équivalences des dg-catégories saturées. Nous obtenons aussi l'existence d'un espace des modules raisonnable pour les complexes parfaits sur un schéma propre et lisse, ainsi que pour les complexes de représentations de carquois finis.

(c) 2007 Published by Elsevier Masson SAS

\section{Introduction}

This is the first part of a research project whose purpose is the study of moduli spaces (or rather stacks) of objects in a triangulated category of geometric or algebraic origin (for instance the bounded derived category of coherent sheaves on an algebraic variety, or the bounded derived category of finite dimensional modules over an associative algebra). The purpose of the present work is to prove the existence of a reasonable moduli of objects in a given triangulated category satisfying some finiteness assumptions and possessing a dg-enhancement in the sense of [2].

\subsection{The notion of moduli of objects in a category}

Before describing the main definitions and results of this work we will briefly review the notion of the moduli of objects in a (non triangulated) linear category $C$. For such a category 
the problem consists of finding an algebraic variety $m_{C}$, or more generally an algebraic space or Artin stack, whose points are in a natural bijection with the set of isomorphism classes of objects in $C$. A first problem in order to construct $m_{C}$ is to define the associated moduli functor, or in other words to define the notion of a family of objects in $C$ parametrized by a given scheme $X$. When the moduli functor is defined a second problem consists of proving its representability, by a scheme, an algebraic space or an Artin stack, which is only expected to hold when the category $C$ satisfies some further finiteness assumptions. When it exists, such a geometric object $m_{C}$ will be called a moduli space of objects in $C$.

A well understood example is when $C=A$ - $\bmod$ is the category of finite dimensional modules over a finitely generated associative algebra $A$ (over some algebraically closed field $k$ ). The corresponding moduli functor is then the functor sending an affine scheme Spec $B$ to the groupoid of $A \otimes B$-modules which are projective of finite rank over $B$. A natural description of the moduli is then given by the following quotient

$$
\coprod_{n \in \mathbb{N}} \operatorname{Hom}_{k-a l g}\left(A, M_{n}(k)\right) / G l_{n}(k),
$$

where $M_{n}(k)$ is the algebra of $n$ by $n k$-valued matrices. One important observation here is that the action of $G l_{n}(k)$ on $\operatorname{Hom}_{k-a l g}\left(A, M_{n}(k)\right)$ is not free, as its stabilizers correspond to automorphism groups of objects in $C$. In particular, it is well known that the above quotient does not exist as an algebraic variety, but only as an algebraic (1-)stack in the sense of Artin. In Section 1 we will review this example in more details for the reader's convenience.

The main question addressed in this work is to generalize the previous construction of the moduli $m_{T}$ to the case where $T$ is now a triangulated category. Again, the problems of defining a reasonable moduli functor, as well as proving the existence of the moduli $m_{T}$ itself (as a higher stack, see below) are only expected to have a solution when $T$ does satisfy some further finiteness conditions. By analogy with the non-triangulated case, these finiteness assumptions state that $T$ is equivalent (as a triangulated category) to $D_{\text {parf }}(B)$, the derived category of finite dimensional dg-modules over some dg-algebra $B$ of finite type (see 2.4). Moreover, we will see below that the moduli $m_{T}$ can reasonably exist only in the setting of higher algebraic stacks. From an intuitive point of view our main result can be expressed as follows.

THEOREM 0.1. - Let $T$ be a triangulated category which is triangulated equivalent to $D_{\text {parf }}(B)$, the derived category of finite dimensional (dg-)modules over a (dg-)algebra $B$ of finite type. Then, there exists an algebraic $\infty$-stack (see below) $m_{T}$ whose points are in one-toone correspondence with isomorphism classes of objects in $T$.

An important remark is that the derived category $T$ of an abelian category $C$ might satisfy these assumptions even though $C$ itself does not (a typical example is $C=\operatorname{Coh}(X)$ the category of coherent sheaves on a smooth proper variety $X$ ). Therefore, our main result not only implies the existence of a moduli for objects in a triangulated category, but also provides a new method to construct moduli for objects in a given abelian category (as a moduli subspace in some $m_{T}$ ).

\subsection{Dg-categories and higher stacks}

Working with triangulated categories brings several technical problems, and it turns out that the theory of triangulated categories is too coarse for our purpose (already the problem of defining a reasonable moduli functor does not seem to have any solution in the setting of triangulated categories). A much more flexible theory is obtained by replacing triangulated categories by $d g$-categories whose theory behaves much smoothly (see $[14,30]$ ) and which will be used as a ground setting in this work.

$4^{\text {e }}$ SÉRIE - TOME $40-2007-\mathrm{N}^{\circ} 3$ 
A dg-category is a generalization of a linear category in the sense that it consists of a set of objects together with complexes of morphisms between two objects, and composition maps preserving the linear and differential structures. To any such dg-category $T$, we define its homotopy category $[T]$, which is the category having the same objects as $T$ and with $H^{0}(T(x, y))$ as set of morphisms between $x$ and $y$. An important remark is that all triangulated categories of geometric or algebraic origin are of the form $[T]$ for some natural dg-category $T$. Moreover, the triangulated structure on $[T]$ is then completely determined by $T$ alone (see e.g. $[2,14])$. The structure of a dg-category can then be considered as a refinement of the structure of a triangulated category.

Let now $T$ be a fixed dg-category. An object $x \in T$ possesses an automorphism group $A u t(x)$ which is by definition the group of automorphisms of $x$ as an object in the genuine category $[T]$. It is also possible to define higher automorphisms groups by defining the $i$-automorphism groups of $x$ to be $E x t^{1-i}(x, x):=H^{1-i}(T(x, x))$ (for all $i>1$ ). This definition can be justified as follows: the topological intuition suggests that a dg-category is some sort of higher categorical structure, whose objects are the objects of $T$, whose 1-morphisms from $x$ to $y$ are zero cycles in $T(x, y)$, whose 2-morphisms from $u: x \rightarrow y$ to $v: x \rightarrow y$ are elements $h \in T(x, y)_{-1}$ such that $d h=v-u$, and more generally whose $i$-morphisms are elements in $T(x, y)_{1-i}$. Following this intuition, the group of 2-automorphisms of an object $x$ is the group of homotopy classes of self-homotopies of the identity $i d \in T(x, x)_{0}$, which is nothing else than $\operatorname{Ext}^{-1}(x, x)$. More generally, the group of $i$-automorphisms of $x$ is the group of homotopy classes of self-homotopies of $0 \in T(x, x)_{1-i}$, which is $\operatorname{Ext} t^{1-i}(x, x)$.

Because of the presence of the higher automorphisms groups, the classifying space of objects in $T$ is no longer a groupoid (objects plus isomorphisms), but should rather be a higher groupoid. A topological model for this groupoid is a homotopy type $|T|$ whose set of connected components is the set of equivalence classes of objects in $T$, and such that

$$
\pi_{1}(|T|, x) \simeq \operatorname{Aut}_{[T]}(x, x), \quad \pi_{i}(|T|, x) \simeq \operatorname{Ext}^{1-i}(x, x) \quad \forall i>1 .
$$

In other words, the moduli of objects in $T$ is not expected to be representable by an algebraic 1 -stack, but only by an algebraic $n$-stack, or even $\infty$-stack, taking into account all these higher automorphisms groups. This explains our choice to work within the theory of $\infty$-stacks, simply called stacks in the sequel, for which a good model is the homotopy theory of simplicial presheaves over the big site of affine schemes endowed with the étale topology (i.e. functors from the category of commutative rings to simplicial sets satisfying a certain étale descent condition, see $[35, \S 2.1])$.

\section{3. $D^{-}$-stacks}

We have just seen that for an object $x$ in a dg-category $T$, the negative ext groups $\operatorname{Ext}^{1-i}(x, x)$, for $i>1$, encode the higher automorphisms groups of the object $x$. The positive ext groups $\operatorname{Ext}^{i}(x, x)$, for $i>0$, also have an interpretation in terms of derived deformation theory, and thus in terms of extended moduli spaces of objects in $T$ (in the sense of the DDT philosophy, see for example $[6,13,15,36]$ for references on the subject). Indeed, it is very reasonable to expect that the tangent space of the moduli of objects in $T$ at an object $x$ is $\operatorname{Ext}^{1}(x, x)$. In the same way, it is expected that the first obstruction space at the object $x$ is $\operatorname{Ext}^{2}(x, x)$. Following the derived deformation theory philosophy, $\operatorname{Ext}^{i}(x, x)$ should be interpreted as a higher obstruction space, and more generally the whole complex $T(x, x)[1]$ should be thought of as the virtual tangent space at the point $x$. The existence of this virtual tangent space suggests that the moduli of objects in $T$ is not only a stack but should also come equipped with a virtual, or derived, structure. A good 
formalism for these derived stacks is the theory of $D^{-}$-stacks ${ }^{1}$ in the sense of [35] (see also [36] for an introduction). In terms of moduli functors this means that our moduli of objects in $T$ is not only a functor from commutative rings to simplicial sets, but rather a functor from simplicial commutative rings to simplicial sets. As shown in $[34,35]$ there exists a reasonable homotopy theory of such functors, as well as a notion of geometric $D^{-}$-stack, and $n$-geometric $D^{-}$-stacks, obtained by successive gluing of affine objects with respect to some natural extension of the smooth topology from commutative rings to simplicial commutative rings. These $n$-geometric $D^{-}$-stacks are derived analogs, and generalizations, of algebraic higher stacks of C. Simpson (see [27] and [35, §2.1]), and are global counter-parts of the formal extended moduli spaces appearing in the derived deformation theory program (e.g. as presented in [6]).

As the category of commutative rings sits naturally in the category of simplicial commutative rings (they are the constant simplicial objects), the category of $D^{-}$-stacks contains as a full sub-category the category of stacks. Furthermore the two notions of being geometric are compatible with respect to this inclusion, and thus geometric $D^{-}$-stacks are truly generalizations of geometric stacks and in particular of Artin's algebraic stacks, algebraic spaces and schemes. Moreover, to any $D^{-}$-stack is associated its truncation, which is a stack representing its classical, or un-derived part, and obtained by killing the non-trivial derived structure (in terms of moduli functors by restricting functors on simplicial rings to rings). This truncation functor, which is right adjoint to the inclusion, preserves geometric objects, and thus proving that a $D^{-}$-stack is geometric also implies that its truncation (or its classical part) is itself geometric. Moreover, these truncations come naturally equipped with a virtual structure sheaf, remembering the existence of the $D^{-}$-stack itself, which is an important additional structure from which virtual constructions (e.g. virtual fundamental classes) can be obtained.

All along this work we will place ourselves in the context of $D^{-}$-stacks as we think this is the right context in which moduli problems should be considered, but also as this will simplify the proof of our main result which would probably be more complicated in the underived context as certain computations of (co)tangent complexes are involved.

\subsection{The $D^{-}$-stack $\mathcal{M}_{T}$}

We are now ready to present the main object of this work. For a dg-category $T$ over some base ring $k$, we are looking for a geometric $D^{-}$-stack which classifies objects in $T$. Our first observation is that it is not reasonable to expect the existence of a geometric object classifying exactly objects in $T$. On one side, a general dg-category $T$ can have too few objects, as for instance a deformation of an object in $T$ might not stay in $T$ anymore (e.g. if $T$ has a unique object). On the other side, $T$ can also have too many objects, such as objects of infinite type. Both of these facts prevent the moduli of objects in $T$ to be reasonably behaved.

Our solution to this problem is to first enlarge $T$ to the bigger dg-category $\widehat{T}$, consisting of all $T^{\mathrm{op}}$-dg-modules ( $\widehat{T}$ is the dg-analog of the presheaf category). The $D^{-}$-stack $\mathcal{M}_{T}$ will then be defined in a way that its points are in correspondence with objects in $\widehat{T}$ satisfying some finiteness conditions called pseudo-perfectness (see below). More precisely, we define a functor

$$
\begin{aligned}
\mathcal{M}_{T}: s k-C A l g & \rightarrow \operatorname{SSet} \\
A & \mapsto \operatorname{Map}\left(T^{\mathrm{op}}, \widehat{A}_{\mathrm{pe}}\right),
\end{aligned}
$$

where $s k$-CAlg is the category of simplicial commutative $k$-algebras, $\widehat{A}_{\text {pe }}$ is the dg-category of perfect A-modules (see $\$ 2.4)$, and $\operatorname{Map}\left(T^{\mathrm{op}}, \widehat{A}_{\mathrm{pe}}\right)$ is the mapping space of the model category

\footnotetext{
${ }^{1}$ The notation $D^{-}$is to remind the negative bounded derived category. As explained in $[36,35] D^{-}$-stacks are stacks relative to the category of negatively graded cochain complexes.

$4^{\text {e }}$ SÉRIE - TOME $40-2007-\mathrm{N}^{\circ} 3$
} 
of dg-categories. Using the main results of [30], the classical points of $\mathcal{M}_{T}$ can be described in the following way. For a commutative $k$-algebra $A$, the (derived) base change from $k$ to $A$ of $T$ is denoted by $T \otimes^{\mathbb{L}} A$, which is another dg-category over $k$. It is then possible to identify the simplicial set $\mathcal{M}_{T}(A)$ with the classifying space of $T^{\mathrm{op}} \otimes^{\mathbb{L}} A$-dg-modules whose underlying complex of $A$-modules is perfect, also called pseudo-perfect $T^{\mathrm{op}} \otimes^{\mathbb{L}} A$-dg-modules. Concretely, this means that there is a bijection between $\pi_{0}\left(\mathcal{M}_{T}(A)\right)$ and the set of quasi-isomorphism classes of pseudo-perfect $T^{\mathrm{op}} \otimes^{\mathbb{L}} A$-dg-modules. Furthermore, the higher homotopy groups of $\mathcal{M}_{T}(A)$ are given by

$$
\pi_{1}\left(\mathcal{M}_{T}(A), x\right) \simeq \operatorname{Aut}(x), \quad \pi_{i}\left(\mathcal{M}_{T}(A), x\right) \simeq \operatorname{Ext}^{1-i}(x, x) \quad \forall i>1,
$$

where the automorphisms and ext groups are computed in the homotopy category of $T^{\mathrm{op}} \otimes^{\mathbb{L}}$ $A$-dg-modules. This shows that $\mathcal{M}_{T}$ classifies algebraic families of pseudo-perfect $T^{\mathrm{op}}$-dg-modules. These descriptions remain also valid when $A$ is now a simplicial commutative $k$-algebra, but using the correct notions for $T^{\mathrm{op}} \otimes^{\mathbb{L}} A$ and perfect complexes of $A$-modules.

Under certain finiteness conditions on $T$, properness and smoothness (see Definition 2.4), being pseudo-perfect is equivalent to being perfect, and thus $\mathcal{M}_{T}$ becomes a moduli for perfect $T^{\mathrm{op}}$-dg-modules. In particular, when $T$ is furthermore saturated (i.e. all perfect $T^{\mathrm{op}}$-dg-modules are (quasi-)representable, see Definition 2.4), $\mathcal{M}_{T}$ is truly a moduli of objects in $T$.

Finally, let us mention that the construction $T \mapsto \mathcal{M}_{T}$ is shown to be the adjoint to the functor sending a $D^{-}$-stack $F$ to the dg-category $L_{\mathrm{pe}}(F)$ of perfect complexes on $F$ (see Proposition 3.4). This provides a universal property satisfied by $\mathcal{M}_{T}$, showing for instance that for any scheme $X$, a morphism of $D^{-}$-stacks $X \rightarrow \mathcal{M}_{T}$ is precisely the same thing as a morphism of dg-categories $T \rightarrow L_{\mathrm{pe}}(X)$.

\subsection{The results}

The main theorem of this work states that the $D^{-}$-stack $\mathcal{M}_{T}$ is locally geometric and locally of finite presentation (i.e. union of open sub- $D^{-}$-stacks which are $n$-geometric for some $n$ and locally of finite presentation), under the condition that $T$ is of finite type (see Definition 2.4). Precisely, being of finite type means that the triangulated category of all $T^{\mathrm{op}}$-dg-module can be written as the homotopy category of dg-module over a dg-algebra $B$, which is itself finitely presented in the sense of the homotopy theory of dg-algebras. An important result states that a smooth and proper dg-category is always of finite type (see Corollary 2.13), giving a systematic way of producing finite type dg-categories of geometric origins.

The main theorem of this work is then the following.

THEOREM 0.2 (Theorem 3.6, Corollary 3.17). - Let T be a dg-category of finite type (e.g. $T$ saturated). Then, the $D^{-}$-stack $\mathcal{M}_{T}$ is locally geometric and locally of finite presentation (i.e. union of open $n$-geometric sub- $D^{-}$-stacks locally of finite presentation). Moreover, for any pseudo-perfect $T^{\mathrm{op}}$-dg-module E, corresponding to a global point of $\mathcal{M}_{T}$, the tangent complex of $\mathcal{M}_{T}$ at $E$ is given by

$$
\mathbb{T}_{\mathcal{M}_{T}, E} \simeq \mathbb{R} \underline{\operatorname{Hom}}(E, E)[1] .
$$

In particular, if $E$ is quasi-representable by an object $x$ in $T$, then we have

$$
\mathbb{T}_{\mathcal{M}_{T}, E} \simeq T(x, x)[1] .
$$

As a corollary of the above theorem it follows that the truncation $t_{0} \mathcal{M}_{T}$, which corresponds to the un-derived part of $\mathcal{M}_{T}$, is itself a locally geometric stack in the sense of C. Simpson's higher 
algebraic stacks [27]. However, the object $\mathcal{M}_{T}$ contains additional and non-trivial informations, and is better behaved from an infinitesimal point of view, as is shown by the above formula for its tangent complex (note for instance that the tangent complex of the truncation is not known).

An interesting easy consequence of our main theorem is the following representability result.

COROLlARY 0.3 (Corollary 3.24). - Let $T$ be a saturated dg-category over some field $k$. Then, the group aut $(T)$ of auto-equivalences of $T$ is representable by a group scheme locally of finite type over $k$.

We will present two applications of Theorem 0.2. The first one is for a smooth and proper scheme $X \rightarrow$ Spec $k$, and $T$ the dg-category of perfect complexes on $X$. In this case, $\mathcal{M}_{T}$ can be identified with the $D^{-}$-stack $\mathbb{R} \operatorname{Perf}(X)$, of perfect complexes on $X$. The second one is for $T$ the dg-category of bounded complexes of finite dimensional representations of a finite quiver $Q$. In this second case, $\mathcal{M}_{T}$ can be identified with the $D^{-}$-stack $\mathbb{R} P \operatorname{erf}(Q)$ of pseudo-perfect complexes of representations of $Q$, which are nothing else that complexes of representations whose underlying complexes at each node is a perfect complex of $k$-modules. When $Q$ has no loops, $T$ is furthermore saturated and thus $\mathbb{R} \operatorname{Perf}(Q)$ is truly the $D^{-}$-stack of perfect complexes of representations of $Q$.

COROLlary 0.4 (Corollaries 3.29 and 3.31). - With the above notations, the $D^{-}$-stacks $\mathbb{R} \underline{\operatorname{Perf}}(X)$ and $\mathbb{R} \underline{\operatorname{Perf}}(Q)$ are locally geometric and locally of finite presentation.

Passing to the truncations of the $D^{-}$-stacks $\mathbb{R} \operatorname{Perf}(X)$ and $\mathbb{R} \operatorname{Perf}(Q)$ we obtain the following direct consequences, interesting for their own sake.

COROLlary 0.5. -

1. The stack of perfect complexes on a smooth and proper scheme is locally geometric.

2. The stack of pseudo-perfect complexes of representations of a finite quiver is locally geometric.

For a smooth and proper scheme $X$, it is easy to deduce from the above corollary that the usual 1-stack $\underline{\operatorname{Vect}}(X)$, of vector bundles on $X$ is representable by an Artin 1-stack locally of finite type. This provides a new construction of moduli of vector bundles on smooth proper schemes, which does not use Artin's representability criterion.

\subsection{Future and related works}

To start with, we would like to mention that the next step in our general study of the moduli $\mathcal{M}_{T}$ will be to describe the sub- $D^{-}$-stacks defined by the additional data of t-structures and stability conditions. We expect that any reasonable t-structure on a saturated dg-category $T$ will define an open sub- $D^{-}$-stack of perverse objects in $\mathcal{M}_{T}$, and thus will define a geometric $D^{-}$-stack whose truncation will be an algebraic 1-stack (e.g. in the sense of Artin). In the same way, semi-stable objects with respect to a reasonable stability condition should define an open sub- $D^{-}$-stack in $\mathcal{M}_{T}$, whose truncation will again be an algebraic 1-stack in the sense of Artin. Furthermore, a certain moduli of stable point-like objects is expected to be representable by a smooth and proper scheme $X$ mapping to $\mathcal{M}_{T}$. This will provide a general approach to the realization problem, asking whether or not it is possible to find a smooth and proper variety $X$ for which $T$ is equivalent to the dg-category $L_{\mathrm{pe}}(X)$ of perfect complexes on $X$, those $X$ being precisely realized as the moduli of point-like objects in $T$. We think to have a partial solution to this problem and we hope to come back to this question in a future work. A far reaching application to this construction would be to try to construct the mirror of a Calabi-Yau manifold $X$, as a moduli of point-like objects in the Fukaya category $F u k(X)$, Theorem 0.2 being the key statement insuring that this moduli is representable by another Calabi-Yau manifold $X^{\prime}$.

$4^{\mathrm{e}}$ SÉRIE - TOME $40-2007-\mathrm{N}^{\circ} 3$ 
In [31], (derived) Hall algebras of dg-categories have been introduced from a purely categorical approach. As for Hall algebras of abelian categories (see e.g. [12]), it is expected that these derived Hall algebras possess geometric counter-parts, defined in terms of convolution product on l-adic sheaves on the $D^{-}$-stack $\mathcal{M}_{T}$. A consequence of Theorem 0.2 is most probably that such geometric derived Hall algebras exist, but also that they map to the one of [31] by means of a faisceaux-fonctions correspondence and trace formula for higher stacks. These kinds of constructions and technologies are being studied and will appear in another work.

Smooth and proper dg-categories have been also studied from the point of view of noncommutative geometry "à la Kontsevich" (see [16]). The main result of this paper constructs from a smooth and proper dg-category, or in other words from a non-commutative algebraic manifold, a $D^{-}$-stack in the sense of [35], and therefore provides a link between non-commutative geometry and (commutative) derived algebraic geometry.

From the geometricity of the $D^{-}$-stack $\mathbb{R} \operatorname{Perf}(X)$, we easily deduce a positive answer to Conjecture 5.4 [36], stating that the $D^{-}$-stack $\overline{\mathbb{R} V e c t}_{n}(X)$ of vector bundles on $X$ is geometric (as $\mathbb{R} \operatorname{Vect}_{n}(X)$ is obviously an open sub- $D^{-}$-stack of $\mathbb{R} \operatorname{Perf}(X)$ ). We should acknowledge however that this conjecture has been proved previously by J. Gorski in his thesis [5], by completely different methods using a derived version of the Quot functor and valid for nonsmooth varieties.

Our main result and its consequence on the geometricity of the stack of perfect complexes (Corollary 0.5 (2)), recover and provide new proofs of the result of the recent paper [19] (and thus of [11]), at least in the smooth case. Indeed, the moduli functors considered in [19] are clearly an open sub-stack of the truncation of $\mathbb{R} \operatorname{Perf}(X)$ (precisely the one consisting of perfect complexes $E$ such that $E x t^{-i}(E, E)=0$ for all $\left.i>0\right)$, which is thus geometric by our Theorem 0.2 . Contrary to [19] our proof does not use Artin's representability criterion, and also provides descriptions of sub-stacks of finite type (see Corollary 3.32), which seem to be hard to obtain using the methods of [19]. Some further applications of the existence of these moduli spaces are given in $[1,33]$. Moreover, our theorem also provides a non-trivial derived structure on the stack considered in [19], and thus is a strictly stronger result (again in the smooth case). As an example of application, this derived structure can be used to prove very easily the existence of the virtual fundamental class used in [29], as done for instance at the very end of [4] for the stack of stable maps (see [32, §4.4, Ex. 4] for more details).

Finally, we think it is possible to weaken the finite type assumption on the dg-category $T$ and still be able to prove Theorem 0.2. A possible approach would probably be a direct application of Artin's representability criterion as well as its extension to the context of $D^{-}$-stacks by J. Lurie (see [20]). However, we think it is not that much easier to check that the conditions of application of this criterion are satisfied than to prove directly by hand that $\mathcal{M}_{T}$ is locally geometric. Moreover, our approach also provides a description of the sub- $D^{-}$-stacks of finite type in $\mathcal{M}_{T}$, which does not seem easy to obtain using Artin's representability criterion.

Another approach to generalize our Theorem 0.2 would be to use the theory of $D$-stacks of [35], which are generalizations of $D^{-}$-stacks $\left(D^{-}\right.$-stacks sit inside $D$-stacks in the same way as negatively graded complexes sit inside unbounded complexes ${ }^{2}$ ). Our method of proof of Theorem 0.2 shows that $\mathcal{M}_{T}$ is a 1-geometric $D$-stack (in the sense of [35]) as soon as $T$ has compact generator (however $\mathcal{M}_{T}$ is not locally finitely presented anymore). Since being geometric as a $D$-stack is strictly weaker than being geometric as a $D^{-}$-stack, this does not imply that our Theorem 0.2 stays correct under this very weak assumption. However, the difference between the two notions is somehow mild, and most probably these are equivalent for locally finitely presented objects.

\footnotetext{
${ }^{2}$ The notation $D$ is to remind the unbounded derived category.
} 
Conventions. - All along this work we will fix two universes $\mathbb{U} \in \mathbb{V}$. We will always assume that they satisfy the infinite axiom. By convention, all structures considered, such as sets, groups, rings ... will be elements of $\mathbb{U}$, except if the contrary is explicitly stated. Thus, SSets will denote the category of simplicial sets in $\mathbb{U}$, whereas $S S e t_{\mathbb{V}}$ will denote the category of simplicial sets in $\mathbb{V}$. The same notations will be used for other categories as well (e.g. Sets, Sets $\mathbb{V}$ ). The unique exception is with the word categories, that will not have to be elements of $\mathbb{U}$. Categories which are elements of $\mathbb{U}$ will be called small categories.

We use the notion of model categories in the sense of [9]. The expression equivalence always refers to weak equivalence in a model category. For a model category $M$, we will denote by $M a p_{M}$ (or Map if $M$ is clear) its mapping spaces as defined in [9]. In the same way, the set of morphisms in the homotopy category $H o(M)$ will be denoted by $[-,-]_{M}$, or by $[-,-]$ if $M$ is clear. The natural $\mathrm{Ho}(S S e t)$-tensor structure on $H o(M)$ will be denoted by $K \otimes^{\mathbb{L}} X$, for $K$ a simplicial set and $X$ an object in $M$. In the same way, the $H o(S S e t)$-cotensor structure will be denoted by $X^{\mathbb{R} K}$. The homotopy fiber products will be denoted by $x \times{ }_{z}^{h} y$, and dually the homotopy push-outs will be denoted by $x \amalg_{z}^{\mathbb{L}} y$.

For a simplicial set $X$, we say that a sub-simplicial set $Y \subset X$ is full, if $Y$ is a union of connected components of $X$. For two objects $x$ and $y$ in a model category $M$, we denote by $\operatorname{Map}_{M}^{\mathrm{eq}}(x, y)$ (or $\operatorname{Map}^{\mathrm{eq}}(x, y)$ if $M$ is clear) the full sub-simplicial set of $\operatorname{Map}_{M}(x, y)$ consisting of connected components corresponding to equivalences (i.e. corresponding to isomorphisms in $H o(M)$ through the identification between $\pi_{0}\left(\operatorname{Map}_{M}(x, y)\right)$ and $\left.[x, y]_{M}\right)$.

For all along this work, we fix an associative, unital and commutative ring $k$. We denote by $C(k)$ the category of (un-bounded) complexes of $k$-modules. Our complexes will always be cohomologically indexed (i.e. with increasing differentials). The shift of a complex $E$ is denoted by $E[1]$, and defined by $E[1]_{n}:=E_{n+1}$ (with the usual sign rule $d_{n}^{E[1]}:=-d_{n+1}^{E}$ ). The category $C(k)$ is a symmetric monoidal model category, using the projective model structures for which fibrations are epimorphisms and equivalences are quasi-isomorphisms (see e.g. [9]). The monoidal structure on $C(k)$ is the tensor product of complexes over $k$, and will be denoted by $\otimes$. Its derived version will be denoted by $\otimes^{\mathbb{L}}$.

Finally, for $B$ a dg-algebra over $k$, we denote by $B$-Mod its category of left dg-modules. In order to avoid confusions, when $B$ is a (non-dg) $k$-algebra, the category of (non-dg) left $B$-modules will be denoted by $\operatorname{Mod}(B)$, whereas $B$ - $\operatorname{Mod}$ will denote the category of left $B$-dgmodules (or in other words the category or complexes of left $B$-modules).

\section{The 1-stack of objects in a linear category}

The purpose of this first section is to quickly review a construction of the moduli 1-stack of objects in a linear category $C$. The construction of the moduli stack $\mathcal{M}_{T}$ of objects in a dg-category $T$ will be based on the same ideas, and we hope this section will help to understand the definitions and results of the sequel. However, this part is independent from the rest of the paper and the reader can skip it.

All along this first section the word stacks or 1-stacks refer to the usual notion, e.g. as presented in [17].

We fix a $k$-linear category $C$ and we define a moduli functor

$$
m_{C}: k-C A l g \rightarrow G p d,
$$

$4^{\text {e }}$ SÉRIE - TOME $40-2007-\mathrm{N}^{\circ} 3$ 
from the category of commutative $k$-algebras to the category of groupoids, in the following way. For $k^{\prime} \in k-C A l g$ we set

$$
m_{C}\left(k^{\prime}\right):=\operatorname{Fun}_{k}\left(C^{\mathrm{op}}, \operatorname{Mod}\left(k^{\prime}\right)^{\mathrm{proj}, \mathrm{tf}}\right)
$$

the groupoid of $k$-linear functors from $C$ to the category of $k^{\prime}$-modules which are projective of finite type. For $k^{\prime} \rightarrow k^{\prime \prime}$ a morphism in $k$-CAlg we have a base change functor

$$
k^{\prime \prime} \otimes_{k^{\prime}}-: \operatorname{Mod}\left(k^{\prime}\right)^{\text {proj,tf }} \rightarrow \operatorname{Mod}\left(k^{\prime \prime}\right)^{\text {proj,tf }},
$$

and thus a functor

$$
m_{C}\left(k^{\prime}\right) \rightarrow m_{C}\left(k^{\prime \prime}\right)
$$

obtained by composition. This defines a (lax) functor $m_{C}$ which is easily seen to be a 1-stack (on the big étale site of affine $k$-schemes).

We will say that $C$ is of finite type (as a $k$-linear category) when the $k$-linear category $\widehat{C}=\operatorname{Mod}\left(C^{\mathrm{op}}\right)$ of $C^{\mathrm{op}}$-modules is equivalent to $\operatorname{Mod}(B)$ for $B$ a finitely presented associative $k$-algebra. Equivalently, $C$ is of finite type if it is Morita equivalent to a finitely presented associative $k$-algebra $B$ (considered as a linear category with a unique object).

THEOREM 1.1. - With the above notation, and for $C$ a $k$-linear category of finite type, the 1-stack $m_{C}$ is an Artin stack locally of finite presentation.

The proof of the theorem starts by considering a morphism of stacks

$$
\pi: m_{C} \rightarrow m_{k}=\underline{\text { Vect }}
$$

where $m_{k}=\underline{\text { Vect }}$ is the stack of vector bundles, defined as follows. The choice of an equivalence $\widehat{C} \simeq \operatorname{Mod}(B)$, induces an equivalence between the stack $m_{C}$ and the stack $m_{B^{\text {op }}}$ of left $B$-modules which are projective and of finite type over the base $k$. In other words, the groupoid $m_{C}\left(k^{\prime}\right)$ is naturally equivalent to the groupoid of $B \otimes_{k} k^{\prime}$-modules which are projective and of finite type as $k^{\prime}$-modules. We define

$$
\pi\left(k^{\prime}\right): m_{C}\left(k^{\prime}\right) \rightarrow \underline{\operatorname{Vect}}\left(k^{\prime}\right)
$$

by sending a $B \otimes_{k} k^{\prime}$-module to its underlying $k^{\prime}$-module.

It is well known that the stack $\underline{\text { Vect }}$ is an Artin stack locally of finite type, and more precisely we have

$$
\underline{V e c t} \simeq \coprod_{n} B G l_{n}
$$

Therefore, to prove Theorem 1.1 it is enough to prove that the morphism $\pi$ is representable (we use a key fact that the domain of a representable morphism is an Artin stack if the codomain is so). Let $X=S p e c k^{\prime}$ be an affine $k$-scheme, and $X \rightarrow$ Vect a morphism corresponding to a $k^{\prime}$-module $M$ projective and of finite type. We consider the stack $m_{C} \times_{\underline{V e c t}} X$, which can be identified with the sheaf of $B$-module structures on $M$

$$
\begin{aligned}
\underline{\operatorname{Hom}}(B, \operatorname{End}(M)): k^{\prime}-C A l g & \rightarrow \text { Sets } \\
k^{\prime \prime} & \mapsto H_{o m} m_{k-A l g}\left(B, \operatorname{End}(M) \otimes_{k^{\prime}} k^{\prime \prime}\right) .
\end{aligned}
$$


The algebra $B$ being finitely presented, we can write $B$ as a push-out

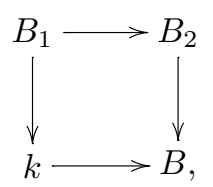

where $B_{i}$ are free associative algebras with a finite number of generators $n_{i}$. The sheaf $\underline{\operatorname{Hom}}(B, \operatorname{End}(M))$ can then be written as a pull-back

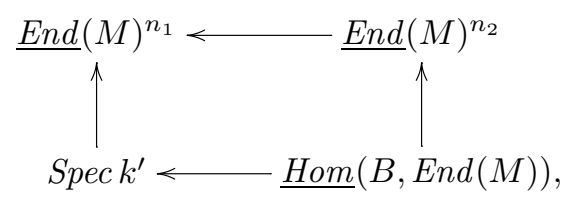

where $\operatorname{End}(M)$ is the sheaf of endomorphisms of the $k^{\prime}$-module $M$. The module $M$ being projective and of finite type the sheaf $\underline{E n d}(M)$ is representable by an affine $k^{\prime}$-scheme of finite presentation, and thus $\underline{\operatorname{Hom}}(B, \operatorname{End}(M))$ is also an affine $k^{\prime}$-scheme of finite presentation. This finishes the sketch of a proof of the theorem.

The stack $m_{C}$ is rather far from the starting category $C$ itself in general, as its groupoid of global points $m_{C}(k)$ can be quite different from the groupoid of isomorphisms in $C$ (in general, there is not even a natural functor between these two groupoids). However, under some conditions on $C$ it can be shown to actually classify objects in $C$. For this we will say that $C$ is locally proper if its Hom's are projective $k$-module of finite type. We will say that $C$ is smooth if $C$ is a projective $C \otimes_{k} C^{\mathrm{op}}$-module of finite type. We say that $C$ has a compact and projective generator if it is Morita equivalent to an associative $k$-algebra $B$. Finally, we say that $C$ is saturated if it is Karoubian, smooth, proper and has a compact and projective generator. It can be shown that $C$ is saturated if and only if it is equivalent to the category $\operatorname{Mod}(B)^{\text {proj,tf }}$, of projective $B$-modules of finite type, for $B$ an associative $k$-algebra satisfying the following two conditions:

- $B$ is projective of finite type as a $k$-module.

- $B$ is a projective $B \otimes_{k} B^{\mathrm{op}}$-module of finite type.

Remark 1.2. - The important two properties of saturated $k$-linear categories are the following.

- Any saturated $k$-linear category $C$ is of finite type, and thus $m_{C}$ is an Artin stack.

- The groupoid of global points $m_{C}(k)$ is equivalent to the groupoid of objects in $C$. More precisely, $m_{C}\left(k^{\prime}\right)$ is equivalent to the groupoid of $k^{\prime}$-modules in $C$ (i.e. $k$-linear functors $k^{\prime} \rightarrow C$ ). Therefore, $m_{C}$ is truly a moduli of objects in $C$.

In the sequel we will formally follow the same ideas. For this we will start by replacing $k$-linear categories by $k$-dg-categories, associative $k$-algebras by associative $k$-dg-algebras, modules by dg-modules ... and so on. Our first task will be to introduce analogs of the notions of being of finite type, projective of finite type, smooth, proper ... suited in the dg world. With these notions, the definition of the moduli stack $\mathcal{M}_{T}$ for a dg-category $T$ will be very similar to the definition of the 1-stack $m_{C}$, and the proof of its algebraicity will be very close to the proof of Theorem 1.1 presented above.

It should be noted that being saturated is a very strong, and thus not very convenient condition on a linear category. However, the analogous notion for dg-categories will be much more interesting as it will be satisfied in many examples (e.g. an algebra might be saturated when considered as a dg-category without being saturated as a linear category).

$4^{\text {e }}$ SÉRIE - TOME $40-2007-\mathrm{N}^{\circ} 3$ 


\section{Preliminaries}

In this section we have gathered definitions and results that will be used in the next section for the definition of the $D^{-}$-stack $\mathcal{M}_{T}$ and for the proof of its geometricity. The first paragraph on model categories seems to be of a folklore knowledge and is essentially extracted from [7]. In the second paragraph we review some terminology and results concerning dg-categories. Most of them are well known, but some of them are probably new. Finally, the last two paragraphs are extracted from [35], and contain the basic language of derived algebraic geometry we will need in the sequel. The notion of locally geometric $D^{-}$-stack is the only new notion which does not appear in [35], but is a straightforward generalization of the notion of $n$-geometric $D^{-}$-stacks.

\subsection{Compactly generated model categories}

We fix a model category $M$. The purpose of this paragraph is to consider the relations between homotopically finitely presented objects and finite cell objects, under certain conditions on $M$.

Let us fix $I$, a set of generating cofibrations in $M$.

DEFINITION 2.1.-

1. An object $X$ is a strict finite $I$-cell object, if there exist a finite sequence

$$
X_{0}=\emptyset \longrightarrow X_{1} \longrightarrow \cdots \longrightarrow X_{n}=X
$$

and for any $0 \leqslant i<n$ a push-out square

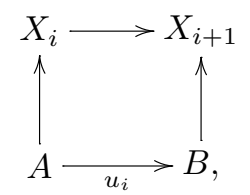

with $u_{i} \in I$.

2. An object $X$ is a finite $I$-cell object (or simply a finite cell object when $I$ is clear) if it is equivalent to a strict finite $I$-cell object.

3. An object $X \in M$ is homotopically finitely presented if for any filtered system of objects $Y_{i}$ in $M$, the natural morphism

$$
\operatorname{Colim}_{i} \operatorname{Map}\left(X, Y_{i}\right) \rightarrow \operatorname{Map}\left(X, \operatorname{Hocolim}_{i} Y_{i}\right)
$$

is an isomorphism in $\mathrm{Ho}($ SSet $)$.

4. The model category $M$ is compactly generated, if it is cellular (in the sense of [7, §12]), and there is a set of generating cofibrations $I$ and generating trivial cofibrations $J$ whose domains and codomains are cofibrant, $\omega$-compact, $\omega$-small (with respect to the whole category $M$ ).

The main result of this paragraph is the following proposition. It seems to be a folklore result, but for which we do not know any reference.

PROPOSITION 2.2. - Let $M$ be a compactly generated model category, and I be a set of generating cofibrations whose domains and codomains are cofibrant, $\omega$-compact and $\omega$-small with respect to the whole category $M$.

1. A filtered colimit of fibrations (resp. trivial fibrations) is a fibration (resp. a trivial fibration). 
2. For any filtered diagram $X_{i}$ in $M$, the natural morphism

$$
\operatorname{Hocolim}_{i} X_{i} \rightarrow \operatorname{Colim}_{i} X_{i}
$$

is an isomorphism in $\mathrm{Ho}(M)$.

3. Any object $X$ in $M$ is equivalent to a filtered colimit of strict finite I-cell object.

4. If furthermore, filtered colimits are exact in $M$, an object $X$ in $M$ is homotopically finitely presented if and only if it is equivalent to a retract in $\mathrm{Ho}(M)$ of a strict finite I-cell object.

Proof. - (1) Note first that by definition $M$ is finitely generated in the sense of [9, §7]. Property (1) is then proved in $[9, \S 7.4 .1]$.

(2) As homotopy colimit is the left derived functor of colimit, it is enough to show that filtered colimits preserve equivalences. For a filtered category $A$, the functor colim: $M^{A} \rightarrow M$ is left Quillen for the projective model structure (equivalences and fibrations are levelwise). By (1), colim preserves trivial fibrations. Therefore, the functor colim is a left Quillen functor preserving trivial fibrations, and thus preserves equivalences.

(3) The small object argument gives that any object $X$ is equivalent to a $I$-cell complex $Q(X)$. By $\omega$-compactness of the domains and codomains of $I, Q(X)$ is the filtered colimit of its finite sub- $I$-cell complexes. This implies that $X$ is equivalent to a filtered colimit of strict finite $I$-cell objects.

(4) Let $A$ be a filtered category, and $Y \in M^{A}$ be a $A$-diagram. Let $c(Y) \rightarrow R_{*}(Y)$ be a Reedy fibrant replacement of the constant simplicial object $c(Y)$ with values $Y$ (in the model category of simplicial objects in $M^{A}$, see $\left.[9, \S 5.2]\right)$. By (2), the induced morphism

$$
c\left(\operatorname{Colim}_{a \in A} Y_{a}\right) \rightarrow \operatorname{Colim}_{a \in A} R_{*}\left(Y_{a}\right)
$$

is an equivalence of simplicial objects in $M^{A}$. Moreover, (1) and the exactness of filtered colimit imply that $\operatorname{Colim}_{a \in A} R_{*}\left(Y_{a}\right)$ is a Reedy fibrant object in the model category of simplicial objects in $M$ (as filtered colimits commute with matching objects for the Reedy category $\Delta^{\mathrm{op}}$, see [9, $\S 5.2])$. This implies that for any cofibrant and $\omega$-small object $K$ in $M$, we have

$$
\begin{aligned}
\operatorname{Hocolim}_{a \in A} \operatorname{Map}\left(K, Y_{a}\right) & \simeq \operatorname{Colim}_{a \in A} \operatorname{Map}\left(K, Y_{a}\right) \simeq \operatorname{Colim}_{a \in A} \operatorname{Hom}\left(K, R_{*}\left(Y_{a}\right)\right) \\
& \simeq \operatorname{Hom}\left(K, \operatorname{Colim}_{a \in A} R_{*}\left(Y_{a}\right)\right) \simeq \operatorname{Map}\left(K, \operatorname{Colim}_{a \in A} Y_{a}\right) .
\end{aligned}
$$

This implies that the domains and codomains of $I$ are homotopically finitely presented.

As filtered colimits of simplicial sets preserve homotopy pull-backs, we deduce that any finite cell object is also homotopically finitely presented, as they are constructed from domains and codomains of $I$ by iterated homotopy push-outs (we use here that domains and codomains of $I$ are cofibrant). This implies that any retract of a finite cell object is homotopically finitely presented. Conversely, let $X$ be a homotopically finitely presented object in $H o(M)$, and by (3) let us write it as $\operatorname{Colim}_{i} X_{i}$, where $X_{i}$ is a filtered diagram of finite cell objects. Then, $[X, X] \simeq \operatorname{Colim}_{i}\left[X, X_{i}\right]$, which implies that this identity of $X$ factors through some $X_{i}$, or in other words that $X$ is a retract in $H o(M)$ of some $X_{i}$.

Examples of compactly generated model categories include $C(k)$ the model category of complexes of $k$-modules, $d g$-Cat the model category of dg-categories, and $T$-Mod the model category of dg-modules over a dg-category $T$ (see next paragraph).

\section{2. dg-categories}

We will use notations and definitions from [30] and [28]. We refer the reader to the overview [14] for an introduction to the concepts discussed in this paragraph.

$4^{\text {e }}$ SÉRIE - TOME $40-2007-\mathrm{N}^{\circ} 3$ 
The category of (small) dg-categories $d g$-Cat (over the base ring $k$ ) has a model structure for which equivalences are the quasi-equivalences (see [28]). The model category $d g$-Cat is furthermore compactly generated in the sense of 2.1 (4), as this can be seen easily using the standard generating cofibrations and trivial cofibrations of [28]. We recall from [30], that the homotopy category $\mathrm{Ho}(d g-\mathrm{Cat})$ has a natural symmetric monoidal structure $\otimes^{\mathbb{L}}$, induced by deriving the tensor product of dg-categories. This symmetric monoidal structure is known to be closed, and its corresponding internal Hom's will be denoted by $\mathbb{R} \underline{H o m}$ (see [30]).

Let $M$ be a $C(k)$-model category which we assume to be $\mathbb{V}$-small. We define a $\mathbb{V}$-small dg-category $\operatorname{Int}(M)$ in the following way. The set of objects of $\operatorname{Int}(M)$ is the set of fibrant and cofibrant objects in $M$. For two such objects $F$ and $E$ we set

$$
\operatorname{Int}(M)(E, F):=\underline{\operatorname{Hom}}(E, F) \in C(k),
$$

where $\underline{\operatorname{Hom}}(E, F)$ are the $C(k)$-valued Hom's of the category $M$. The set of objects of the dg-category $\operatorname{Int}(M)$ belongs to $\mathbb{V}$ but not to $\mathbb{U}$ anymore, and thus $\operatorname{Int}(M)$ is only a $\mathbb{V}$-small dgcategory. However, for any $E$ and $F$ in $\operatorname{Int}(M)$ the complex $\operatorname{Int}(M)(E, F)$ is in fact $\mathbb{U}$-small.

For $T$ a dg-category, we denote by $T^{\mathrm{op}}$ - $M o d$ the category of $T^{\mathrm{op}}$-dg-modules, i.e. the category of $C(k)$-enriched functors $F: T^{\mathrm{op}} \rightarrow C(k)$. The category $T^{\mathrm{op}}$-Mod can be endowed with a structure of model category such that a morphism $f: F \rightarrow G$ is an equivalence (resp. a fibration) if for any $z \in T$ the morphism $f_{z}: F(z) \rightarrow G(z)$ is an equivalence (resp. a fibration) in $C(k)$. Moreover the natural structure of $C(k)$-module on $T^{\mathrm{op}}$-Mod makes it a $C(k)$-model category in the sense of $[9,4.2 .18]$. The model category $T^{\mathrm{op}}$ - $M o d$ is compactly generated in the sense of Definition 2.1 (4), as this is easily seen using the natural generating cofibrations and trivial cofibrations (see e.g. [7, 11.6]).

To any dg-algebra $B$ (over $k$ ), we associate a dg-category, denoted also by $B$, with a unique object $*$ and such that the dg-algebra of endomorphisms of this object $B(*, *)$, is equal to $B$. The category $B$-Mod is then naturally isomorphic to the category of left- $B$-dg-modules. The dgcategory associated to the dg-algebra $k$ itself is denoted by $\mathbf{1}$, and is the dg-category with a unique object $*$ and with $\mathbf{1}(*, *)=k$. The dg-category $\mathbf{1}$ is also the unit for the monoidal structure $\otimes$ on $d g$-Cat. We recall our convention that for a non-dg $k$-algebra, considered as dg-category as above, $B$-Mod is then the category of complexes of left $B$-modules, and should not be confused with $\operatorname{Mod}(B)$ the category of left $B$-modules.

This construction provides a functor

$$
G: d g-A l g \rightarrow d g-C a t_{*}:=\mathbf{1} / d g-C a t,
$$

from the model category of associative and unital dg-algebras over $k$ (fibrations are epimorphisms and equivalences are quasi-isomorphisms, see e.g. [23]), to the model category of pointed dg-categories. This functor is easily seen to be a left Quillen functor, whose right adjoint sends a pointed dg-category $T$ to the dg-algebra $T(t, t)$ of endomorphisms of the distinguished object $t$ of $T$.

The $C(k)$-enriched version of the Yoneda lemma provides a morphism of dg-categories

$$
\underline{h}_{-}: T \rightarrow T^{\mathrm{op}}-\mathrm{Mod},
$$

defined by $\underline{h}_{x}(z)=T(z, x)$ which is quasi-fully faithful. This morphism is a morphism of $\mathbb{V}$-small dg-categories. As any object in $T^{\mathrm{op}}-$ Mod is fibrant, $\operatorname{Int}\left(T^{\mathrm{op}}-M o d\right)$ is simply the full sub- $C(k)$-enriched category of $T^{\mathrm{op}}$-Mod consisting of cofibrant objects, and we will denote it by $\widehat{T}$. In particular, the dg-category $\widehat{\mathbf{1}}$ is the dg-category of cofibrant complexes of $k$-modules. 
There is a natural isomorphism in $\mathrm{Ho}(\mathrm{dg}-\mathrm{Cat})$

$$
\widehat{T} \simeq \mathbb{R} \underline{\operatorname{Hom}}\left(T^{\mathrm{op}}, \widehat{\mathbf{1}}\right) .
$$

It is easy to see that for any $x \in T$ the object $\underline{h}_{x}$ is cofibrant in $T^{\mathrm{op}}-M o d$, and thus the morphism $\underline{h}$ factors as

$$
\underline{h}_{-}: T \rightarrow \widehat{T} .
$$

This last morphism is quasi-fully faithful, and by definition is the Yoneda embedding of the dg-category $T$.

DEFINITION 2.3. - Let $T$ be a dg-category. A $T^{\mathrm{op}}$-dg-module is perfect, or compact, if it is homotopically finitely presented in the model category $T^{\mathrm{op}}-$ Mod. The full sub-dg-category of perfect objects in $\widehat{T}$ will be denoted by $\widehat{T}_{\mathrm{pe}}$.

The model category $T^{\mathrm{op}}-M o d$ being compactly generated in the sense of Definition 2.1 (4), a $T^{\mathrm{op}}$-dg-module $F$ is in $\widehat{T}_{\mathrm{pe}}$ if and only if it is equivalent to a retract of some $F^{\prime}$, such that there exist a finite sequence

$$
F_{0}=0 \longrightarrow F_{1} \longrightarrow \cdots \longrightarrow F_{i} \longrightarrow F_{i+1} \longrightarrow \cdots \longrightarrow F_{n}=F^{\prime},
$$

and for any $i$ a (homotopy) push-out diagram

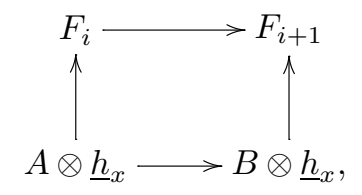

where $A \rightarrow B$ is a cofibration between bounded complexes of projective $k$-modules of finite type. Because of this description, up to a quasi-equivalence, the dg-category $\widehat{T}_{\mathrm{pe}}$ is small. In the sequel we will proceed as though $\widehat{T}_{\mathrm{pe}}$ were small. Note that for any $x \in T$, the $T^{\mathrm{op}}$-dg-module $\underline{h}_{x}$ is perfect, and therefore the Yoneda embedding factors as

$$
\underline{h}_{-}: T \rightarrow \widehat{T}_{\mathrm{pe}} \subset \widehat{T} .
$$

We also note that the above description of perfect $T^{\mathrm{op}}$-dg-modules implies that for a $k$-algebra $B$, considered as a dg-category, the perfect $B$-dg-modules are precisely the perfect complexes of $B$-modules (i.e. the complexes of $B$-modules which are quasi-isomorphic to bounded complexes of projective $B$-modules of finite presentation). This justifies the terminology perfect.

For any dg-category $T$ we denote by $[T]$ the category whose set of objects is the same as the one of $T$, and such that for any $x$ and $y$ in $T$ the set of morphisms is defined by $[T](x, y):=H^{0}(T(x, y))$ (with the natural induced composition maps). Note that for any $C(k)$ enriched model category $M$, there is a natural equivalence of categories $[\operatorname{Int}(M)] \simeq H o(M)$ ([30] Proposition 3.5). The construction $T \mapsto[T]$ is clearly functorial, and provides a functor from $d g$-Cat to categories, which furthermore sends quasi-equivalences to equivalences of categories.

For a dg-category $T$, the Yoneda embedding

$$
\underline{h}_{-}: T \rightarrow \widehat{T}
$$

$4^{\text {e }}$ SÉRIE - TOME $40-2007-\mathrm{N}^{\circ} 3$ 
induces a fully faithful functor

$$
\underline{h}_{-}:[T] \rightarrow\left[\widehat{T}_{\mathrm{pe}}\right] \subset[\widehat{T}] \simeq H_{o}\left(T^{\mathrm{op}}-M o d\right) .
$$

As $T^{\mathrm{op}}-\mathrm{Mod}$ is a stable model category in the sense of $[9, \S 7]$, there exists a natural triangulated structure on $\mathrm{Ho}\left(T^{\mathrm{op}}-\mathrm{Mod}\right)$ whose triangles are the image of the homotopy fibration sequences. The perfect $T^{\mathrm{op}}$-dg-modules are precisely the compact objects of this triangulated category in the sense of [21], and thus there exists a natural equivalence of triangulated categories

$$
\left[\widehat{T}_{\mathrm{pe}}\right] \simeq[\widehat{T}]_{c} .
$$

Moreover, $\left[\widehat{T}_{\text {pe }}\right] \subset[\widehat{T}]$ is the smallest thick triangulated sub-category (i.e. stable by shifts, cones and retracts) containing the image of $\underline{h}$. In other words, $\left[\widehat{T}_{\mathrm{pe}}\right]$ is the thick closure of $[T]$ in $[\widehat{T}]$. Using the language of dg-categories, this can also be stated as the fact that $\widehat{T}_{\mathrm{pe}}$ is the smallest full sub-dg-category or $\widehat{T}$ containing the essential image of $\underline{h}$, and which is stable by retracts, shifts and homotopy push-outs.

DEFINITION 2.4. - Let $T$ be a dg-category.

1. $T$ is locally perfect (or locally proper) if for any two objects $x$ and $y$ in $T, T(x, y)$ is a perfect complex of $k$-modules.

2. Thas a compact generator if the triangulated category $[\widehat{T}]$ has a compact generator (in the sense of [21]).

3. $T$ is proper if $T$ is locally perfect and has a compact generator.

4. $T$ is smooth if $T$, considered as a $T^{\mathrm{op}} \otimes^{\mathbb{L}} T$-dg-module

$$
\begin{aligned}
T: T^{\mathrm{op}} \otimes^{\mathbb{L}} T & \rightarrow C(k) \\
(x, y) & \mapsto T(x, y),
\end{aligned}
$$

is perfect as an object in $T \widehat{\otimes_{\mathbb{L}} T^{\mathrm{op}}}$.

5. A dg-category $T$ is triangulated if the Yoneda embedding

$$
\underline{h}: T \rightarrow \widehat{T}_{\mathrm{pe}}
$$

is a quasi-equivalence.

6. A dg-category $T$ is saturated if it is proper, smooth and triangulated.

7. A dg-category $T$ is of finite type if there exists a dg-algebra $B$, homotopically finitely presented in the model category $d g$ - $A l g$, and such that $\widehat{T}$ is quasi-equivalent to $\widehat{B^{\mathrm{OP}}}$.

The notions introduced in the previous definition are extensions to dg-categories of the notions we have used in our Section 1 for linear categories. The main idea was to think of the property of being perfect as a dg-version of the property of being projective and of finite type. Indeed, this analogy if based on the observation that projective modules of finite type over a commutative ring $k$ are exactly the dualizable objects in $\operatorname{Mod}(k)$. In the same way, the perfect complexes are exactly the dualizable objects in $D(k)$, the homotopy category of $\mathrm{dg}-k$-modules. With this as a starting point, the above definitions (1)-(4) are natural extensions of the notions presented in Section 1.

The notion of being triangulated is itself a dg-version of the notion of being Karoubian. In this sense, $\widehat{T}_{\text {pe }}$ is the triangulated hull of $T$ (see lemma below), in the same way as the Karoubian hull of a linear category $C$ is $\widehat{C}_{\text {proj,tf }}$ (the category of projective $C^{\text {op }}$-modules of finite type). 
The condition of being of finite type for the dg-category $T$ is the key property that will allow us to prove that the moduli stack $\mathcal{M}_{T}$ is geometric.

\section{Example 2.5. -}

1. Having a compact generator for a dg-category $T$ sounds like a strong condition, as the analog for linear categories is to be Morita equivalent to an algebra. The abelian category of quasi-coherent sheaves on a scheme cannot be written in general as the category of modules over an algebra. However, the dg-category of quasi-coherent complexes on any quasi-compact quasi-separated scheme is compactly generated (see [3, Thm. 3.1.1]). This is one of the many reasons why dg-categories of complexes of sheaves are in general better behaved than abelian category of sheaves.

The fact that the dg-categories of complexes of sheaves are compactly generated seems very specific to algebraic geometry. In fact, the dg-category of complexes of coherent sheaves on a smooth compact analytic variety is not compactly generated in general (see [3, Thm. 5.6.1]).

2. The smoothness notion of Definition 2.4 is an analog of the geometric notion for schemes. Indeed, if $A$ is a commutative algebra of finite type over a field $k$, then the morphism $\operatorname{Spec} A \rightarrow$ Spec $k$ is smooth if and only if the dg-category $\widehat{A}_{\text {pe }}$ of perfect complexes of $A$-modules is smooth in the sense of Definition 2.4. This notion of smoothness has been used by several authors in order to define non-commutative smooth varieties, (see e.g. [16]).

3. Our notion of being saturated does not seem exactly equivalent to the one defined for triangulated categories in [3]. It is closer to the notion of homologically smooth and compact $A_{\infty}$-algebra introduced in [16].

We think important to notice that being saturated as a dg-category is much weaker than being saturated as a linear category (as defined in $§ 1$ ). For instance, any finite dimensional $k$-algebra $B$ which is of finite global cohomological dimension is a saturated dg-category. In particular, the path algebra of a finite quiver without oriented loop is a saturated dgcategory but not a saturated linear category in general. We will also see that any smooth proper scheme has a saturated dg-category of perfect complexes (see §3.5).

4. In Definition 2.4 (7), we warn the reader that being of finite type must be understood in the derived and non-commutative sense. This notion is therefore rather far from the geometric notion of schemes of finite type (it is in fact stronger). Indeed, we will see that a dg-category of finite type is smooth (see 2.14). For instance, the commutative $k$-algebra $k[\epsilon]:=k[X] / X^{2}$, of dual numbers over $k$ is not of finite type as a dg-category.

The typical example of a homotopically finitely presented dg-algebra is given by a free dg-algebra (in the associative sense) over a finite number of generators. In fact, by Proposition 2.2 all homotopically finitely presented dg-algebras are obtained from the free dg-algebras over a finite number of generators by taking a finite number of homotopy pushouts and retracts. This fact will be used in the proof of our main theorem in order to reduce the problem to the free case.

The following lemma gathers some basic results concerning smooth and proper dg-categories. Some of them are most probably well-known folklore.

LEMMA 2.6. - Let T be a dg-category.

1. The dg-category $\widehat{T}_{\mathrm{pe}}$ is triangulated.

2. The dg-category $T$ has a compact generator (resp. is proper, resp. is smooth) if and only if $\widehat{T}_{\mathrm{pe}}$ has a compact generator (resp. is proper, resp. is smooth).

3. The dg-category $T$ has a compact generator if and only if there exists a dg-algebra $B$ such that $\widehat{T}$ and $\widehat{B^{\mathrm{OP}}}=\operatorname{Int}(B-M o d)$ are quasi-equivalent.

4e SÉRIE - TOME $40-2007-\mathrm{N}^{\circ} 3$ 
4. Suppose that $T$ has a compact generator and let $B$ be as in (3). Then, $T$ is proper if and only if the underlying complex of $B$ is perfect.

5. Suppose that $T$ has a compact generator and let $B$ be as in (3). The dg-category $T$ is smooth if and only if $B$ is perfect as a $B^{\mathrm{op}} \otimes^{\mathbb{L}} B$-dg-module.

Proof. - (1) From [30, Lem. 7.5] we have a quasi-equivalence

$$
\widehat{\left(\widehat{T}_{\mathrm{pe}}\right)} \simeq \mathbb{R} \underline{H o m}\left(\widehat{T}_{\mathrm{pe}}^{\mathrm{op}}, \widehat{\mathbf{1}}\right) \rightarrow \widehat{T} \simeq \mathbb{R} \underline{H o m}\left(T^{\mathrm{op}}, \widehat{\mathbf{1}}\right) .
$$

Passing to the sub-dg-categories of perfect objects implies the result.

(2) For the property of having a compact generator, it follows by the same argument as above that

$$
\widehat{\left(\widehat{T}_{\mathrm{pe}}\right)} \simeq \widehat{T} .
$$

Suppose that $T$ is proper. Then, for any $x$ and $y$ in $T$, the complex

$$
T(x, y) \simeq \widehat{T}_{\mathrm{pe}}\left(\underline{h}_{x}, \underline{h}_{y}\right)
$$

is perfect. As $\left[\widehat{T}_{\mathrm{pe}}\right]$ is the thick closure of $[T]$, we see that this implies that for any $x$ and $y$ in $\widehat{T}_{\mathrm{pe}}$, the complex $\widehat{T}_{\mathrm{pe}}(x, y)$ is again perfect. This shows that $\widehat{T}_{\mathrm{pe}}$ is locally perfect and thus proper. Conversely, assume that $\widehat{T}_{\mathrm{pe}}$ is proper. As $T$ is quasi-equivalent to a full sub-dg-category of $\widehat{T}_{\mathrm{pe}}$ this implies that $T$ is locally perfect. Therefore $T$ is proper.

For a dg-category $T$, being smooth is equivalent to say that the Yoneda embedding $\underline{h}: T \rightarrow \widehat{T}$, considered as an object in $\mathbb{R} \underline{H o m}(T, \widehat{T}) \simeq \widehat{T^{\mathrm{op} \otimes^{\mathbb{L}}}} T$, is perfect. By [30, §7], we have

$$
\mathbb{R} \underline{\operatorname{Hom}}\left(\widehat{T}_{\mathrm{pe}}, \widehat{\left(\widehat{T}_{\mathrm{pe}}\right)}\right) \simeq \mathbb{R} \underline{\operatorname{Hom}}(T, \widehat{T}),
$$

easily implying that $T$ is smooth if and only if $\widehat{T}_{\text {pe }}$ is so.

(3) If $\widehat{T}$ is quasi-equivalent to $\widehat{B^{\circ p}}$, for some dg-algebra $B$, then $B$ as a dg-module over itself is a compact generator of $\left[\widehat{B^{\circ p}}\right] \simeq[\widehat{T}]$. Conversely, let $E$ be a compact generator of $[\widehat{T}]$, and we set $B=\widehat{T}(E, E)^{\mathrm{op}}$ to be the opposite dg-algebra of endomorphisms of $E$. The dg-algebra $B^{\mathrm{op}}$, considered as a dg-category with a unique object can be identified with the full sub-dg-category of $\widehat{T}_{\mathrm{pe}}$ consisting of the unique object $E$. Therefore, the restricted Yoneda embedding provides a morphism

$$
\widehat{T} \rightarrow \widehat{\left(\widehat{T}_{\mathrm{pe}}\right)} \rightarrow \widehat{B^{\mathrm{op}}}
$$

As $E$ is a compact generator, the induced functor

$$
\left.[\widehat{T}] \rightarrow \widehat{B^{\text {op }}}\right]
$$

is easily seen to be an equivalence (see for example [24]). This clearly implies that

$$
\widehat{T} \rightarrow \widehat{B^{\mathrm{op}}}
$$

is a quasi-equivalence. 
(4) Suppose that $T$ is a proper dg-category, then by (2) so is $\widehat{T}_{\mathrm{pe}}$. Therefore, if $\widehat{T} \simeq \widehat{B^{\mathrm{op}}}$, we find that $\widehat{T}_{\mathrm{pe}} \simeq \widehat{B_{\mathrm{pe}}^{\mathrm{op}}}$ is a proper dg-category. Therefore, $\widehat{B_{\mathrm{pe}}^{\mathrm{op}}}(B, B) \simeq B$ is a perfect complex of $k$-modules. Conversely, if $\widehat{T} \simeq \widehat{B^{\mathrm{op}}}$, with $B$ perfect as a complex of $k$-modules. The dg-category with a unique object $B$ is proper, and thus by (2) so is $\widehat{B_{\mathrm{pe}}^{\mathrm{op}}} \simeq \widehat{T}_{\mathrm{pe}}$. Again by (2) this implies that $T$ is proper.

(5) The proof is the same as for (4), and is a consequence of (2).

Let $T$ and $T^{\prime}$ be two dg-categories, then we can associate to any object $E$ in $\widehat{T \mathbb{Q L}^{\mathbb{L}} T^{\prime}}$ a morphism of dg-categories $F_{E}: T^{\mathrm{op}} \rightarrow \widehat{T^{\prime}}$ by letting for $x \in T$

$$
\begin{aligned}
F_{E}(x):\left(T^{\prime}\right)^{\mathrm{op}} & \rightarrow C(k) \\
y & \mapsto E(x, y) .
\end{aligned}
$$

This morphism will be considered as a morphism in $H o\left(d g-C a t_{\mathbb{V}}\right)$. By $[30, \S 7]$, there exists a natural isomorphism in $\mathrm{Ho}(\mathrm{dg}-\mathrm{Cat})$

$$
\widehat{T \otimes^{\mathbb{L}} T^{\prime}} \simeq \mathbb{R} \underline{H o m}\left(\left(T \otimes^{\mathbb{L}} T^{\prime}\right)^{\mathrm{op}}, \widehat{\mathbf{1}}\right) \simeq \mathbb{R} \underline{\operatorname{Hom}}\left(T^{\mathrm{op}}, \widehat{T^{\prime}}\right) .
$$

The morphism $F_{E}$ corresponds to $E$ through this identification.

Definition 2.7. - We say that an object $E \in \widehat{T \mathbb{Q ⿻}^{\mathbb{L}} T^{\prime}}$ is pseudo-perfect relatively to $T^{\prime}$ if the morphism $F_{E}$ factorizes, in $H o\left(d g-C a t_{\mathbb{V}}\right)$, through $\widehat{T_{\mathrm{pe}}^{\prime}}$ :

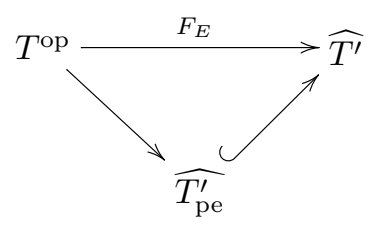

The full sub-dg-category of pseudo-perfect $\left(T \otimes^{\mathbb{L}} T\right)^{\mathrm{op}}$-dg-modules relatively to $T^{\prime}$ is denoted by

$$
T \widehat{\mathbb{Q}^{\mathbb{L}} T_{\mathrm{pspe}}^{\prime}} \subset \widehat{T \mathbb{Q L}^{\mathbb{L}} T^{\prime}}
$$

When $T^{\prime}=1$, we will simply use the terminology pseudo-perfect $T^{\mathrm{op}}$-dg-modules.

The main property of smooth and proper dg-categories is the following lemma, relating perfect and pseudo-perfect dg-modules.

LEMMA 2.8. - Let $T$ and $T^{\prime}$ be two dg-categories.

1. If $T$ is locally perfect, then a perfect object $E \in \widehat{T \otimes \mathbb{L}^{\prime}}$ is pseudo-perfect relative to $T^{\prime}$.

2. If $T$ is smooth, then a pseudo-perfect object $E \in T \otimes^{\mathbb{L}} T^{\prime}$ relative to $T^{\prime}$ is perfect.

3. If $T$ is smooth and locally perfect, then an object $E \in \widehat{T \otimes^{\mathbb{L}} T^{\prime}}$ is perfect if and only if it is pseudo-perfect relative to $T^{\prime}$.

Proof. - (1) As $\left[\widehat{T_{\mathrm{pe}}^{\prime}}\right]$ is the thick closure of $\left[T^{\prime}\right]$ in $\left[\widehat{T^{\prime}}\right]$, we see that it is enough to show that for any $(x, y) \in T \otimes^{\mathbb{L}} T^{\prime}$, the $\left(T \otimes^{\mathbb{L}} T^{\prime}\right)^{\mathrm{op}}$-dg-module $\underline{h}_{(x, y)}$ is pseudo-perfect with respect to $T^{\prime}$. But, for $z \in T$, we have

$$
\begin{aligned}
\underline{h}_{(x, y)}(z):\left(T^{\prime}\right)^{\mathrm{op}} & \rightarrow C(k) \\
t & \mapsto T(z, x) \otimes^{\mathbb{L}} T^{\prime}(t, y) .
\end{aligned}
$$

$4^{\mathrm{e}}$ SÉRIE - TOME $40-2007-\mathrm{N}^{\circ} 3$ 
In other words, the $\left(T^{\prime}\right)^{\text {op }}$-dg-module $\underline{h}_{(x, y)}(z)$ is of the form $T(z, x) \otimes^{\mathbb{L}} \underline{h}_{y}$. As $\underline{h}_{y}$ is perfect and $T(z, x)$ is a perfect complex by assumption on $T, \underline{h}_{(x, y)}(z)$ is a perfect $\left(T^{\prime}\right)^{\mathrm{op}}$-dg-module. In other words $\underline{h}_{(x, y)}$ is pseudo-perfect relative to $T^{\prime}$.

(2) Let us now assume that $T$ is smooth, and let $E$ be a pseudo-perfect $\left(T \otimes^{\mathbb{L}} T^{\prime}\right)^{\mathrm{op}}$-dg-module relative to $T^{\prime}$. We consider $F_{E}$ as an object in $\mathbb{R} \underline{H o m}\left(T^{\mathrm{op}}, \widehat{T^{\prime}}\right)$, as well as the composition morphism (well defined in $\mathrm{Ho}(d g$-Cat $)$ )

$$
\mathbb{R} \underline{\operatorname{Hom}}\left(T^{\mathrm{op}}, T^{\mathrm{op}}\right) \otimes^{\mathbb{L}} \mathbb{R} \underline{\operatorname{Hom}}\left(T^{\mathrm{op}}, \widehat{T^{\prime}}\right) \rightarrow \mathbb{R} \underline{\operatorname{Hom}}\left(T^{\mathrm{op}}, \widehat{T^{\prime}}\right) .
$$

Evaluating at $F_{E}$ provides a morphism

$$
\mathbb{R} \underline{\operatorname{Hom}}\left(T^{\mathrm{op}}, T^{\mathrm{op}}\right) \rightarrow \underline{\mathbb{H o m}}\left(T^{\mathrm{op}}, \widehat{T^{\prime}}\right)
$$

sending the identity to $F_{E}$. Using [30, §7], this last morphism can also be written as a continuous morphism (i.e. preserving arbitrary direct sums)

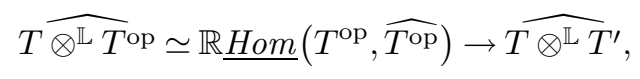

sending $T(-,-)$ to the object $E$. As $T$ is smooth, the object $T(-,-) \in\left[T{\widehat{\mathbb{Q L}^{\mathbb{L}}}}^{\mathrm{op}}\right]$ belongs to the smallest thick triangulated category containing the objects of the form $\underline{h}_{(x, y)}$ for some $(x, y) \in T \otimes^{\mathbb{L}} T^{\mathrm{op}}$. Therefore, it only remains to prove that the image of $\underline{h}_{(x, y)}$ is a perfect object in $\widehat{T \otimes^{\mathbb{L}} T^{\prime}}$. But, by construction this image is the $\left(T \otimes^{\mathbb{L}} T^{\prime}\right)^{\mathrm{op}}$-dg-module sending the object $(a, b) \in T \otimes^{\mathbb{L}} T^{\prime}$ to $T(a, x) \otimes^{\mathbb{L}} E(y, b)$. By assumption on $E$, the $\left(T^{\prime}\right)^{\text {op }}$-dg-module $E(y,-)$ is perfect. Therefore, the $\left(T \otimes^{\mathbb{L}} T^{\prime}\right)^{\mathrm{op}}$-dg-module $T(-, x) \otimes^{\mathbb{L}} E(y,-)$ being the external product of two perfect dg-modules is itself perfect.

(3) follows from (1) and (2).

COROLLARY 2.9.-

1. Let $T$ be a smooth and proper dg-category with a compact generator, and let $B$ be a dgalgebra with $\widehat{T} \simeq \widehat{B^{\mathrm{op}}}$. Then, an object $x \in \widehat{T}$ is perfect if and only if the corresponding $B$-dg-module is perfect as a complex of $k$-modules.

2. Let $T$ be a saturated dg-category. Then an object in $[\widehat{T}]$ is pseudo-perfect if and only if it is quasi-representable.

Proof. - This is the case $T^{\prime}=\mathbf{1}$ in Lemma 2.8 (3) and the definition of being saturated.

We will see in the next section that the moduli stack $\mathcal{M}_{T}$ does not classify exactly objects in $T$. However, by point (2) of the above corollary, this will be the case when $T$ is saturated. This is a dg-analog of our Remark 1.2.

Lemma 2.10. - Let $\left\{T_{\alpha}\right\}_{\alpha \in A}$ be a filtered diagram of objects in dg-Cat, with colimit $T$; then the natural morphism

$$
\operatorname{Colim}_{\alpha \in A}\left(\widehat{T_{\alpha}}\right)_{\mathrm{pe}} \rightarrow \widehat{T}_{\mathrm{pe}}
$$

is an isomorphism in $\mathrm{Ho}(\mathrm{dg}-\mathrm{Cat})$.

Proof. - Let $u_{\alpha}$ be the map from $T_{\alpha}$ in $T$,

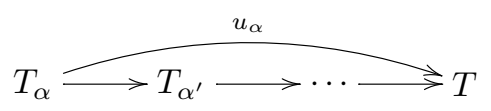


and let

$$
T_{\alpha}^{\mathrm{op}}-M o d \underset{u_{\alpha}^{*}}{\stackrel{u_{\alpha !}}{\gtrless}} T^{\mathrm{op}}-M o d
$$

be the Quillen adjunction defined by $u_{\alpha}$. This Quillen adjunction induces a morphism of dg-categories

$$
u_{\alpha !}: \widehat{T_{\alpha}} \rightarrow \widehat{T},
$$

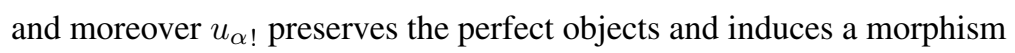

$$
u_{\alpha !}:\left(\widehat{T_{\alpha}}\right)_{\mathrm{pe}} \rightarrow \widehat{T}_{\mathrm{pe}} .
$$

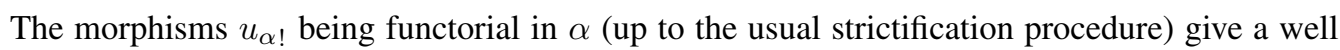
defined morphism of dg-categories

$$
\phi: \operatorname{Colim}_{\alpha \in A}\left(\widehat{T}_{\alpha}\right)_{\mathrm{pe}} \rightarrow \widehat{T}_{\mathrm{pe}} .
$$

Let us start to prove that the morphism $\phi$ is quasi-fully faithful. For this, we set $T_{0}:=$ $\operatorname{Colim}_{\alpha} \widehat{T_{\alpha}}$. Let $E$ and $F$ be two objects in $T_{0}$, and we choose $\alpha_{0} \in A$, and two objects $E_{\alpha_{0}}$, $F_{\alpha_{o}} \in\left(\widehat{T_{\alpha_{o}}}\right)_{\text {pe }}$ representing $E$ and $F$. By definition we have

$$
T_{0}(E, F)=\operatorname{Colim}_{\alpha \in \alpha_{0} / A}\left(\widehat{T_{\alpha}}\right)\left(i_{\alpha !}\left(E_{\alpha_{o}}\right), i_{\alpha !}\left(F_{\alpha_{o}}\right)\right),
$$

where $\left(i_{\alpha !}, i_{\alpha}^{*}\right)$ is the Quillen adjunction defined by the morphism $i_{\alpha}: T_{\alpha_{o}} \rightarrow T_{\alpha}$, for $\alpha \in \alpha_{o} / A$. On the other hand

$$
\widehat{T}(\phi(E), \phi(F))=\widehat{T}\left(u_{\alpha_{o} !}\left(E_{\alpha_{o}}\right), u_{\alpha_{o} !}\left(F_{\alpha_{o}}\right)\right) .
$$

By adjunction, as $E_{\alpha_{o}}$ is a perfect object, we have

$$
T_{0}(E, F) \simeq \underline{H o m}_{T_{\alpha_{0}}^{\mathrm{op}}}\left(E_{\alpha_{o}}, \operatorname{Colim}_{\alpha \in \alpha_{0} / A} i_{\alpha}^{*} i_{\alpha !}\left(F_{\alpha_{o}}\right)\right)
$$

and

$$
\widehat{T}(\phi(E), \phi(F)) \simeq \underline{H o m}_{T_{\alpha_{0}}^{\mathrm{op}}}\left(E_{\alpha_{o}}, u_{\alpha_{o}}^{*} u_{\alpha_{o} !}\left(F_{\alpha_{o}}\right)\right),
$$

where $\underline{H o m}_{T_{\alpha_{0}}^{\mathrm{op}}}$ denotes the $C(k)$-enriched Hom's of the model category $T_{\alpha_{0}}^{\mathrm{op}}-M o d$. Therefore, it is enough to show that the natural morphism

$$
\beta: \operatorname{Colim}_{\alpha \in \alpha_{0} / A} i_{\alpha}^{*} i_{\alpha !}\left(F_{\alpha_{o}}\right) \rightarrow u_{\alpha_{o}}^{*} u_{\alpha_{o} !}\left(F_{\alpha_{o}}\right)
$$

is an equivalence in $T_{\alpha_{o}}^{\mathrm{op}}-$ Mod.

The two morphisms $\operatorname{Colim}_{\alpha} i_{\alpha}^{*} i_{\alpha \text { ! }}$ and $u_{\alpha_{o}}^{*} u_{\alpha_{o} !}$, from $\left[\widehat{T_{\alpha_{0}}}\right]$ to itself, are triangulated and preserve direct sums. Furthermore, there exists a natural transformation $\beta$ between them, which is easily seen to induce isomorphisms when evaluated at any representable objects $\underline{h}_{x} \in\left[\widehat{T_{\alpha_{0}}}\right]$. As the representable objects are compact generators, this implies that $\beta$ is an isomorphism. In particular, evaluated at the object $F_{\alpha_{0}}$, we get that the natural morphism

$$
\operatorname{Colim}_{\alpha} i_{\alpha}^{*} i_{\alpha !}\left(F_{\alpha_{o}}\right) \rightarrow u_{\alpha_{o}}^{*} u_{\alpha_{o} !}\left(F_{\alpha_{o}}\right)
$$

$4^{\text {e }}$ SÉRIE - TOME $40-2007-\mathrm{N}^{\circ} 3$ 
is an equivalence.

We have proved that $\phi$ is fully faithful, and it remains to show that $\phi$ is also quasi-essentially surjective. Let $E$ be a perfect object in $\widehat{T}$, then $E$ is equivalent to a retract of an object $F$ which is obtained as the finite colimit of push-out square

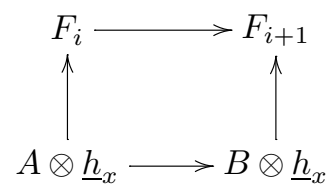

for some $x \in T$, and some cofibration $A \rightarrow B$ in $C(k)$ with $A$ and $B$ bounded complexes of projective modules of finite type (because of Proposition 2.2 (4)).

As $\phi$ is defined by $\phi\left(\left(E_{\alpha}\right)\right)=u_{\alpha !}\left(E_{\alpha}\right)$ for some $\alpha$ and is quasi-fully faithful, and as $u_{\alpha !}$ is a left Quillen functor which preserves homotopy push-out and tensor product by $A \in C(k)$, it is enough to show that any representable module $\underline{h}^{x}$ in $[\widehat{T}]$ is in the image of $[\phi]$. But, this is a consequence of the fact that $u_{\alpha !}\left(\underline{h}_{x_{\alpha}}\right) \simeq \underline{h}_{u_{\alpha}\left(x_{\alpha}\right)}$.

An important consequence of Lemma 2.10 is the following finiteness statement.

LEMMA 2.11. - Let B be a dg-algebra, then B is homotopically finitely presented in $d g$-Alg if and only iffor any filtered diagram $\left\{T_{\alpha}\right\}_{\alpha \in A}$ in $d g$-Cat, with colimit $T$, the natural morphism

$$
\operatorname{Colim}_{\alpha \in A} \operatorname{Map}_{d g-C a t}\left(B,\left(\widehat{T_{\alpha}}\right)_{\mathrm{pe}}\right) \rightarrow \operatorname{Map}_{d g-C a t}\left(B,(\widehat{T})_{\mathrm{pe}}\right)
$$

is an equivalence.

Proof. - The functor which associates to a dg-algebra $B$ the dg-category with one object defines a Quillen adjunction

$$
d g-A l g \underset{E}{\rightleftarrows} d g-C a t_{*}
$$

between the model category of dg-algebras and the model category of pointed dg-categories $d g-C a t_{*}:=1 / d g$-Cat. The right adjoint $E$ is defined by sending a pointed dg-category $T$ to the dg-algebra $T(t, t)$ of endomorphisms of the distinguished object $t$ of $T$. Then, for any dg-algebra $B$ and any pointed dg-category $T$ there is an equivalence $\operatorname{Map}_{d g-A l g}(B, E(T)) \simeq$ $\operatorname{Map}_{d g-\mathrm{Cat}_{*}}(B, T)$.

Let $B$ be a dg-algebra, then the unit of $B$ defines a morphism $1 \rightarrow B$ in $d g$-Cat, and for any dg-category $D$ we obtain a homotopy fibration

$$
\operatorname{Map}_{d g-C a t_{*}}(B, D) \rightarrow M_{d g-C a t}(B, D) \rightarrow M_{d g-C a t}(\mathbf{1}, D)
$$

where $D$ is pointed via the base point $u: \mathbf{1} \rightarrow D$. By adjunction, we find a homotopy fibration

$$
\operatorname{Map}_{d g-A l g}(B, E(D)) \rightarrow M_{d g-C a t}(B, D) \rightarrow \operatorname{Map}_{d g-C a t}(\mathbf{1}, D)
$$


Let $D_{\alpha}$ be the dg-category $\left(\widehat{T_{\alpha}}\right)_{\text {pe }}$, then by Lemma 2.10 we have $\operatorname{Colim}_{\alpha} D_{\alpha}$ equivalent to $D=\widehat{T}_{\mathrm{pe}}$ and $\operatorname{Colim}_{\alpha} E\left(D_{\alpha}\right)$ equivalent to $E(D)$, and we get the commutative diagram

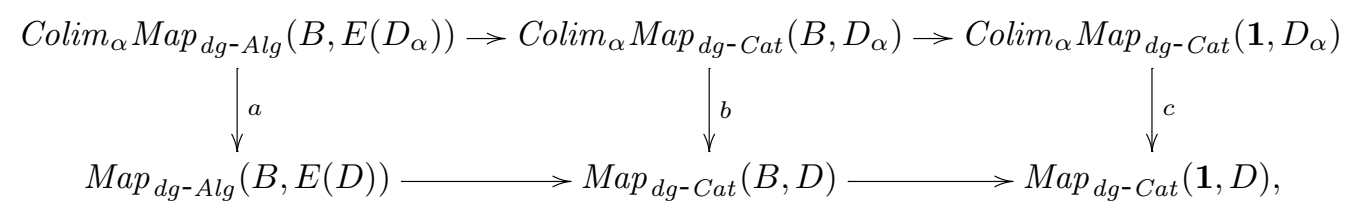

in which each horizontal row is a fibration sequence, and as $\mathbf{1}$ is homotopically finitely presented in $d g$-Cat, the vertical arrow $c$ is an equivalence.

If $B$ is homotopically finitely presented in $d g$ - $A l g$, the vertical arrow $a$ is also an equivalence, then $b$ is an equivalence too. Conversely we consider the same diagram with $T_{\alpha}$ the dg-category associated to a dg-algebra $C_{\alpha}$, then $E\left(T_{\alpha}\right)=C_{\alpha}$. Then if the vertical arrow $b$ is an equivalence we deduce that $B$ is homotopically finitely presented in $d g-A l g$.

COROLLARY 2.12. - Let $T$ be a dg-category with a compact generator; then $T$ is of finite type if and only if any dg-algebra $B$ such that $\widehat{T}$ is quasi-equivalent to $\widehat{B^{\mathrm{OP}}}$, is homotopically finitely presented in $\mathrm{dg}$-Alg.

Proof. - We have to show that if $B$ and $C$ are two dg-algebras such that the dg-categories $\widehat{B^{\text {op }}}$ and $\widehat{C^{\mathrm{op}}}$ are equivalent, and if $C$ is homotopically finitely presented in $d g-A l g$ then $B$ is also homotopically finitely presented. This a consequence of Lemma 2.11 and of the main result of $[30, \S 7]$.

COROLLARY 2.13. - A smooth and proper dg-category $T$ is of finite type.

Proof. - By Lemma 2.11 and the main result of [30, §7] it is enough to show that for any filtered diagram $\left\{D_{\alpha}\right\}_{\alpha \in A}$ in $d g$-Cat, the natural map

$$
\operatorname{Colim}_{\alpha \in A} \operatorname{Map}_{d g-C a t}\left(T^{\mathrm{op}},\left(\widehat{D_{\alpha}}\right)_{\mathrm{pe}}\right) \rightarrow \operatorname{Map}_{d g-C a t}\left(T^{\mathrm{op}},(\widehat{D})_{\mathrm{pe}}\right)
$$

is an equivalence.

By definition and by results of $[30, \S 7]$, for any dg-categories $T$ and $T^{\prime}$ there exists a natural equivalence of simplicial sets

$$
\operatorname{Map}\left(T^{\mathrm{op}}, \widehat{D}_{\mathrm{pe}}\right) \simeq \operatorname{Map}\left(\mathbf{1},\left(\widehat{T \otimes^{\mathbb{L}} D}\right)_{\mathrm{pspe}}\right) .
$$

Moreover, if $T$ is smooth and proper we deduce from Lemma 2.8 the equivalence

$$
\operatorname{Map}\left(T^{\mathrm{op}}, \widehat{D}_{\mathrm{pe}}\right) \simeq \operatorname{Map}\left(\mathbf{1},\left(\widehat{T \otimes^{\mathbb{L}} D}\right)_{\mathrm{pe}}\right) .
$$

Let $D_{\alpha}$ be a filtered diagram in $d g$-Cat with colimit $D$; then we have

$$
T \otimes^{\mathbb{L}} D \simeq T \otimes^{\mathbb{L}}\left(\operatorname{Colim}_{\alpha} D_{\alpha}\right) \simeq \operatorname{Colim}_{\alpha}\left(T \otimes^{\mathbb{L}} D_{\alpha}\right),
$$

and by Lemma 2.10

$$
\left(\widehat{T \otimes^{\mathbb{L}} D}\right)_{\mathrm{pe}} \simeq \operatorname{Colim}_{\alpha}\left(\left(\widehat{T \otimes^{\mathbb{L}} D}\right)_{\mathrm{pe}}\right) .
$$

Next, as $\mathbf{1}$ is homotopically finitely presented in $d g$-Cat, we have

$4^{\mathrm{e}}$ SÉRIE - TOME $40-2007-\mathrm{N}^{\circ} 3$ 


$$
\begin{aligned}
\operatorname{Map}\left(T^{\mathrm{op}}, \widehat{D}_{\mathrm{pe}}\right) & \simeq \operatorname{Map}\left(\mathbf{1}, \operatorname{Colim}_{\alpha}\left(\left(\widehat{T \mathbb{Q ⿻}^{\mathbb{L}} D_{\alpha}}\right)_{\mathrm{pe}}\right)\right) \\
& \simeq \operatorname{Colim}_{\alpha} \operatorname{Map}\left(\mathbf{1},\left(\widehat{T \widehat{\otimes L}^{\mathbb{L}} D_{\alpha}}\right)_{\mathrm{pe}}\right) \simeq \operatorname{Colim}_{\alpha} \operatorname{Map}\left(T^{\mathrm{op}},\left(\widehat{D}_{\alpha}\right)_{\mathrm{pe}}\right) .
\end{aligned}
$$

Corollary 2.13 provides a very useful tool to construct dg-categories of finite type, as in practice it is often more easy to check that a dg-category is saturated than to check directly that it is of finite type.

Corollary 2.13 has the following converse.

PROPOSITION 2.14. - Any dg-category of finite type is smooth.

Proof. - It is enough to show that for any homotopically finitely presented dg-algebra $B, B$ is homotopically finitely presented as a $B \otimes^{\mathbb{L}} B^{\mathrm{op}}$-dg-module. For this, we consider the exact triangle of $B \otimes^{\mathbb{L}} B^{\mathrm{op}}$-dg-modules

$$
I_{B} \longrightarrow B \otimes^{\mathbb{L}} B^{\mathrm{op}} \longrightarrow B
$$

where the morphism of the right-hand side is given by multiplication in $B$. This triangle shows that it is enough to show that $I_{B}$ is homotopically finitely presented as a $B \otimes^{\mathbb{L}} B^{\mathrm{op}}$-dg-module. But, for any $B \otimes^{\mathbb{L}} B^{\text {op }}$-dg-module $M$ we have

$$
M a p_{B \otimes^{\mathbb{L}} B^{\mathrm{op}-M o d}}\left(I_{B}, M\right) \simeq M a p_{d g-A l g / B}(B, B \oplus M),
$$

where $B \oplus M$ is the trivial square zero extension of $B$ by $M$ (see [18]). Therefore, the fact that $I_{B}$ is homotopically finitely presented follows from the fact that $B$ is homotopically finitely presented as a dg-algebra.

\subsection{Geometric $D^{-}$-stacks}

We will use the theory of $D^{-}$-stacks, as presented in [35, §2.2], from which we recall the basic notations.

We let $s k$ - $C A l g$ be the category of simplicial commutative (associative and unital) $k$-algebras. By definition $k-D^{-} A f f$ is the opposite category of $s k-C A l g$. The category $s k-C A l g$ is endowed with a model structure for which fibrations and equivalences are defined on the underlying simplicial sets. The category $k-D^{-} A f f$ is endowed with the opposite model structure. For any $A \in s k$-CAlg, we denote by $A$-Mods the category of simplicial modules over the simplicial ring $A$. It is endowed with its natural model structure for which fibrations and equivalences are defined on the underlying simplicial sets.

We define natural extensions of the notions of étale, smooth and flat morphisms to the case of simplicial algebras as follows. A morphism $A \rightarrow B$ in $s k$-CAlg is called étale (resp. smooth, resp. flat) if

- the induced morphism $\pi_{0}(A) \rightarrow \pi_{0}(B)$ is an étale (resp. smooth, resp. flat) morphism of commutative rings,

- for any $i$, the induced morphism

$$
\pi_{i}(A) \otimes_{\pi_{0}(A)} \pi_{0}(B) \rightarrow \pi_{i}(B)
$$

is an isomorphism.

A morphism $A \rightarrow B$ will be called an étale covering if it is étale and if furthermore the morphism of affine schemes $\operatorname{Spec} \pi_{0}(B) \rightarrow \operatorname{Spec} \pi_{0}(A)$ is surjective. This notion endows the model category $k-D^{-} A f f$ with a structure of a ( $\mathbb{V}$-small $)$ model site, and from the general theory 
of [34] we can construct a corresponding model category of stacks $k-D^{-} A f f^{\sim, \text { et }}$. The underlying category of $k-D^{-} A f f^{\sim \text {,et }}$ is the category of functors $s k-C A l g \rightarrow S S e t_{\mathbb{V}}$, from commutative simplicial $k$-algebras to $\mathbb{V}$-small simplicial sets. The model structure on $k$ - $D^{-} A f f^{\sim, \text { et }}$ is a certain left Bousfield localization of the projective levelwise model category structure on simplicial presheaves, with respect to equivalences of simplicial rings and nerves of étale hyper coverings.

By definition, the homotopy category of $D^{-}$-stacks is $H o\left(k-D^{-} A f f^{\sim, \text { et }}\right)$, and is denoted as in [35] by $D^{-} S t(k)$. In the same way, for any object $F \in D^{-} S t(k)$, we will set

$$
D^{-} S t(F):=H o\left(k-D^{-} A f f^{\sim, e t} / R F\right),
$$

where $R F$ is a fibrant replacement of $F$ (note that $D^{-} S t(F)$ is not equivalent to the $\left.D^{-} S t(k) / F\right)$.

For any $A \in s k-C A l g$, we define its spectrum $\operatorname{Spec} A \in D^{-} S t(k)$ by the formula

$$
\begin{aligned}
\underline{\text { Spec A } A k-C A l g} & \rightarrow \text { SSet } \\
B & \mapsto \underline{H o m}(A, B),
\end{aligned}
$$

where $\operatorname{Hom}(A, B)$ are the natural simplicial Hom's of the category sk-CAlg. The functor $A \mapsto \underline{\operatorname{Hom}}(A, B)$ is almost a right Quillen functor ${ }^{3}$

$$
\underline{\text { Spec }}: s k-C A l g^{\mathrm{op}} \rightarrow k-D^{-} A f f^{\sim, \mathrm{et}},
$$

and its right derived functor

$$
\mathbb{R} \underline{S p e c}: H o(s k-C A l g)^{\mathrm{op}}=H o\left(k-D^{-} A f f\right) \rightarrow D^{-} S t(k)
$$

is fully faithful. The $D^{-}$-stacks in the essential image of the functor $\mathbb{R} S p e c$ are by definition the representable $D^{-}$-stacks. The notion of étale, smooth and flat morphisms between simplicial $k$-algebras extend in a unique way to morphisms between representable $D^{-}$-stacks. A $D^{-}$-stack is then called $(-1)$-geometric if it is isomorphic in $D^{-} S t(k)$ to a representable $D^{-}$-stack.

For an integer $n \geqslant 0$, an object $F \in D^{-} S t(k)$ is an n-geometric $D^{-}$-stack if it satisfies the following two conditions.

- The morphism $F \rightarrow F \times{ }^{h} F$ is $(n-1)$-representable.

- There exist a family of representable $D^{-}$-stacks $\left\{X_{i}\right\}$ and a covering

$$
\coprod_{i} X_{i} \rightarrow F
$$

such that each morphism $X_{i} \rightarrow F$ is smooth. Such a family $\left\{X_{i}\right\}$ together with the morphism $\coprod_{i} X_{i} \rightarrow F$ is called an $n$-atlas for $F$.

In order for the previous notion to make sense we need to finish the induction on $n$ by setting the following.

- A morphism $f: F \rightarrow G$ between $D^{-}$-stacks is $n$-representable if for any representable $X$ and any morphism $X \rightarrow G$, the $D^{-}$-stack $F \times{ }_{G}^{h} X$ is $n$-geometric.

- An $n$-representable morphism $f: F \rightarrow G$ is smooth, if for any representable $X$ and any morphism $X \rightarrow G$, there exists an $n$-atlas $\left\{Y_{i}\right\}$ of the $D^{-}$-stack $F \times{ }_{G}^{h} X$, such that each morphism $Y_{i} \rightarrow X$ is a smooth morphism between representable $D^{-}$-stacks.

\footnotetext{
${ }^{3}$ This functor is not right Quillen because it does not possess a left adjoint (because of universes issues). However, it does preserve fibrations and trivial fibrations and thus we can define a total right derived functor.

4e SÉRIE - TOME $40-2007-\mathrm{N}^{\circ} 3$
} 
We will also use the following terminology.

- A $D^{-}$-stack $F$ is quasi-compact if there exist a representable $D^{-}$-stack $X$ and a covering $X \rightarrow F$.

- A morphism of $D^{-}$-stacks $f: F \rightarrow G$ is quasi-compact if for any representable $X$ and any morphism $X \rightarrow G$, the $D^{-}$-stack $F \times{ }_{G}^{h} X$ is quasi-compact.

- By induction on $n$, an $n$-geometric $D^{-}$-stack $F$ is strongly quasi-compact if it is quasicompact, and if for any two representable $X$ and $Y$ and any morphisms $X \rightarrow F, Y \rightarrow F$, the $(n-1)$-geometric $D^{-}$-stack $X \times{ }_{F}^{h} Y$ is strongly quasi-compact.

- An $n$-representable morphism $F \rightarrow G$ is strongly quasi-compact if for any representable $D^{-}$-stack $X$, and any morphism $X \rightarrow F, F \times{ }_{G}^{h} X$ is a strongly quasi-compact $n$-geometric $D^{-}$-stack.

- A representable $D^{-}$-stack $F \simeq \mathbb{R}$ Spec $A$ is finitely presented if for any filtered system of objects $B_{i}$ in $s k$-CAlg, the morphism

$$
\operatorname{Colim}_{i} \operatorname{Map}\left(A, B_{i}\right) \rightarrow \operatorname{Map}\left(A, \operatorname{Colim}_{i} B_{i}\right)
$$

is an equivalence.

- An $n$-geometric $D^{-}$-stack $F$ is locally of finite presentation if it has an $n$-atlas $\left\{X_{i}\right\}$ such that each $X_{i}$ is finitely presented.

- An $n$-geometric $D^{-}$-stack $F$ is strongly of finite presentation if it is locally of finite presentation and strongly quasi-compact.

LEMMA 2.15. - The n-geometric $D^{-}$-stacks (resp. $n$-geometric $D^{-}$-stacks locally of finite presentation, resp. $n$-geometric $D^{-}$-stacks strongly of finite presentation) are stable by homotopy pull-backs and retracts.

Proof. - The stability by homotopy pull-backs is easily reduced by induction on $n$ to the case of representable $D^{-}$-stacks, for which the proof is straightforward (see e.g. [35, Prop. 1.3.3.3]). The stability by retracts requires a separate treatment.

Let $F$ be an $n$-geometric $D^{-}$-stack, and let $F_{0}$ be a retract in $D^{-} S t(k)$ of $F$. Up to equivalences we can arrange things so that there exists a diagram of fibrant objects in $k-D^{-} A f f^{\sim, \text { et }}$

$$
F_{0} \stackrel{i}{\longrightarrow} F \stackrel{r}{\longrightarrow} F_{0}
$$

such that $r \circ i=$ id (i.e. that $F_{0}$ is a retract of $F$ as a simplicial presheaf). Let $p=i \circ r$ be the corresponding projector on $F$. As $p^{2}=p$, this defines an action of the monoid $M$ freely generated by a projector on $F$. We then have an isomorphism in $D^{-} S t(k)$

$$
F_{0} \simeq \lim _{B M} F \simeq \operatorname{Holim}_{B M} F
$$

where $F$ is considered as a diagram over $B M$, the category with one object and $M$ as its endomorphisms. The homotopy limit on the right hand side can also be described as a homotopy limit of a cosimplicial diagram $G_{*}$ whose object in degree $k$ is $G_{k}:=\operatorname{Hom}\left(M^{k}, F\right)$ and with transitions maps defined using the action of $M$ on $F$ and the multiplication in $M$. The fact that $F_{0}$ is $n$-geometric now follows from the following general result.

SUB-LEMMA 2.16. - Let $G_{*}: \Delta \rightarrow k-D^{-}$Aff ${ }^{\sim \text { et }}$ be a cosimplicial diagram such that $G_{k}$ is $n$-geometric for any $k$. Then, Holim ${ }_{\Delta} G_{*}$ is also $n$-geometric. 
Proof of the Sub-lemma 2.16. - Let $\Delta^{*}$ be the cosimplicial object in $k-D^{-} A f f^{\sim, \text { et }}$ sending $k$ to the constant simplicial presheaf $\Delta^{k}$. We have

$$
\operatorname{Holim}_{\Delta} G_{*} \simeq \operatorname{Map}\left(\Delta^{*}, G_{*}\right)
$$

where the mapping spaces on the right are considered in the model category of cosimplicial simplicial sets (e.g. with the Reedy model structure of $[9, \S 5.2])$. In other words, for two cosimplicial $D^{-}$-stacks $X_{*}$ and $Y_{*}$ we denote by $\operatorname{Map}\left(X_{*}, Y_{*}\right)$ the $D^{-}$-stack sending $A \in \operatorname{sk}-C A l g$ to $\operatorname{Map}_{S \operatorname{Set}} \Delta\left(X_{*}(A), Y_{*}(A)\right)$.

We consider the subsimplicial presheaf $\Delta_{m}^{*}$ of $\Delta^{*}$ sending $k$ to the $m$-th skeleton $S q_{m} \Delta^{k}$ of $\Delta^{k}$. We clearly have

$$
\Delta^{*} \simeq \operatorname{Colim}_{m} \Delta_{m}^{*}
$$

and thus

$$
\operatorname{Holim}_{\Delta} G_{*} \simeq \operatorname{Map}\left(\Delta^{*}, G_{*}\right) \simeq \operatorname{Holim}_{m} \operatorname{Map}\left(\Delta_{m}^{*}, G_{*}\right) .
$$

Moreover, for any $m$ we have a homotopy co-Cartesian square of cosimplicial objects

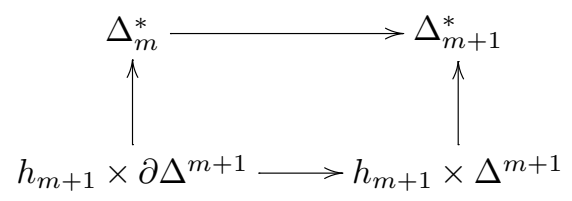

where $h_{m+1}$ is the cosimplicial object sending $k$ to $\Delta^{k}(m+1)=H o m([m+1],[k])$. Therefore, there exists a homotopy Cartesian square of $D^{-}$-stacks

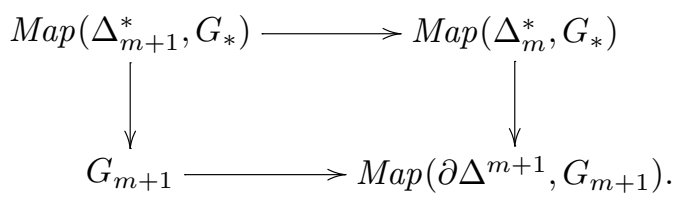

From this description we deduce by induction on $m$ that each $\operatorname{Map}\left(\Delta_{m}^{*}, G_{*}\right)$ is an $n$-geometric $D^{-}$-stack. Moreover, we also see by induction on $m$ that the morphism

$$
\operatorname{Map}\left(\Delta_{m+1}^{*}, G_{*}\right) \rightarrow \operatorname{Map}\left(\Delta_{m}^{*}, G_{*}\right)
$$

is $(n-m-1)$-representable, and thus is $(-1)$-representable as soon as $m \geqslant n$ (the case $m=0$ is true because the diagonal $G_{1} \rightarrow G_{1} \times{ }^{h} G_{1}$ is $(n-1)$-geometric by definition). Using the fact that the $(-1)$-representable $D^{-}$-stacks are stable by homotopy limits we deduce from this that the morphism

$$
\operatorname{Holim}_{\Delta} G_{*} \rightarrow \operatorname{Map}\left(\Delta_{m}^{*}, G_{*}\right)
$$

is $(-1)$-representable as soon as $m \geqslant n$. This implies that $\operatorname{Holim}_{\Delta} G_{*}$ is $n$-geometric.

By the previous sub-lemma we know that $F_{0}$ is $n$-geometric. If moreover $F$ is quasi-compact, the fact that the morphism $r: F \rightarrow F_{0}$ is a covering implies that $F_{0}$ is also quasi-compact. By induction on $n$ we also see that $F_{0}$ is strongly quasi-compact if $F$ is so. To finish the proof of the lemma it only remains to show that $F_{0}$ is locally of finite presentation if $F$ is so. We keep the

$4^{\text {e }}$ SÉRIE - TOME $40-2007-\mathrm{N}^{\circ} 3$ 
same notations as in Sub-lemma 2.16. We first consider the truncated stacks $t_{0}\left(F_{0}\right)$ and $t_{0}(F)$ (see below or [35, §2.2.4]). The stack $t_{0}\left(F_{0}\right)$ is now a retract of $t_{0}(F)$, and we can use the same description of $t_{0}\left(F_{0}\right)$ as the homotopy limit of $t_{0}\left(G_{*}\right)$ as before. But now, the stacks $t_{0}\left(F_{0}\right)$ and $t_{0}(F)$ being $n$-geometric are also $n$-truncated, and thus we see that for $m>n$ the natural map

$$
\operatorname{Holim}_{\Delta} t_{0}\left(G_{*}\right) \rightarrow \operatorname{Map}\left(\Delta_{m}^{*}, t_{0}\left(G_{*}\right)\right)
$$

is an isomorphism in the homotopy category of stacks. By induction on $m$ we see that $t_{0}\left(F_{0}\right)$ is thus locally of finite presentation as a stack. Finally, for any $A \in s k-C A l g$, with $X:=\mathbb{R} \operatorname{Spec} A$, and any morphism $u: X \rightarrow F_{0}$, the cotangent complex $\mathbb{L}_{u}$ is a retract of the cotangent complex $\mathbb{L}_{i \circ u}$ (see [35] for the definition of cotangent complexes). As $F$ is locally of finite presentation the cotangent complex $\mathbb{L}_{i \circ u}$ is perfect, and thus so is $\mathbb{L}_{u}$. The $D^{-}$-stack $F_{0}$ is therefore $n$-geometric and such that the truncation $t_{0}\left(F_{0}\right)$ is locally of finite presentation, and its cotangent complexes are perfect. By [35, Prop. 2.2.2.4] this implies that $F_{0}$ is locally of finite presentation.

We finish by the following extended definition of geometric stacks.

DEFINITION 2.17. - A $D^{-}$-stack $F$ is called locally geometric if it can be written as a filtered colimit

$$
F \simeq \operatorname{Hocolim}_{i} F_{i}
$$

such that the following conditions are satisfied.

1. Each $D^{-}$-stack $F_{i}$ is $n$-geometric for some $n$ (depending on $i$ ).

2. Each morphism $F_{i} \rightarrow F$ is a monomorphism (recall this means that the natural morphism $F_{i} \rightarrow F_{i} \times{ }_{F}^{h} F_{i}$ is an isomorphism in $D^{-} S t(k)$, or equivalently that the morphism induces an isomorphism between $F_{i}$ and a full sub- $D^{-}$-stack of $F$ ).

A locally geometric $D^{-}$-stack $F$ is locally of finite presentation if each of $F_{i}$ as above can be chosen to be locally of finite presentation.

Note that in the definition above, each morphism $F_{i} \rightarrow F$ is a monomorphism and thus so are the transition morphisms $F_{i} \rightarrow F_{j}$. In particular, the morphisms $F_{i} \rightarrow F_{j}$ are always formally étale. If furthermore $F$ is locally of finite presentation, so that each $F_{i}$ can itself be chosen locally of finite presentation, then the morphisms $F_{i} \rightarrow F_{j}$ will be a formally étale monomorphism locally of finite presentation, and thus will be a Zariski open immersion (see [35, Def. 2.2.3.5]). In other words, a locally geometric $D^{-}$-stack which is locally of finite presentation is always the colimit of a filtered system of Zariski open immersions between $n$-geometric $D^{-}$-stacks locally of finite presentation.

LEMMA 2.18. - Let $F$ be a locally geometric $D^{-}$-stack. Then, $F$ is n-geometric if and only if its diagonal is $(n-1)$-representable.

Proof. - Indeed, by definition it is enough to show that $F$ is a smooth $n$-atlas. For this, we write $F$ as a union of $F_{i}$ as in Definition 2.17. As each $F_{i}$ is a full sub- $D^{-}$-stack of $F$ its diagonal is again $(n-1)$-representable. Therefore, each $F_{i}$ is in fact $n$-geometric. We let $\left\{U_{i, j}\right\}_{j}$ be a smooth $n$-atlas of $F_{i}$, then clearly the $\left\{U_{i, j}\right\}_{i, j}$ is a smooth $n$-atlas for $F$.

To finish this part, we recall from [35, §2.1] the existence of the homotopy category $S t(k)$ of stacks over $k$, being defined as the homotopy category of the model category of simplicial presheaves on the site of affine $k$-schemes endowed with the étale topology. Its objects can be described as functors

$$
F: k-C A l g \rightarrow S S e t,
$$


from the category $k$-CAlg of commutative $k$-algebras to the category of simplicial sets, satisfying the descent condition for étale hypercoverings of affine $k$-schemes. There exists a natural embedding $k$-CAlg $\rightarrow s k$ - $C A l g$ which consists of considering a commutative $k$-algebra as a constant simplicial commutative $k$-algebra. This embedding induces a restriction functor, also called the truncation functor

$$
t_{0}: D^{-} S t(k) \rightarrow S t(k)
$$

The functor $t_{0}$ has a left adjoint

$$
i: S t(k) \rightarrow D^{-} S t(k)
$$

which is fully faithful. Therefore, the category $S t(k)$ will be considered as a full sub-category of $D^{-} S t(k)$. However, we warn the reader that the functor $i$ does not preserve homotopy fiber products except along flat morphisms.

As for $D^{-}$-stacks, there exists a notion of $n$-geometric stack obtained by using similar definitions (see $[35, \S 2.1]$ for details). The inclusion functor is moreover compatible with these definitions in the sense that a stack $F$ is $n$-geometric if and only if the $D^{-}$-stack $i(F)$ is so. By definition, a stack $F$ is locally geometric if the $D^{-}$-stack $i(F)$ is so. Be careful that $i$ does not preserve monomorphisms, so Definition 2.17 must be translated carefully in the language of stacks. In any case, a stack is locally geometric and locally finitely presented if and only if it can be written as a union of open substacks which are $n$-geometric and locally of finite presentation.

By definition, an Artin $n$-stack is a stack which is $n$-truncated (i.e. for any $A \in k$-CAlg the simplicial set $F(A)$ is n-truncated) and $m$-geometric for some integer $m \geqslant 0$ (see [35, 2.1]). Finally, we mention the following two results.

LEMMA 2.19. - Let $F$ be a locally geometric stack which is $n$-truncated for some $n \geqslant 0$. Then $F$ is $(n+1)$-geometric, and in particular is an Artin $n$-stack.

Proof. - This follows easily by induction on $n$. For $n=0$ this is [35, Rem. 2.1.1.5].

Lemma 2.20. - Let $F \rightarrow G$ be an n-representable morphism of $D^{-}$-stacks. If $G$ is $n$-geometric then so is $F$.

Proof. - This follows from stability of $n$-representable morphisms by composition (see [35, Prop. 1.3.3.3]) and from the local character of being $n$-geometric (see [35, Prop. 1.3.3.4]).

\subsection{Tor amplitude of perfect dg-modules}

Let us fix $A \in s k$-CAlg. We will denote by $N(A)$ the normalized cochain complex of $k$-modules associated to $A$. Recall that the shuffle products produce for two simplicial $k$-modules $X$ and $Y$, a natural morphism of complexes

$$
N(X) \otimes N(Y) \rightarrow N(X \otimes Y)
$$

which is furthermore associative, unital and commutative. This can be used to define a natural structure of a commutative differential graded $k$-algebra on $N(A)$. This construction provides a functor

$$
N: s k-C A l g \rightarrow k-c d g a,
$$

which sends equivalences of simplicial algebras to quasi-isomorphisms of dg-algebras. Furthermore, for $A \in s k-C A l g$, the normalization provides a functor

$$
A-\operatorname{Mod}_{s} \rightarrow N(A)-M o d,
$$

$4^{\text {e }}$ SÉRIE - TOME $40-2007-\mathrm{N}^{\circ} 3$ 
from the category of simplicial $A$-modules to the category of $N(A)$-dg-modules. This last functor also sends equivalences of simplicial $A$-modules to quasi-isomorphisms. It even induces a fully faithful functor

$$
H o\left(A-M_{o d}\right) \rightarrow H o(N(A)-M o d),
$$

whose essential image consists of all $N(A)$-dg-modules $E$ such that $H^{i}(E)=0$ for $i>0$ (see [25]).

Any $A \in$ sk-CAlg possesses a natural augmentation $A \rightarrow \pi_{0}(A)$, and thus we can define a base change morphism

$$
H o(N(A)-M o d) \rightarrow H o\left(\pi_{0}(A)-M o d\right) \simeq D\left(\pi_{0}(A)\right)
$$

Note that, by our conventions, $\pi_{0}(A)$ is considered here as a dg-algebra, and thus that $\pi_{0}(A)$-Mod denotes the category of $\pi_{0}(A)$-dg-modules, which is nothing else that the category of unbounded complexes of $\pi_{0}(A)$-modules. The category $D\left(\pi_{0}(A)\right)$ is thus the unbounded derived category of $\pi_{0}(A)$.

Definition 2.21. - An $N(A)$-dg-module $P$ is of Tor amplitude contained in $[a, b]$, if for any $\pi_{0}(A)$-module (non-dg) $M \in \operatorname{Mod}\left(\pi_{0}(A)\right)$, we have

$$
H^{i}\left(P \otimes_{N(A)}^{\mathbb{L}} M\right)=0 \quad \forall i \notin[a, b] .
$$

The following proposition characterizes the perfect dg-modules of Tor amplitude contained in $[a, b]$.

Proposition 2.22. - Let $A \in$ sk-CAlg, and $P$ and $Q$ be two perfect $N(A)$-dg-modules.

1. If $P$ (resp. $Q$ ) has Tor amplitude contained in $[a, b]$ (resp. $\left[a^{\prime}, b^{\prime}\right]$ ), then $P \otimes_{N(A)}^{\mathbb{L}} Q$ is a perfect $N(A)$-dg-module of Tor amplitude contained in $\left[a+a^{\prime}, b+b^{\prime}\right]$.

2. If $P$ and $Q$ have Tor amplitude contained in $[a, b]$, then for any morphism $f: P \rightarrow Q$, the homotopy fiber of $f$ has Tor amplitude contained in $[a, b+1]$.

3. The $N(A)$-dg-module $P$ has Tor amplitude contained in $[a, b]$ if and only if $P \otimes_{N(A)}^{\mathbb{L}}$ $\pi_{0}(A)$ is a perfect complex of $\pi_{0}(A)$-modules whose Tor amplitude is contained in $[a, b]$.

4. If $A \rightarrow A^{\prime}$ is a morphism in sk-CAlg, and if $P$ has Tor amplitude contained in $[a, b]$, then $P \otimes_{N(A)}^{\mathbb{L}} N\left(A^{\prime}\right)$ is a perfect $N\left(A^{\prime}\right)$-dg-module of Tor amplitude contained in $[a, b]$.

5. There exists $a \leqslant b$ such that $P$ has Tor amplitude contained in $[a, b]$.

6. If $P$ has Tor amplitude contained in $[a, a]$, then it is isomorphic in $\operatorname{Ho}(N(A)-M o d)$ to $E[-a]$, for some projective $N(A)$-module of finite type (see $[35, \S 1.2 .4])$.

7. If $P$ has Tor amplitude contained in $[a, b]$, then there exists a morphism

$$
E[-b] \rightarrow P,
$$

with $E$ a projective $N(A)$-dg-module of finite type whose homotopy cofiber has Tor amplitude contained in $[a, b-1]$.

Proof. - (1)-(5) are all easily deduced from the definition and the corresponding statement for perfect complexes of $\pi_{0}(A)$-modules, for which this is well known.

For (6), we can shift and thus reduce to the case where $a=0$. Let $P$ be a perfect $N(A)$-dg-module such that the complex of $\pi_{0}(A)$-modules $P \otimes_{N(A)}^{\mathbb{L}} \pi_{0}(A)$ is quasi-isomorphic to a $\pi_{0}(A)$-module projective and of finite type $E$ (considered as a complex concentrated in 
degree 0 ). As $E$ is projective, the quasi-isomorphism

$$
E[0] \rightarrow P \otimes_{N(A)}^{\mathbb{L}} \pi_{0}(A) \simeq \pi_{0}(P)
$$

can be lifted to a morphism of $N(A)$-dg-modules

$$
u: E^{\prime} \rightarrow P
$$

with $E^{\prime}$ a projective $N(A)$-dg-module of finite type. Let $C$ be the homotopy cofiber of the morphism $u$, which is a perfect $N(A)$-dg-module such that

$$
H^{i}\left(C \otimes_{N(A)}^{\mathbb{L}} \pi_{0}(A)\right) \simeq 0 \quad \forall i .
$$

This easily implies by an induction on $i$ that $H^{i}(C) \simeq 0$ for all $i$. Therefore, $C \simeq 0$ and thus $u$ is an equivalence, showing that $P$ is projective and of finite type.

Finally, for (7), let $E_{0}$ be a projective $\pi_{0}(A)$-module of finite type and

$$
u: E_{0}[-b] \rightarrow P \otimes_{N(A)}^{\mathbb{L}} \pi_{0}(A)
$$

be a morphism in $H o\left(\pi_{0}(A)-M o d\right)$, whose homotopy cofiber is of amplitude contained in $[a, b-1]$. As $E_{0}$ is a projective $\pi_{0}(A)$-module, it is possible to lift $u$ to a morphism

$$
v: E[-b] \rightarrow P
$$

with $E$ a projective $N(A)$-dg-module of finite type. The homotopy cofiber $C$ of $v$ is a perfect $N(A)$-dg-module, whose base change $C \otimes_{N(A)}^{\mathbb{L}} \pi_{0}(A)$ has Tor amplitude contained in $[a, b-1]$. By (3) this implies that $C$ has Tor amplitude contained in $[a, b-1]$.

\subsection{Zariski open and perfect modules}

We fix a simplicial commutative $k$-algebra $A \in s k$-CAlg, and a perfect $N(A)$-dg-module $P$. We define a sub- $D^{-}$-stack $V_{P}$ of $\mathbb{R} S p e c A$ as follows. For $A \rightarrow B$ a morphism in $s k-C A l g$, $V_{P}(B)$ is the full sub-simplicial set of $\overline{M a} p(A, B)$, consisting of morphisms $u: A \rightarrow B$ such that $P \otimes_{N(A)}^{\mathbb{L}} N(B)$ is quasi-isomorphic to 0 . The $D^{-}$-stack $V_{P}$ is called the complementary of the support of $P$. The purpose of this paragraph is to prove the following proposition, that will be needed in the sequel.

PROPOSITION 2.23. - The natural inclusion

$$
V_{P} \rightarrow \underline{R} \underline{\operatorname{Spec}} A
$$

is a quasi-compact Zariski open immersion.

Proof. - We consider $K=P \otimes_{N(A)}^{\mathbb{L}} \pi_{0}(A)$, which is a perfect complex of $\pi_{0}(A)$-modules. By the semi-continuity of the dimension of the cohomology groups of $K$, we know that there exists a quasi-compact Zariski open sub-scheme $U_{K} \subset S p e c \pi_{0}(A)$ with the property that for any commutative $k$-algebra $k^{\prime}$ and any morphism $u: \operatorname{Spec} k^{\prime} \rightarrow \operatorname{Spec}_{0}(A)$, the complex $K \otimes_{\pi_{0}(A)}^{\mathbb{L}} k^{\prime}$ is quasi-isomorphic to zero if and only if $u$ factors through $U_{K}$.

Let $\left(f_{1}, \ldots, f_{n}\right)$ be elements in $\pi_{0}(A)$ such that $U_{K}$ is the union of the standard Zariski open subschemes

$$
U_{i}:=\operatorname{Spec} \pi_{0}(A)\left[f_{i}^{-1}\right] \subset \operatorname{Spec} \pi_{0}(A)
$$

$4^{\mathrm{e}}$ SÉRIE - TOME $40-2007-\mathrm{N}^{\circ} 3$ 
We lift the elements $f_{i}$ to elements $g_{i} \in A_{0}$, and we consider the localized simplicial rings $A\left[g_{i}^{-1}\right]$, e.g. as defined in $[35, \S 1.2 .9]$. We let $V_{i}:=\mathbb{R} \underline{\operatorname{Spec}} A\left[g_{i}^{-1}\right]$, which is a Zariski open sub- $D^{-}$-stack of $\mathbb{R} S p e c A$. We define $V$ to be the quasi-compact Zariski open sub- $D^{-}$-stack of $\mathbb{R}$ Spec $A$ which is the union of the $V_{i}$.

We claim that the two sub-objects $V_{P}$ and $V$ of $\mathbb{R} S p e c A$ coincide, which will imply our proposition. Indeed, a morphism $u: A \rightarrow B$ in $s k-C A \overline{l g}$ belongs to $V(B)$ if and only if there exists an index $i$ such that the induced morphism $\pi_{0}(A) \rightarrow \pi_{0}(B)$ sends $f_{i}$ to an invertible element (see [35, §1.2.9]), at least locally for the étale topology on $B$. By the choice of the $f_{i}$, this is also equivalent to the fact that the complex

$$
K \otimes_{\pi_{0}(A)}^{\mathbb{L}} \pi_{0}(B) \simeq P \otimes_{N(A)}^{\mathbb{L}} \pi_{0}(B) \simeq\left(P \otimes_{N(A)}^{\mathbb{L}} N(B)\right) \otimes_{N(B)}^{\mathbb{L}} \pi_{0}(B)
$$

is quasi-isomorphic to zero. But, as $P$ is a perfect $N(A)$-dg-module, it is easy to check that $\left(P \otimes_{N(A)}^{\mathbb{L}} N(B)\right) \otimes_{N(B)}^{\mathbb{L}} \pi_{0}(B) \simeq 0$ if and only if $P \otimes_{N(A)}^{\mathbb{L}} N(B) \simeq 0$. Indeed, $P \otimes_{N(A)}^{\mathbb{L}} N(B)$ being perfect is bounded as a complex of $k$-modules. Therefore, if $P \otimes_{N(A)}^{\mathbb{L}} N(B)$ were not quasi-isomorphic to zero, we would choose $j$ to be the maximal index for which $H^{j}\left(P \otimes \mathbb{L}_{N(A)}^{\mathbb{L}}\right.$ $N(B)) \neq 0$, and we would have

$$
0 \neq H^{j}\left(\left(P \otimes_{N(A)}^{\mathbb{L}} N(B)\right) \otimes_{N(B)}^{\mathbb{L}} \pi_{0}(B)\right) \simeq H^{j}\left(P \otimes_{N(A)}^{\mathbb{L}} N(B)\right)=0 .
$$

\section{Moduli $D^{-}$-stacks associated to dg-categories}

This third section is the main body of the paper. We will start by the construction of a $D^{-}$-stack $\mathcal{M}_{T}$ associated to any dg-category $T$, and classifying pseudo-perfect $T^{\mathrm{op}}$-dg-modules. We will also provide a universal property of the $D^{-}$-stack $\mathcal{M}_{T}$ by showing that the construction $T \mapsto \mathcal{M}_{T}$ has an adjoint sending a $D^{-}$-stack $F$ to its dg-category of perfect complexes $L_{\mathrm{pe}}(F)$. The second paragraph is devoted to the proof of the fact that $\mathcal{M}_{T}$ is locally geometric and locally of finite type when $T$ is a smooth and proper dg-category. In a third paragraph we will study the sub- $D^{-}$-stack strongly of finite type of $\mathcal{M}_{T}$, and provide for each choice of a compact generator of $T$ an exhaustive family of such sub-stacks. Finally, in the last paragraph we present two examples of applications, perfect complexes on a smooth and proper scheme, and complexes of representations of a Quiver.

\subsection{Construction}

For any $A \in s k$-CAlg, we denote by $N(A)$ the $k$-dg-algebra obtained by normalization. We let $N(A)$-Mod be the $C(k)$-model category of (un-bounded) dg-modules over $N(A)$. For a morphism $A \rightarrow B$ in $s k$ - $C A l g$, there are an induced morphism $N(A) \rightarrow N(B)$ of dg-algebras, and an induced base change functor

$$
N(B) \otimes_{N(A)}-: N(A)-M o d \rightarrow N(B)-M o d
$$

which is a $C(k)$-enriched left Quillen functor. For simplicity we will also denote this functor by

$$
B \otimes_{A}-: N(A)-M o d \rightarrow N(B)-M o d .
$$

The construction $A \mapsto N(A)$-Mod is not functorial in $A$, and is only a lax functor. However, applying the standard strictification procedure, we can suppose that, up to an equivalence of 
categories, $A \mapsto N(A)$-Mod is a genuine functor from $s k$-CAlg to $C(k)$-model categories and $C(k)$-enriched left Quillen functor. We will still denote by $A \mapsto N(A)$-Mod this strictified object. We apply the construction $M \mapsto \operatorname{Int}(M)$ levelwise, and thus obtain a presheaf of $(\mathbb{V}$-small) dg-categories (note that all objects in $N(A)$-Mod are fibrant, so the base change functors preserve cofibrant and fibrant objects)

$$
\begin{aligned}
s k-C A l g & \rightarrow d g-C a t_{\mathbb{V}} \\
A & \mapsto \operatorname{Int}(N(A)-M o d) \\
(A \rightarrow B) & \mapsto B \otimes_{A}-.
\end{aligned}
$$

For any $A$, we let $\widehat{A}_{\text {pe }}$ be the full sub-dg-category of $\operatorname{Int}(N(A)-M o d)$ consisting of all perfect objects in the sense of $\$ 2.1$ (also called homotopically finitely presented). We first note that perfect objects are stable by the base change functors $B \otimes_{A}-$. Indeed, this follows formally from the definition of being homotopically finitely presented (see 2.1), from the existence of the Quillen adjunction

$$
B \otimes_{A}-: N(A)-\operatorname{Mod} \rightarrow N(B)-\operatorname{Mod} N(A)-M o d \leftarrow N(B)-\operatorname{Mod}: F,
$$

where $F$ is the forgetful functor which commutes with all homotopy colimits. We thus obtain a new presheaf of dg-categories

$$
\begin{aligned}
s k-C A l g & \rightarrow d g-C a t_{\mathbb{V}} \\
A & \mapsto \widehat{A}_{\mathrm{pe}} \\
(A \rightarrow B) & \mapsto B \otimes_{A}-.
\end{aligned}
$$

We now let $T$ be any dg-category and $T^{\mathrm{op}}$ be its opposite dg-category. We define a simplicial presheaf

$$
\mathcal{M}_{T}: s k-C A l g \rightarrow \text { SSet }
$$

by the formula

$$
\mathcal{M}_{T}(A):=\operatorname{Map}_{d g-C a t}\left(T^{\mathrm{op}}, \widehat{A}_{\mathrm{pe}}\right) .
$$

Here $M a p_{d g-C a t}$ denotes the mapping spaces of the model categories $d g$-Cat, which as any dg-category is fibrant will be taken to be

$$
\operatorname{Map}_{d g-C a t}\left(T, T^{\prime}\right):=\operatorname{Hom}\left(\Gamma^{*}(T), T^{\prime}\right)
$$

where $\Gamma^{*}$ is a co-simplicial resolution functor in the sense of $[9, \S 5]$. For $A \rightarrow B$ a morphism in $s k$-CAlg, the transition morphism

$$
\mathcal{M}_{T}(A) \rightarrow \mathcal{M}_{T}(B)
$$

is of course defined as the composition with $B \otimes_{A}-: \widehat{A}_{\mathrm{pe}} \rightarrow \widehat{B}_{\mathrm{pe}}$. This defines the simplicial presheaf $\mathcal{M}_{T}$.

Lemma 3.1. - The simplicial presheaf $\mathcal{M}_{T}$ is a $D^{-}$-stack in the sense of [35, Def. 1.3.2.1].

Proof. - Using the properties of the mapping spaces in model categories we see that it is enough to check the following three conditions (see [35] for more details).

$4^{\text {e }}$ SÉRIE - TOME $40-2007-\mathrm{N}^{\circ} 3$ 
- For any equivalence $A \rightarrow B$ in $s k-C A l g$, the induced morphism $\widehat{A}_{\mathrm{pe}} \rightarrow \widehat{B}_{\mathrm{pe}}$ is a quasiequivalence of dg-categories.

- For any two objects $A$ and $B$ in $s k-C A l g$, the natural morphism

$$
\widehat{A \times B}_{\mathrm{pe}} \rightarrow \widehat{A}_{\mathrm{pe}} \times \widehat{B}_{\mathrm{pe}}
$$

is a quasi-equivalence of dg-categories.

- For any étale hyper covering $X_{*} \rightarrow Y$ in $D^{-} A f f$, corresponding to a co-augmented cosimplicial object $A \rightarrow B_{*}$ in $s k-C A l g$, the induced morphism

$$
\widehat{A}_{\mathrm{pe}} \rightarrow \operatorname{Holim}_{[n] \in \Delta}\left(\widehat{B}_{n \mathrm{pe}}\right)
$$

is a quasi-equivalence of dg-categories.

The first of this property is satisfied because for an equivalence $A \rightarrow B$, the base change functor $B \otimes_{A}-$ is a Quillen equivalence, so induces a quasi-equivalence $\operatorname{Int}(N(A)$-Mod $) \rightarrow$ $\operatorname{Int}(N(B)-M o d)$, and thus a quasi-equivalence on the full sub-dg-categories of perfect objects. For the second and third properties, we first notice that it is enough to show that the induced morphisms

$$
\begin{gathered}
\operatorname{Int}(N(A) \times N(B)-M o d) \rightarrow \operatorname{Int}(N(A)-M o d) \times \operatorname{Int}(N(B)-M o d) \\
\operatorname{Int}(N(A)-M o d) \rightarrow \operatorname{Holim}_{[n] \in \Delta}\left(\operatorname{Int}\left(N\left(B_{n}\right)-M o d\right)\right)
\end{gathered}
$$

are quasi-equivalences, as being perfect is a local condition for the étale topology (see [35, Cor. 1.3.7.4]). The first of these two quasi-equivalences is clear.

The fact that the second one is a quasi-equivalence is a consequence of the main result of [30] and of the strictification theorem of [35, Appendix B]. Indeed, we need to prove that for any dg-category $C$, the morphism of simplicial sets

$$
\operatorname{Map}(C, \operatorname{Int}(N(A)-M o d)) \rightarrow \operatorname{Holim}_{[n] \in \Delta}\left(\operatorname{Map}\left(C, \operatorname{Int}\left(N\left(B_{n}\right)-M o d\right)\right)\right),
$$

is an equivalence. Applying the main theorem of [30], the simplicial set $\operatorname{Map}(C, \operatorname{Int}(N(A)$ $M o d)$ ) is equivalent to $N\left(\left(C \otimes^{\mathbb{L}} N(A)\right)-M_{o} d^{\text {cof }}\right)$, the nerve of the category of equivalences between cofibrant $C \otimes^{\mathbb{L}} N(A)$-dg-modules. In the same way, $\operatorname{Map}\left(C, \operatorname{Int}\left(N\left(B_{n}\right)-\operatorname{Mod}\right)\right)$ is equivalent to $N\left(\left(C \otimes^{\mathbb{L}} N\left(B_{n}\right)\right)-M o d^{\text {cof }}\right)$, the nerve of the category of equivalences between cofibrant $C \otimes^{\mathbb{L}} N\left(B_{n}\right)$-dg-modules. Finally, the fact that the morphism

$$
N\left(\left(C \otimes^{\mathbb{L}} N(A)\right)-\operatorname{Mod}^{\mathrm{cof}}\right) \rightarrow \operatorname{Holim}_{[n] \in \Delta}\left(N\left(\left(C \otimes^{\mathbb{L}} N\left(B_{n}\right)\right)-\operatorname{Mod}^{\mathrm{cof}}\right)\right)
$$

is an equivalence follows easily from the strictification theorem of [35, Appendix B] and faithfully flat cohomological descent (see [35, §1.3.7] for the similar example of the stack of quasi-coherent modules).

Definition 3.2. - The $D^{-}$-stack $\mathcal{M}_{T}$ is called the moduli stack of pseudo-perfect $T^{\mathrm{op}}{ }_{\text {-mo- }}$ dules.

By definition, the simplicial set $\mathcal{M}_{T}(k)$ is $\operatorname{Map}\left(T^{\mathrm{op}}, \widehat{k}_{\mathrm{pe}}\right)$, which by [30, §7] is equivalent to the nerve of the category of equivalences between $T^{\mathrm{op}}$-modules $E$, such that for any $x \in T^{\mathrm{op}}, E(x)$ is a perfect complex of $k$-modules. In other words, $\mathcal{M}_{T}(k)$ is a classifying space for pseudo-perfect $T^{\mathrm{op}}$-modules in the sense of Definition 2.7. In particular, the set $\pi_{0}\left(\mathcal{M}_{T}(k)\right)$ is in natural bijection with the isomorphism classes of pseudo-perfect $T^{\mathrm{op}}$-modules 
in $H o\left(T^{\mathrm{op}}-M o d\right)$. Moreover, for $x \in H o\left(T^{\mathrm{op}}-M o d\right)$ pseudo-perfect, one has natural group isomorphisms

$$
\pi_{1}\left(\mathcal{M}_{T}, x\right) \simeq \operatorname{Aut}(x, x), \quad \pi_{i}\left(\mathcal{M}_{T}, x\right) \simeq \operatorname{Ext}^{1-i}(x, x),
$$

where Aut and Ext's are computed in the triangulated category $H o\left(T^{\mathrm{op}}-M o d\right)$. This explains our choice of terminology in Definition 3.2.

Assume furthermore that $T$ is saturated in the sense of Definition 2.4, then the Yoneda embedding $\underline{h}: T \rightarrow \widehat{T}$ is quasi-fully faithful and its image consists of all pseudo-perfect (or equivalently perfect) $T^{\mathrm{op}}$-modules. In this case, Corollary 2.9 implies that the simplicial set $\mathcal{M}_{T}(k)$ is equivalent to $\operatorname{Map}(\mathbf{1}, T)$, which is a model for the classifying space of objects in $T$. More generally, for any $A \in s k-C A l g$, the simplicial set $\mathcal{M}_{T}(A)$ is equivalent to $\operatorname{Map}\left(\mathbf{1}, T \widehat{\otimes_{k}^{\mathbb{L}} N}(A)_{\mathrm{pe}}\right)$, and is a model for the classifying space of perfect $T^{\mathrm{op}} \otimes_{k}^{\mathbb{L}} N(A)$-dgmodules. In particular, the set $\pi_{0}\left(\mathcal{M}_{T}(A)\right)$ is in natural bijection with the set of isomorphism classes of perfect objects in $H o\left(\left(T \otimes_{k}^{\mathbb{L}} N(A)\right)^{\mathrm{op}}-M o d\right)$. Furthermore, for $E$ a perfect $T^{\mathrm{op}} \otimes_{k}^{\mathbb{L}}$ $N(A)$-module, there are a natural group isomorphisms

$$
\pi_{1}\left(\mathcal{M}_{T}(A), E\right) \simeq \operatorname{Aut}(E, E), \quad \pi_{i}\left(\mathcal{M}_{T}(A), E\right) \simeq E x t^{1-i}(E, E),
$$

where Aut and Ext's are computed in the triangulated category $H o\left(\left(T^{\mathrm{op}} \otimes_{k}^{\mathbb{L}} N(A)\right)-M o d\right)$. Therefore, when $T$ is saturated, the $D^{-}$-stack $\mathcal{M}_{T}$ classifies objects in $T$.

Clearly, the construction $T \mapsto \mathcal{M}_{T}$ is contravariantly functorial in $T$, and gives rise to a functor

$$
\begin{aligned}
d g-C a t^{\mathrm{op}} & \rightarrow k-D^{-} A f f^{\sim, \mathrm{et}} \\
T & \mapsto \mathcal{M}_{T} \\
\left(u: T \rightarrow T^{\prime}\right) & \mapsto\left(u^{*}: \mathcal{M}_{T^{\prime}} \rightarrow \mathcal{M}_{T}\right),
\end{aligned}
$$

from the model category of small dg-categories to the model category of $D^{-}$-stacks. For $u: T \rightarrow T^{\prime}$, and $A \in s k-C A l g$, the morphism $\mathcal{M}_{T^{\prime}} \rightarrow \mathcal{M}_{T}$ evaluated at $A$ is simply given by composition with $u$

$$
u^{*}: \operatorname{Map}\left(\left(T^{\prime}\right)^{\mathrm{op}}, \widehat{A}_{\mathrm{pe}}\right) \rightarrow \operatorname{Map}\left(T^{\mathrm{op}}, \widehat{A}_{\mathrm{pe}}\right) .
$$

By the properties of the mapping spaces (see $[9, \S 5]$ ) the functor $\mathcal{M}_{-}$sends quasi-equivalences between dg-categories to equivalences of $D^{-}$-stacks, and thus passes through the homotopy categories

$$
\begin{aligned}
H o(d g-C a t)^{\mathrm{op}} & \rightarrow D^{-} S t(k) \\
T & \mapsto \mathcal{M}_{T} \\
\left(u: T \rightarrow T^{\prime}\right) & \mapsto\left(u^{*}: \mathcal{M}_{T^{\prime}} \rightarrow \mathcal{M}_{T}\right) .
\end{aligned}
$$

As this last functor is obtained from an equivalence preserving functor, it is naturally compatible with the $H o(S S e t)$-enrichment of $H o(d g-C a t)^{\mathrm{op}}$ and $D^{-} S t(k)$. Explicitly, for $T$ and $T^{\prime}$ two dg-categories, the morphism in $\mathrm{Ho}(\mathrm{SSet})$

$$
\operatorname{Map}\left(T, T^{\prime}\right) \rightarrow \operatorname{Map}\left(\mathcal{M}_{T^{\prime}}, \mathcal{M}_{T}\right)
$$

is adjoint to

$$
\operatorname{Map}\left(T, T^{\prime}\right) \times \mathcal{M}_{T^{\prime}} \rightarrow \mathcal{M}_{T},
$$

$4^{\text {e }}$ SÉRIE - TOME $40-2007-\mathrm{N}^{\circ} 3$ 
which evaluated at $A \in s k$ - $C A l g$ is given by the composition of mapping spaces for the model category $d g$-Cat

$$
\operatorname{Map}\left(T, T^{\prime}\right) \times \operatorname{Map}\left(\left(T^{\prime}\right)^{\mathrm{op}}, \widehat{A}_{\mathrm{pe}}\right) \rightarrow \operatorname{Map}\left(T^{\mathrm{op}}, \widehat{A}_{\mathrm{pe}}\right) .
$$

The following lemma shows Morita invariance of the construction $T \mapsto \mathcal{M}_{T}$.

Lemma 3.3. - Let $T$ be any dg-category, and let us consider $\underline{h}: T \rightarrow \widehat{T}_{\mathrm{pe}}$ its Yoneda embedding. Then, the induced morphism in $D^{-} S t(k)$

$$
\underline{h}^{*}: \mathcal{M}_{\widehat{T}_{\mathrm{pe}}} \rightarrow \mathcal{M}_{T}
$$

is an isomorphism.

Proof. - This is a direct consequence of the definition of $\mathcal{M}_{T}$ and of the main results of $[30, \S 7]$.

By definition, if $T$ and $T^{\prime}$ are two $\mathbb{V}$-small dg-categories, the simplicial set $\operatorname{Map}\left(T, T^{\prime}\right)$ is itself $\mathbb{V}$-small. In particular, the object $\mathcal{M}_{T}$ exists in $D^{-} S t(k)$ even when $T$ is only a $\mathbb{V}$-small dg-categories. In other words, the functor $T \mapsto \mathcal{M}_{T}$ extends to a functor defined on all $\mathbb{V}$-small dg-categories

$$
\mathcal{M}_{-}: H o\left(d g-C a t_{\mathbb{V}}\right)^{\mathrm{op}} \rightarrow D^{-} S t(k) .
$$

Proposition 3.4. - The Ho(SSet)-enriched functor

$$
\mathcal{M}_{-}: H o\left(d g-C a t_{\mathbb{V}}\right)^{\mathrm{op}} \rightarrow D^{-} S t(k)
$$

has a Ho(SSet)-enriched left adjoint

$$
L_{\mathrm{pe}}: D^{-} S t(k) \rightarrow H o\left(d g-C a t_{\mathbb{V}}\right)^{\mathrm{op}} .
$$

Furthermore, for any $A \in s k-C A l g$, there exists a natural isomorphism in $H o\left(d g-C a t_{\mathbb{V}}\right)$

$$
L_{\mathrm{pe}}(\underline{\mathbb{R} S p e c} A) \simeq \widehat{A}_{\mathrm{pe}}
$$

Proof. - Let $F \in k-D^{-} A f f^{\sim, \text { et }}$, and let us write $F$ as a homotopy colimit of representable objects

$$
F \simeq \operatorname{Hocolim}_{i} h_{A_{i}},
$$

where $A_{i} \in$ sk-CAlg and $h_{A_{i}} \in k-D^{-} A f f^{\sim, \text { et }}$ is defined by $h_{A_{i}}(A):=\operatorname{Hom}\left(A_{i}, A\right)$. Note that this can be done functorially in $F \in k-D^{-} A f f^{\sim, \text { et }}$ by using for instance the standard free resolution of $F$. The diagram $i \mapsto h_{A_{i}}$ in $k-D^{-} A f f \sim^{\sim, \text { et }}$ gives a diagram $i \mapsto A_{i}$ in $s k$-CAlg by the Yoneda lemma. Applying the construction $A \mapsto \widehat{A}_{\text {pe }}$ (suitably strictified) we obtain a diagram $i \mapsto \widehat{A}_{i \mathrm{pe}}$ in $d g$-Cat. We set

$$
L_{\mathrm{pe}}(F):=\left(\operatorname{Holim}_{i}{\widehat{A_{i \mathrm{pe}}}}\right)^{\mathrm{op}} \in H o\left(d g-C a t_{\mathbb{V}}\right) .
$$

By definition, we have for any dg-category $T$ 


$$
\begin{aligned}
\operatorname{Map}_{d g-C^{\circ} \mathrm{op}}\left(L_{\mathrm{pe}}(F), T\right) & \simeq \operatorname{Holim}_{i} \operatorname{Map}_{d g-\operatorname{Cat}^{\mathrm{op}}}\left(\widehat{A}_{i \mathrm{pe}}, T^{\mathrm{op}}\right) \simeq \operatorname{Holim}_{i} \mathcal{M}_{T}\left(A_{i}\right) \\
& \simeq \operatorname{Holim}_{i} \operatorname{Map}\left(h_{A_{i}}, \mathcal{M}_{T}\right) \simeq \operatorname{Map}\left(\operatorname{Hocolim}_{i} h_{A_{i}}, \mathcal{M}_{T}\right) \\
& \simeq \operatorname{Map}\left(F, \mathcal{M}_{T}\right) .
\end{aligned}
$$

These are isomorphisms in $H o(S S e t)$, and are functorial in $T$. This implies that $F \mapsto L_{\mathrm{pe}}(F)$ as defined above is the left adjoint to $T \mapsto \mathcal{M}_{T}$.

Note that for a general object $F \in D^{-} S t(k)$, the dg-category $L_{\mathrm{pe}}(F)$ is not small in general, and only belongs to $\mathbb{V}$. However, when $F$ is equivalent to some small homotopy colimit of representable $D^{-}$-stacks, then $L_{\mathrm{pe}}(F)$ becomes equivalent to a small homotopy limit of small dg-categories, and thus is itself equivalent to a small dg-category. This is in particular the case when $F$ is $n$-geometric for some $n$.

Definition 3.5. - For a $D^{-}$-stack $F \in D^{-} S t(k)$, the dg-category $L_{\mathrm{pe}}(F) \in H o\left(d g-C a t_{\mathbb{V}}\right)$ is called the dg-category of perfect complexes on $F$.

\subsection{Geometricity}

The purpose of this paragraph is to provide a proof of the following theorem. Its proof will follow the main two steps we have used in the proof the algebraicity of the 1-stack $m_{C}$ in $\S 1$. We will first show that $\mathcal{M}_{1}$ is locally geometric and of finite presentation. Then, we will construct a morphism $\mathcal{M}_{T} \rightarrow \mathcal{M}_{1}$ and show that it is representable by identifying its fiber with dg-modules structures on a given perfect complex of $k$-modules.

THEOREM 3.6. - Let $T$ be a dg-category of finite type (see Definition 2.4). Then, the $D^{-}$stack $\mathcal{M}_{T}$ is locally geometric and locally of finite presentation.

Proof. - The proof of the theorem will take us some time, and will be divided in several propositions and lemmas.

The following proposition is a particular case where $T$ is the trivial dg-category 1 , which is obviously of finite type. In this case, we note that $\mathcal{M}_{1}$ is simply the $D^{-}$-stack of perfect modules. Indeed, for any $A \in s k-C A l g$, the simplicial set $\mathcal{M}_{\mathbf{1}}(A)$ is the nerve of the category of quasi-isomorphisms between cofibrant and perfect $N(A)$-dg-modules. In other words, $\mathcal{M}_{\mathbf{1}}(A)$ is a classifying space of perfect $N(A)$-dg-modules up to quasi-isomorphisms. The underived version of $\mathcal{M}_{1}$ has been considered in $[8, \S 21]$, and the following proposition is a derived analog of theorem [8, Thm. 21.5].

Proposition 3.7. - The $D^{-}$-stack $\mathcal{M}_{1}$ is locally geometric and locally of finite presentation.

Proof of Proposition 3.7. - The result of this proposition is somehow contained in [35], but we will reproduce the proof here for the reader's convenience.

Let $a, b \in \mathbb{Z}$ be two integers with $a \leqslant b$. We define a full sub- $D^{-}$-stack $\mathcal{M}_{1}^{[a, b]}$ of $\mathcal{M}_{\mathbf{1}}$ in the following way. For any $A \in s k-C A l g, \pi_{0}\left(\mathcal{M}_{1}(A)\right)$ can be identified with the quasi-isomorphism classes of perfect $N(A)$-dg-modules. We define $\mathcal{M}_{1}^{[a, b]}(A)$ to be the full sub-simplicial set of $\mathcal{M}_{1}(A)$ consisting of connected components corresponding to perfect $N(A)$-dg-modules of Tor amplitude contained in $[a, b]$ (see Definition 2.21). As the Tor amplitude is stable by base change (see Proposition 2.22), this defines a full sub- $D^{-}$-stack $\mathcal{M}_{1}^{[a, b]} \subset \mathcal{M}_{1}$. Furthermore, as any perfect $N(A)$-dg-module has a finite Tor amplitude (see Proposition 2.22), we find

$$
\mathcal{M}_{\mathbf{1}}=\bigcup_{a \leqslant b} \mathcal{M}_{1}^{[a, b]}
$$

$4^{\text {e }}$ SÉRIE - TOME $40-2007-\mathrm{N}^{\circ} 3$ 
In order to prove Proposition 3.7 it is then enough to show that each $\mathcal{M}_{1}^{[a, b]}$ is $n$-geometric and locally of finite presentation, for $n=b-a+1$.

We start by studying the diagonal.

LEMMA 3.8. - Let $X$ be a representable $D^{-}$-stack, and

$$
x: X \rightarrow \mathcal{M}_{1}^{[a, b]}, \quad y: X \rightarrow \mathcal{M}_{1}^{[a, b]}
$$

be two morphisms. Then, the $D^{-}$-stack $X \times_{\mathcal{M}_{1}^{[a, b]}}^{h} X$ is $(b-a)$-geometric and strongly of finite presentation.

Proof of Lemma 3.8. - We start by dealing directly with the case where $a=b$. In this case, $\mathcal{M}_{1}^{[a, a]}$ is equivalent to the $D^{-}$-stack of vector bundles (denoted by Vect $=\bigsqcup_{n}$ Vect $_{n}$ in [35]). Therefore, we know by [35] that $\mathcal{M}_{1}^{[a, a]}$ has a (-1)-representable diagonal of finite presentation. Therefore, $X \times{ }_{\mathcal{M}_{1}^{[a, b]}}^{h} X$ is a $(-1)$-representable $D^{-}$-stack of finite presentation and thus is 0 -geometric and strongly of finite presentation.

Let us now assume $a<b$. Let $A$ be an object in $s k$-CAlg representing $X$. The morphisms $x$ and $y$ correspond to $P$ and $Q$, two perfect $N(A)$-dg-modules of Tor amplitude contained in $[a, b]$. By [35, Appendix B], the $D^{-}$-stack $X \times_{\mathcal{M}_{1}^{[a, b]}}^{h} X \rightarrow X$ can be described by the functor

$$
\begin{aligned}
A / s k-C A l g & \rightarrow \text { SSet } \\
B & \mapsto M a p_{N(A)-M o d}^{\mathrm{eq}}\left(P, Q \otimes_{N(A)}^{\mathbb{L}} N(B)\right) .
\end{aligned}
$$

Let us consider the $D^{-}$-stack over $X$

$$
\begin{aligned}
F: A / s k-C A l g & \rightarrow S S e t \\
B & \mapsto \operatorname{Map}_{N(A)-M o d}\left(P, Q \otimes_{N(A)}^{\mathbb{L}} N(B)\right),
\end{aligned}
$$

together with the natural monomorphism

$$
j: X \times \times_{\mathcal{M}_{1}^{[a, b]}}^{h} X \hookrightarrow F .
$$

We first claim that $j$ is a 0-representable morphism. Indeed, for $B \in A / s k$-CAlg, and $u \in F(B)$, corresponding to a morphism $u: P \otimes_{N(A)}^{\mathbb{L}} N(B) \rightarrow Q \otimes_{N(A)}^{\mathbb{L}} N(B)$ of perfect $N(B)$-dgmodules, the $D^{-}$-stack

$$
j^{-1}(u):=\left(X \times_{\mathcal{M}_{1}^{[a, b]}}^{h} X\right) \times_{F}^{h} \underline{\mathbb{R} \underline{\operatorname{Spec}} B}
$$

is the full sub- $D^{-}$-stack of $\mathbb{R} S$ pec $B$ where $u$ becomes a quasi-isomorphism. This is the sub- $D^{-}$-stack of $\mathbb{R}$ Spec $B$ where the cone of $u$ is quasi-isomorphic to zero, which by Proposition 2.23 is a quasi-compact Zariski open sub- $D^{-}$-scheme of $\mathbb{R} S p e c B$. This implies that $j^{-1}(u)$ is a 0 -geometric $D^{-}$-stack.

In order to prove the lemma, it only remains to show that $F$ is an $(n-1)$-geometric $D^{-}$-stack. But $F$ can be written as

$$
\begin{aligned}
F: A / s k-C A l g & \rightarrow \text { SSet } \\
B & \mapsto \operatorname{Map}_{N(A)-M o d}(R, N(B)),
\end{aligned}
$$

where $R$ is the $N(A)$-dg-module $P \otimes_{N(A)}^{\mathbb{L}} Q^{\vee}$, of derived morphisms from $Q$ to $P$. Note that $R$ is a perfect $N(A)$-dg-module, and has Tor amplitude contained in $[a-b, b-a]$, as 
by assumptions $P$ and $Q$ are both of Tor amplitude contained in $[a, b]$ (see Proposition 2.22). Therefore Lemma 3.8 will be proved if we prove the following general sub-lemma.

Sub-LEMma 3.9. - Let $A \in$ sk-CAlg, and $R$ be a $N(A)$-dg-module which is perfect and of Tor amplitude contained in $[a, b]$, with $a, b \in \mathbb{Z}$. Then, the $D^{-}$-stack over $\mathbb{R} S p e c A$

$$
\begin{aligned}
F: A / s k-C A l g & \rightarrow S S e t \\
B & \mapsto \operatorname{Map}_{N(A)-M o d}(R, N(B)),
\end{aligned}
$$

is b-geometric and strongly of finite presentation.

Proof of Sub-lemma 3.9. - The proof is by induction on $b$. Let us assume that $b \leqslant 0$; then we have

$$
F \simeq \mathbb{R} \underline{\operatorname{Spec}} B
$$

where $B$ is the object of $A / s k$-CAlg defined as the derived free commutative $A$-algebra

$$
B:=\mathbb{R} \operatorname{Symm}_{A}(D(R)),
$$

where $D(R)$ is the simplicial $A$-module obtained by denormalizing $R$. As $D(R)$ is homotopically finitely presented as a simplicial $A$-module, $B$ is homotopically finitely presented as a commutative $A$-algebra. This implies that $F$ is representable and strongly of finite presentation.

Let us now assume that $b>0$. By Proposition 2.22, one can find a homotopy cofibration sequence of $N(A)$-dg-modules

$$
R \rightarrow R_{1} \rightarrow P[-b+1],
$$

where $P=N(A)^{r}$ is a free $N(A)$-dg-module, and $R_{1}$ is a perfect $N(A)$-dg-module of Tor amplitude contained in $[a, b-1]$. This gives rise to a fibration sequence of $D^{-}$-stacks

$$
K\left(\mathbb{G}_{a}, b-1\right)^{r} \rightarrow F_{1} \rightarrow F,
$$

where $F_{1}$ is defined as

$$
\begin{aligned}
F_{1}: A / s k-C A l g & \rightarrow S S e t \\
B & \mapsto \operatorname{Map}_{N(A)-M o d}\left(R_{1}, N(B)\right) .
\end{aligned}
$$

As the $D^{-}$-stack $K\left(\mathbb{G}_{a}, b-1\right)^{r}$ is $r$-geometric, strongly of finite presentation and smooth, the projection $F_{1} \rightarrow F$ is a covering, $(b-1)$-representable, strongly of finite presentation and smooth. By induction $F_{1}$ is known to be $(b-1)$-geometric and strongly of finite presentation. We deduce from this that $F$ can be obtained as the classifying $D^{-}$-stack of a $(b-1)$-smooth Segal groupoid $X_{*}$ such that each $X_{i}$ is strongly of finite presentation. This implies that $F$ is $b$-geometric and strongly of finite presentation (see [35]).

This finishes the proof of Lemma 3.8.

We come back to the proof of Proposition 3.7. It remains to show that the $D^{-}$-stack $\mathcal{M}_{1}^{[a, b]}$ has an $n$-atlas (for $n=b-a+1$ )

$$
U \rightarrow \mathcal{M}_{\mathbf{1}}^{[a, b]}
$$

with $U$ locally of finite presentation.

$4^{\text {e }}$ SÉRIE - TOME $40-2007-\mathrm{N}^{\circ} 3$ 
For this, we proceed by induction on the amplitude $n$. First of all, let us assume that $a=b$. Then, $\mathcal{M}_{1}^{[a, a]}$ is equivalent to the $D^{-}$-stack of vector bundles. The fact that this defines a 1 -geometric $D^{-}$-stack locally of finite presentation is well known (see [35]).

Let us now assume that $n>1$. By induction on $n$, we see that $\mathcal{M}_{1}^{[a, b-1]}$ is $(n-1)$-geometric locally of finite presentation. We define a $D^{-}$-stack $U$ in the following way. For $A \in s k-C A l g$, we consider $N(A)-\operatorname{Mod}^{(1)}$, the model category of morphisms in $N(A)$-Mod, endowed with its projective model structure (so that fibrations and equivalences are defined on the underlying objects in $N(A)$ - $M o d$, see [7, Thm. 11.6.1]). We restrict to cofibrant objects $u: Q \rightarrow R$ in $N(A)$ $\operatorname{Mod}^{(1)}$ such that $Q$ belongs to $\mathcal{M}_{1}^{[a, b-1]}(A)$, and $R$ belongs to $\mathcal{M}_{1}^{[b-1, b-1]}(A)$. This defines a full sub-category $\mathcal{C}(A)$ in $\left(N(A)-M_{o d} d^{(1)}\right)^{\text {cof }}$. Passing to the nerves of the sub-categories of equivalences gives a full sub-simplicial set $U(A):=N(w \mathcal{C}(A))$ of $N\left(w\left(N(A)-\operatorname{Mod}^{(1)}\right)^{\text {cof }}\right)$. When $A$ varies in $s k$ - $C A l g$, this defines (up to the standard strictification procedure) a simplicial presheaf

$$
\begin{aligned}
s k-C A l g & \rightarrow \text { SSet } \\
A & \mapsto U(A) .
\end{aligned}
$$

Lemma 3.10. - The $D^{-}$-stack $U$ defined above is $(n-1)$-geometric and locally of finite presentation.

Proof of Lemma 3.10. - We consider the morphism

$$
p: U \rightarrow \mathcal{M}_{1}^{[a, b-1]} \times^{h} \text { Vect }
$$

sending a morphism $u: Q \rightarrow R$ to the pair $(Q, R[b-1])$. We know by induction that $\mathcal{M}_{1}^{[a, b-1]} \times^{h}$ Vect is $(n-1)$-geometric and locally of finite presentation.

SuB-LEMma 3.11. - Let $M$ be the $C(k)$-enriched model category of $N(A)$-dg-modules, $M^{(1)}$ be the model category of morphisms in $M$, and $x$ and $y$ two objects in $M$. Then, there is a natural homotopy fiber square of simplicial sets

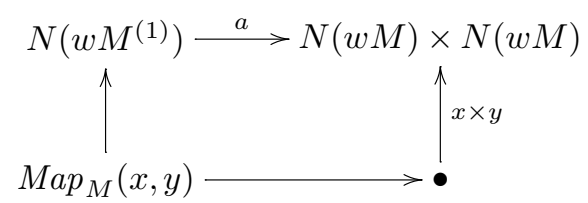

where $a$ is induced by the functor sending a morphism in $M$ to its domain and codomain.

Proof. - This follows from [22, Thm. 8.3].

The above sub-lemma implies that for $A \in s k-C A l g$, and $(Q, E) \in \mathcal{M}_{1}^{[a, b-1]}(A) \times \operatorname{Vect}(A)$, the $D^{-}$-stack

$$
p^{-1}(Q, E):=F \times_{\mathcal{M}_{1}^{h}}^{[a, b-1]} \times{ }^{h} \text { Vect } \underline{R} \underline{\operatorname{Spec}} A
$$

can be described as

$$
\begin{aligned}
p^{-1}(Q, E): A / s k-C A l g & \rightarrow S S e t \\
B & \mapsto M a p_{N(A)-M o d}\left(Q, E \otimes_{N(A)}^{\mathbb{L}} N(B)[-b+1]\right) .
\end{aligned}
$$


The $N(A)$-dg-module $R$ being of Tor amplitude contained in $[a, b-1], R \otimes_{N(A)}^{\mathbb{L}} E^{\vee}[b-1]$ is perfect and of Tor amplitude contained in $[a, 0]$. We deduce from Sub-lemma 3.9 that $p^{-1}(Q, R)$ is a representable $D^{-}$-stack locally of finite presentation. In other words, the morphism $p$ is representable and locally of finite presentation. This implies that the $D^{-}$-stack $U$ is itself $(n-1)$ representable and locally of finite presentation.

We now consider for any $A \in s k-C A l g$, the morphism

$$
U(A) \rightarrow \mathcal{M}_{1}(A)
$$

sending a morphism of $N(A)$-dg-module $u: Q \rightarrow R$ to its homotopy fiber $\operatorname{Hofib}(u)$. By the definition of $U(A)$, it is clear that $\operatorname{Hofib}(u)$ belongs to $\mathcal{M}_{1}^{[a, b]}(A) \subset \mathcal{M}_{\mathbf{1}}(A)$. When $A$ varies in $s k$ - $C A l g$, and using a model for the homotopy fiber which is compatible with base change, one gets a morphism of $D^{-}$-stacks

$$
\pi: U \rightarrow \mathcal{M}_{1}^{[a, b]} .
$$

LEMMA 3.12. - The morphism $\pi$ is an $(n-1)$-representable smooth covering.

Proof of Lemma 3.12. - We already know that $U$ is $(n-1)$-geometric locally of finite presentation, and that the diagonal of $\mathcal{M}_{1}^{[a, b]}$ is $(n-1)$-representable and locally of finite presentation. Therefore, the morphism $\pi$ is itself $(n-1)$-representable and locally of finite presentation.

Let us first prove that $\pi$ is a covering. Let $A \in s k-C A l g$, and $P \in \mathcal{M}_{1}^{[a, b]}(A)$. By definition, one can find a vector bundle $E$ on $\mathbb{R} S p e c A$, and a morphism $E[-b] \rightarrow P$, such that its homotopy cofiber $Q$ is of Tor amplitude contained in $[a, b-1]$. Therefore, there exists a homotopy fibration sequence

$$
P \rightarrow Q \rightarrow E[-b+1] .
$$

This implies by definition of $U$ and $\pi$ that $P$ is in the image of $\pi$. This shows that $\pi$ is a covering.

It remains to show that $\pi$ is smooth. For this, let $A \in s k-C A l g$, and $P$ be a point in $\mathcal{M}_{\mathbf{1}}^{[a, b]}$, corresponding to a morphism

$$
P: X:=\mathbb{R} \underline{\operatorname{Spec}} A \rightarrow \mathcal{M}_{1}^{[a, b]} .
$$

Let us denote by $\pi^{-1}(P)$ the $D^{-}$-stack over $X$ defined by

$$
\pi^{-1}(P):=U \times_{\mathcal{M}_{1}^{[a, b]}}^{h} X \rightarrow X .
$$

The diagonal of $\mathcal{M}_{1}^{[a, b]}$ being $(n-1)$-representable and locally of finite presentation, the $D^{-}$-stack $\pi^{-1}(P)$ is itself $(n-1)$-geometric and locally of finite presentation. We need to show that $\pi^{-1}(P)$ is smooth over $X$. We consider the morphism

$$
p: U \rightarrow \mathcal{M}_{1}^{[a, b-1]} \times{ }^{h} \text { Vect }
$$

as well as the induced morphism

$$
q: \pi^{-1}(P) \rightarrow U \times{ }^{h} X \rightarrow \mathcal{M}_{1}^{[a, b-1]} \times{ }^{h} \text { Vect } \times^{h} X \rightarrow \text { Vect } \times{ }^{h} X .
$$

$4^{\text {e }}$ SÉRIE - TOME $40-2007-\mathrm{N}^{\circ} 3$ 
As we know that the family of morphisms

$$
u_{r}: X \rightarrow \operatorname{Vect} \times^{h} X
$$

corresponding to the trivial rank $r$ vector bundle on $X$ (for various $r$ ), form a smooth atlas, we see that it is enough to check that the morphism

$$
V:=\pi^{-1}(P) \times_{\text {Vect } \times^{h} X}^{h} X \rightarrow X
$$

is smooth, for each of the morphism $u_{r}$. Let the rank $r$ be fixed; then by construction, the $D^{-}$-stack $V$ can be described in the following way

$$
\begin{aligned}
V: A / s k-C A l g & \rightarrow \text { SSet } \\
(A \rightarrow B) & \mapsto \operatorname{Map}_{N(B)-M o d}\left(B^{r}[-b+1], P \otimes_{N(A)}^{\mathbb{L}} N(B)[1]\right) .
\end{aligned}
$$

In other words, $V$ can be written as

$$
\begin{aligned}
V: A / s k-C A l g & \rightarrow \text { SSet } \\
(A \rightarrow B) & \mapsto \operatorname{Map}_{N(A)-M o d}(Q, N(B)),
\end{aligned}
$$

where $Q:=A^{r} \otimes_{N(A)}^{\mathbb{L}} P^{\vee}[-b]$. Now, let $u: Q \rightarrow N(B)$ be a morphism of $N(A)$-dg-modules, corresponding to commutative diagram of $D^{-}$-stacks

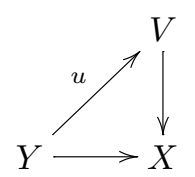

where $Y:=\mathbb{R}$ Spec $B$. For any simplicial $B$-module $M$, we have

$$
\operatorname{Der}_{u}(V / X, M) \simeq \operatorname{Map}_{N(A)-M o d}(Q, N(M)),
$$

where $N(M)$ is the normalized $N(A)$-dg-module associated to $M$. This shows that the relative cotangent complex of $V$ over $X$ at the point $u$, given by

$$
\mathbb{L}_{V / X, u} \simeq Q \otimes \otimes_{N(A)}^{\mathbb{L}} N(B) .
$$

As $Q=A^{r} \otimes_{N(A)}^{\mathbb{L}} P^{\vee}[-b]$ is of Tor amplitude contained in $[0, b-a]$ (see Proposition 2.22), the infinitesimal criterion for smoothness (see [35, §2.2.5]) tells us that $V \rightarrow X$ is smooth.

We have finished the proof of Lemma 3.12 and thus of Proposition 3.7.

We come back to the proof of Theorem 3.6. We start by recalling from Lemma 3.3 that the Yoneda embedding $\underline{h}: T \rightarrow \widehat{T}_{\mathrm{pe}}$ induces an isomorphism in $D^{-} S t(k)$

$$
\underline{h}^{*}: \mathcal{M}_{\widehat{T}_{\mathrm{pe}}} \simeq \mathcal{M}_{T}
$$

In particular, we can assume that $T$ is triangulated (see $2.6(1)$ ). We then choose a perfect generator $E$ of $\widehat{T}$, which as $T$ is triangulated is considered as an object in $T$. We consider the morphism of dg-categories

$$
i_{E}: \mathbf{1} \rightarrow T
$$


which classifies the object $E$. This morphism induces a morphism on the corresponding $D^{-}$-stacks

$$
\pi:=i_{E}^{*}: \mathcal{M}_{T} \rightarrow \mathcal{M}_{\mathbf{1}}
$$

By our Proposition 3.7, $\mathcal{M}_{1}$ is a locally geometric $D^{-}$-stack, locally of finite presentation. It can be written as a union

$$
\mathcal{M}_{\mathbf{1}}=\bigcup_{a \leqslant b} \mathcal{M}_{\mathbf{1}}^{[a, b]}
$$

where $\mathcal{M}_{1}^{[a, b]}$ denotes the full sub- $D^{-}$-stack of objects whose Tor amplitude is contained in $[a, b]$. We denote by $\mathcal{M}_{T}^{[a, b]}$ the full sub- $D^{-}$-stack of $\mathcal{M}_{T}$ defined by the following homotopy Cartesian square

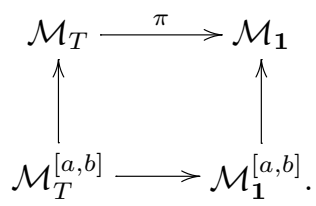

Clearly,

$$
\mathcal{M}_{T}=\bigcup_{a \leqslant b} \mathcal{M}_{T}^{[a, b]} .
$$

Therefore, in order to prove that $\mathcal{M}_{T}$ is locally geometric and locally of finite presentation, it is enough to prove that each $D^{-}$-stack $\mathcal{M}_{T}^{[a, b]}$ is $n$-geometric and locally of finite presentation, for some integer $n$ (depending on $a$ and $b$ ).

PROPOSITION 3.13. - Let $a \leqslant b$. There exists an integer $n$ such that the morphism

$$
\pi: \mathcal{M}_{T}^{[a, b]} \rightarrow \mathcal{M}_{1}^{[a, b]}
$$

is n-representable and strongly of finite presentation.

Proof of Proposition 3.13. - As $T$ is of finite type, we write $T$ as $\widehat{B_{\mathrm{pe}}^{\mathrm{op}}}$, for the dg-algebra $B=T(E, E)$ of endormophisms of the generator $E$. From our hypothesis on $T$ and Corollary $2.12, B$ is homotopically finitely presented in the model category $d g$-Alg.

We let $A \in$ sk-CAlg, $X:=\mathbb{R} \operatorname{Spec} A$, and $X \rightarrow \mathcal{M}_{1}^{[a, b]}$ be the morphism corresponding to a perfect $N(A)$-dg-module $P$ of Tor amplitude contained in $[a, b]$. We denote by $\mathcal{E}(P)$ the $N(A)$-dg-algebra of derived endomorphisms of $P$

$$
\mathcal{E}(P):=\underline{\mathbb{H o m}}_{N(A)}(P, P) .
$$

We let $\pi^{-1}(P)$ be the $D^{-}$-stack over $X$ defined by

$$
\pi^{-1}(P):=\mathcal{M}_{T}^{[a, b]} \times_{\mathcal{M}_{1}^{[a, b]}}^{h} X .
$$

We define a $D^{-}$-stack over $X$ in the following way

$$
\begin{aligned}
\underline{M a p}(B, \mathcal{E}(P)): A / s k-C A l g & \rightarrow \text { SSet } \\
\left(A \rightarrow A^{\prime}\right) & \mapsto M a p_{d g-A l g}\left(B, \mathcal{E}(P) \otimes_{N(A)}^{\mathbb{L}} N\left(A^{\prime}\right)\right) .
\end{aligned}
$$

$4^{\text {e }}$ SÉRIE - TOME $40-2007-\mathrm{N}^{\circ} 3$ 
Here $\mathcal{E}(P) \otimes_{N(A)}^{\mathbb{L}} N\left(A^{\prime}\right)$ is a $N\left(A^{\prime}\right)$-dg-algebra obtained by base change in the $N(A)$-dgalgebra $\mathcal{E}(P)$, and by considering it as a dg-algebra over $k$ by the forgetful functor.

LEMMA 3.14. - There exists an isomorphism in $D^{-} S t(X)$

$$
\pi^{-1}(P) \simeq \underline{\operatorname{Map}}(B, \mathcal{E}(P)) .
$$

Proof of Lemma 3.14. - As explained in $\$ 2.2$, we will consider dg-algebras as dg-categories with a unique object. Then, because of Lemma 3.3 $\mathcal{M}_{T} \simeq \mathcal{M}_{B}$ and the projection $\pi: \mathcal{M}_{B} \rightarrow$ $\mathcal{M}_{1}$ becomes induced by the unit morphism $k \rightarrow B$. The dg-algebra $\mathcal{E}(P) \otimes_{N(A)}^{\mathbb{L}} N\left(A^{\prime}\right)$, considered as a dg-category, can be identified with the full sub-dg-category of $\widehat{A_{\mathrm{pe}}^{\prime}}$ with $P \otimes_{N(A)^{\mathbb{L}}} N\left(A^{\prime}\right)$ a unique object. Therefore, for any $A^{\prime} \in A / s k$-CAlg, we find a diagram with homotopy Cartesian squares

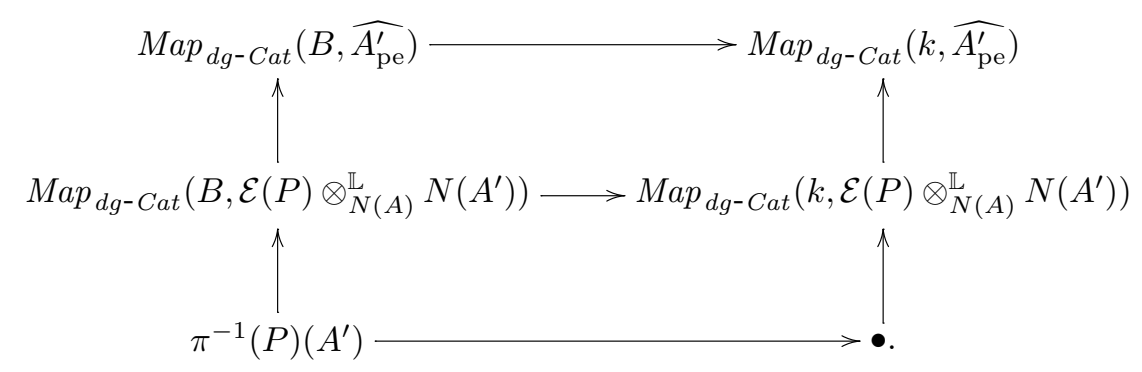

Let

$$
d g-A l g \underset{E}{\stackrel{G}{\rightleftarrows}} d g-C a t_{*}
$$

be the Quillen adjunction between the model category of dg-algebras and the model category of pointed dg-categories. It is easy to see that the left derived functor

$$
\mathbb{L} G: H o(d g-A l g) \rightarrow H o(d g-C a t)
$$

is fully faithful. Therefore, the functor $G$ also induces natural equivalences

$$
\operatorname{Map}_{d g-A l g}\left(B_{1}, B_{2}\right) \simeq \operatorname{Map}_{d g-C a t_{*}}\left(G\left(B_{1}\right), G\left(B_{2}\right)\right) .
$$

The diagram with homotopy Cartesian squares above tells us that $\pi^{-1}(P)\left(A^{\prime}\right)$ can be naturally identified with $M_{a p_{d g-C a t}}\left(B, \mathcal{E}(P) \otimes_{N(A)}^{\mathbb{L}} N\left(A^{\prime}\right)\right)$, and therefore with $\operatorname{Map}_{d g-A l g}(B, \mathcal{E}(P)$ $\left.\otimes_{N(A)}^{\mathbb{L}} N\left(A^{\prime}\right)\right)$. When $A^{\prime}$ varies in $A / s k$-CAlg, this provides an equivalence between $\pi^{-1}(P)$ and $\underline{\operatorname{Map}}(B, \mathcal{E}(P))$.

We come back to the proof of Proposition 3.13. Lemma 3.14 tells us that it is enough to show that the $D^{-}$-stack $\operatorname{Map}(B, \mathcal{E}(P))$ is $n$-geometric for some $n$, and that the projection $\operatorname{Map}(B, \mathcal{E}(P)) \rightarrow X$ is strongly of finite presentation.

Recall that the dg-algebra $B$ is homotopically finitely presented in the model category $d g-A l g$ of dg-algebras. Therefore, using Proposition $2.2 B$ is (equivalent to) a retract of some dg-algebra $B^{\prime}$, for which there exists a finite diagram

$$
B_{0}=k \longrightarrow B_{1} \longrightarrow \cdots \quad B_{i} \longrightarrow B_{i+1} \longrightarrow \cdots \longrightarrow B_{m}=B^{\prime},
$$


and such that for any $i$ there exists a homotopy push-out diagram

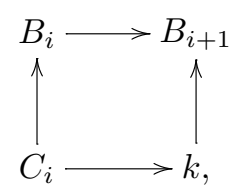

where $C_{i}$ is the free dg-algebra over the complex $k\left[p_{i}\right]$ for some integer $p_{i}$. Clearly, the morphism of $D^{-}$-stacks

$$
\underline{\operatorname{Map}}(B, \mathcal{E}(P)) \rightarrow X
$$

is retract of

$$
\underline{\operatorname{Map}}\left(B^{\prime}, \mathcal{E}(P)\right) \rightarrow X .
$$

As $n$-geometric $D^{-}$-stacks strongly of finite presentation are stable by retracts (see Lemma 2.15), it is enough to show that $\operatorname{Map}\left(B^{\prime}, \mathcal{E}(P)\right) \rightarrow X$ is $n$-representable for some $n$ and strongly of finite presentation. But, there exist a diagram of $D^{-}$-stacks over $X$

$$
\underline{\operatorname{Map}}\left(B^{\prime}, \mathcal{E}(P)\right) \rightarrow \cdots \rightarrow \underline{\operatorname{Map}}\left(B_{i+1}, \mathcal{E}(P)\right) \rightarrow \underline{\operatorname{Map}}\left(B_{i}, \mathcal{E}(P)\right) \rightarrow \cdots \underline{\operatorname{Map}}(k, \mathcal{E}(P))=X,
$$

as well as homotopy pull-back squares of $D^{-}$-stacks over $X$

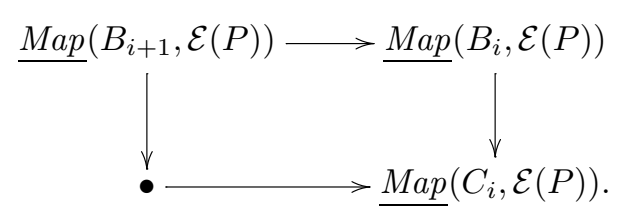

As $n$-geometric $D^{-}$-stacks strongly of finite presentations are stable by homotopy pull-backs (see Lemma 2.15), we see that it is enough to show that $\operatorname{Map}\left(C_{i}, \mathcal{E}(P)\right) \rightarrow X$ is $n$-geometric and strongly of finite presentation for some $n$. But, as $C_{i}$ is free over $k\left[p_{i}\right], \underline{M a p}\left(C_{i}, \mathcal{E}(P)\right)$ can be described, up to an equivalence, in the following terms

$$
\begin{aligned}
\operatorname{Map}\left(C_{i}, \mathcal{E}(P)\right): A / s k-C A l g & \rightarrow \text { SSet } \\
\left(A \rightarrow A^{\prime}\right) & \mapsto \operatorname{Map}_{C(k)}\left(k\left[p_{i}\right], \mathcal{E}(P) \otimes_{N(A)}^{\mathbb{L}} N\left(A^{\prime}\right)\right) .
\end{aligned}
$$

In other words, if we let $K$ be the $N(A)$-dg-module $\mathcal{E}(P)^{\vee}\left[p_{i}\right]$, one finds

$$
\begin{aligned}
\underline{\operatorname{Map}}\left(C_{i}, \mathcal{E}(P)\right): A / s k-C A l g & \rightarrow \text { SSet } \\
\left(A \rightarrow A^{\prime}\right) & \mapsto \operatorname{Map}_{C(k)}\left(K, N\left(A^{\prime}\right)\right) .
\end{aligned}
$$

Now, $K$ is of Tor amplitude contained in $\left[a-b-p_{i}, b-a-p_{i}\right]$, and thus Sub-lemma 3.9 tells us that

$$
\underline{\operatorname{Map}}\left(C_{i}, \mathcal{E}(P)\right) \rightarrow X
$$

is $\left(b-a-p_{i}\right)$-representable and strongly of finite presentation. This finishes the proof of Proposition 3.13, with the additional information that $n$ can be taken to be $\left(b-a-p_{i_{0}}\right)$, where $p_{i_{0}}$ is the lowest of the integers $p_{i}$.

Propositions 3.7 and 3.13, together with Lemma 2.20, achieve the proof of Theorem 3.6.

$4^{\text {e }}$ SÉRIE - TOME $40-2007-\mathrm{N}^{\circ} 3$ 
COROLlaRY 3.15. - Let $T$ be a smooth and proper dg-category; then $\mathcal{M}_{T}$ is a locally geometric $D^{-}$-stack locally of finite presentation.

Proof. - This follows from Corollary 2.13 and Theorem 3.6.

The following result is a corollary of the proof of Theorem 3.6 (more precisely of Proposition 3.13).

COROLLARY 3.16. - Let $T$ be a triangulated dg-category of finite type, and $E \in T$ a perfect generator classified by a morphism $i_{E}: \mathbf{1} \rightarrow T$, and let

$$
\pi:=i_{E}^{*}: \mathcal{M}_{T} \rightarrow \mathcal{M}_{\mathbf{1}}
$$

be the induced morphism. Then, for any $A \in$ sk-CAlg, any morphism

$$
x: X:=\mathbb{R} \underline{\operatorname{Spec}} A \rightarrow \mathcal{M}_{1},
$$

the induced morphism

$$
\mathcal{M}_{T} \times{ }_{\mathcal{M}_{\mathbf{1}}}^{h} X \rightarrow X
$$

is $n$-representable and strongly of finite presentation, for some $n$ depending on the choice of $X$ and the morphism $x$.

Proof. - This follows from Proposition 3.13, as any morphism $X \rightarrow \mathcal{M}_{1}$ factors through one of the $\mathcal{M}_{\mathbf{1}}^{[a, b]}$ for some $a \leqslant b$.

The above corollary almost says that $\pi$ is $n$-representable and strongly of finite presentation, except that the integer $n$ depends on the morphism $X \rightarrow \mathcal{M}_{1}$, and cannot be chosen uniformly.

Another important corollary of Theorem 3.6 is the following description of the tangent complexes of the $D^{-}$-stack $\mathcal{M}_{T}$. Recall from $[35,2.2]$ that $n$-geometric $D^{-}$-stacks have an obstruction theory, and in particular a (co)tangent complex. It is immediate to deduce that any locally geometric $D^{-}$-stack also has an obstruction theory and a global (co)tangent complex.

COROLLARY 3.17. - Let $T$ be a triangulated dg-category of finite type, and

$$
E: \text { Spec } k \rightarrow \mathcal{M}_{T}
$$

be a morphism corresponding to an object in $[T]$. Then, the tangent complex of $\mathcal{M}_{T}$ at the point $E$ is given by

$$
\mathbb{T}_{\mathcal{M}_{T}, E} \simeq T(E, E)[1]
$$

Proof. - Let

$$
E: *=\operatorname{Spec} k \rightarrow \mathcal{M}_{T}
$$

as in the statement. We consider

$$
\Omega_{E} \mathcal{M}_{T}:=* \times_{\mathcal{M}_{T}}^{h} *,
$$

the loop $D^{-}$-stack taken at E. Using [35, Appendix B], and [30], one has the following description

$$
\begin{aligned}
\Omega_{E} \mathcal{M}_{T}: s k-C A l g & \rightarrow S S e t \\
A & \mapsto \operatorname{Map}^{\prime}\left(k, T(E, E) \otimes_{k}^{\mathbb{L}} N(A)\right),
\end{aligned}
$$


where the $M a p^{\prime}$ is the full sub-simplicial set of $\operatorname{Map}_{C(k)}\left(k, T(E, E) \otimes_{k}^{\mathbb{L}} N(A)\right)$ corresponding to isomorphisms in

$$
\pi_{0}\left(M_{C(k)}\left(k, T(E, E) \otimes_{k}^{\mathbb{L}} N(A)\right)\right) \simeq\left[T \widehat{\otimes^{\mathbb{L}} N(A)}\right]\left(E \otimes^{\mathbb{L}} N(A), E \otimes^{\mathbb{L}} N(A)\right) .
$$

Therefore, $\Omega_{E} \mathcal{M}_{T}$ is a Zariski open sub- $D^{-}$-stack of

$$
\begin{aligned}
\underline{\operatorname{End}}(E): s k-C A l g & \rightarrow \operatorname{SSet} \\
A & \mapsto M a p\left(k, T(E, E) \otimes_{k}^{\mathbb{L}} N(A)\right) .
\end{aligned}
$$

Using the definition of derivations as in $[35, \S 1.2]$, it is easy to check that

$$
\mathbb{T}_{\underline{E n d}(E), i d} \simeq T(E, E)
$$

and so

$$
\mathbb{T}_{\Omega_{E} \mathcal{M}_{T}, *} \simeq T(E, E) .
$$

But, we have (see [35])

$$
\mathbb{T}_{\Omega_{E} \mathcal{M}_{T}, *} \simeq \mathbb{T}_{\mathcal{M}_{T}, E}[-1],
$$

and the required formula follows.

In order to finish this paragraph we mention the following easy, but interesting, consequence of Theorem 3.6. We let $T$ be a dg-category, and $I(1)$ be the free dg-category with two objects 0 and 1 and $I(1)(0,1)=k$ (i.e. $I(1)$ is the free dg-category of the category $\Delta(1)$, having two objects and a unique morphism between them). We set $T_{(1)}:=T \otimes I(1)$, and we consider the two morphisms

$$
s: T \rightarrow T_{(1)}, \quad t: T \rightarrow T_{(1)}
$$

induced by the two objects $0: 1 \rightarrow I(1)$ and $1: 1 \rightarrow I(1)$. Passing to the associated $D^{-}$-stacks, one gets two morphisms

$$
s: \mathcal{M}_{T_{(1)}} \rightarrow \mathcal{M}_{T}, \quad t: \mathcal{M}_{T_{(1)}} \rightarrow \mathcal{M}_{T} .
$$

Definition 3.18. - The $D^{-}$-stack $\mathcal{M}_{T_{(1)}}$ is called the $D^{-}$-stack of morphisms between pseudo-perfect dg-modules. It is denoted by $\mathcal{M}_{T}(1)$. The two morphisms

$$
s: \mathcal{M}_{T}(1) \rightarrow \mathcal{M}_{T}, \quad t: \mathcal{M}_{T}(1) \rightarrow \mathcal{M}_{T}
$$

are called source and target.

It is easy to check that if $T$ is of finite type then so is $T_{(1)}$. Therefore, from Theorem 3.6 we see that if $T$ is a dg-category of finite type then $\mathcal{M}_{T}(1)$ is a locally geometric $D^{-}$-stack. Furthermore, Sub-lemma 3.9 easily implies the following corollary.

COROLLARY 3.19. - Let T be a dg-category of finite type. Let us consider the morphism

$$
s \times t: \mathcal{M}_{T}(1) \rightarrow \mathcal{M}_{T} \times{ }^{h} \mathcal{M}_{T} .
$$

Then, for any $A \in$ sk-CAlg, any morphism

$$
x: X:=\mathbb{R} \underline{\text { Spec }} A \rightarrow \mathcal{M}_{T} \times{ }^{h} \mathcal{M}_{T},
$$

$4^{\mathrm{e}}$ SÉRIE - TOME $40-2007-\mathrm{N}^{\circ} 3$ 
the induced morphism

$$
\mathcal{M}_{T}(1) \times{ }_{\mathcal{M}_{T} \times h}^{h} \mathcal{M}_{T} X \rightarrow X
$$

is n-representable and strongly of finite presentation, for some $n$ depending on the choice of $X$ and the morphism $x$.

Proof. - Indeed, if the morphism $x$ corresponds to two $T^{\mathrm{op}} \otimes^{\mathbb{L}} N(A)$-dg-modules $P$ and $Q$, then the $D^{-}$-stack $\mathcal{M}_{T}(1) \times{ }_{\mathcal{M}_{T} \times{ }^{h} \mathcal{M}_{T}} X$ is given by

$$
\begin{aligned}
A / s k-C A l g & \rightarrow \text { SSet } \\
\left(A \rightarrow A^{\prime}\right) & \mapsto \operatorname{Map}_{N(A)-M o d}\left(K, N\left(A^{\prime}\right)\right),
\end{aligned}
$$

where $K:=P \otimes_{N(A)}^{\mathbb{L}} Q^{\vee}$ is the $N(A)$-dg-module of derived morphisms from $P$ to $Q$ (this follows from Sub-lemma 3.11). Sub-Lemma 3.9 implies the result.

\subsection{Sub-stacks strongly of finite presentation}

This section describes an exhaustive family of open sub- $D^{-}$-stacks $\mathcal{M}_{T}^{\nu} \subset \mathcal{M}_{T}$ which are strongly of finite presentation. This description is not canonical, as it depends on the choice of a perfect generator in $\widehat{T}$, but it will be useful in order to prove that certain $D^{-}$-stacks are strongly of finite presentation.

We let $\nu: \mathbb{Z} \rightarrow \mathbb{N}$ be a function with finite support (i.e. there is only a finite number of $i \in \mathbb{Z}$ with $\nu(i) \neq 0$ ). For $A \in s k$-CAlg, we define a full sub-simplicial set $\mathcal{M}_{\mathbf{1}}^{\nu}(A) \subset \mathcal{M}_{\mathbf{1}}(A)$ in the following way. A point $P \in \mathcal{M}_{\mathbf{1}}(A)$ belongs to $\mathcal{M}_{\mathbf{1}}^{\nu}(A)$ if for any field $K$ and any morphism $A \rightarrow \pi_{0}(A) \rightarrow K$ in $s k$-CAlg, we have

$$
\operatorname{Dim}_{K} H^{i}\left(P \otimes_{N(A)}^{\mathbb{L}} K\right) \leqslant \nu(i) \quad \forall i .
$$

This condition is stable by base change, and thus defines a full sub- $D^{-}$-stack

$$
\mathcal{M}_{1}^{\nu} \subset \mathcal{M}_{1}
$$

We start by noticing that if $a \leqslant b$ are such that $\nu(i)=0$ for all $i \notin[a, b]$, then

$$
\mathcal{M}_{1}^{\nu} \subset \mathcal{M}_{1}^{[a, b]} .
$$

Indeed, if $P \in \mathcal{M}_{\mathbf{1}}^{\nu}(A)$, then its base change $P^{\prime}:=P \otimes_{N(A)}^{\mathbb{L}} \pi_{0}(A)$ is a perfect complex of $\pi_{0}(A)$-modules whose fibers at all points in $\operatorname{Spec} \pi_{0}(A)$ are of Tor amplitude contained in $[a, b]$. This implies that $P^{\prime}$ has Tor amplitude contained in $[a, b]$, and thus that $P$ itself has Tor amplitude contained in $[a, b]$ (see Proposition 2.22).

Let $T$ be a triangulated dg-category of finite type, and let us choose a perfect generator $E \in T$. It is classified by a morphism $1 \rightarrow T$, which provides a morphism of $D^{-}$-stacks

$$
\pi: \mathcal{M}_{T} \rightarrow \mathcal{M}_{\mathbf{1}}
$$

We will denote by $\mathcal{M}_{T}^{\nu}$ the full sub- $D^{-}$-stack of $\mathcal{M}_{T}$ defined by the homotopy pull-back square

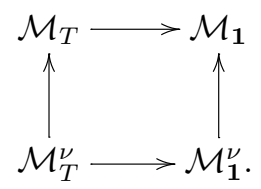


Note that $\mathcal{M}_{T}^{\nu}$ depends on the parameter $\nu$, but also on the choice of the generator $E$.

PROPOSITION 3.20. - Let $T$ be a triangulated dg-category of finite type, and $E$ and $\nu$ be as above. The $D^{-}$-stack $\mathcal{M}_{T}^{\nu}$ is n-geometric for some n, and strongly of finite presentation.

Proof. - There exists a diagram with homotopy Cartesian squares

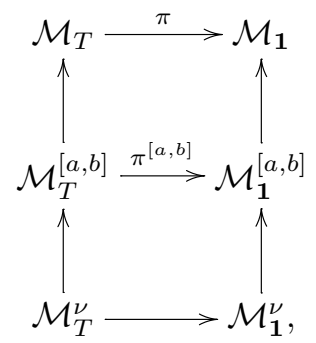

for some $a \leqslant b$ with $\nu(i)=0$ for all $i \notin[a, b]$. As we already know by 3.13 that $\pi^{[a, b]}$ is $n$-representable for some $n$ and strongly of finite presentation, then it is enough to show that the $D^{-}$-stack $\mathcal{M}_{1}^{\nu}$, which is known to be $(b-a+1)$-geometric, is strongly of finite presentation. By Lemma 3.8 we already know that the diagonal of $\mathcal{M}_{1}^{\nu}$ is strongly of finite presentation, and thus it only remains to show that $\mathcal{M}_{1}^{\nu}$ is quasi-compact. Equivalently, it is enough to find a quasi-compact and $n$-geometric $D^{-}$-stack $X$ and a covering $X \rightarrow \mathcal{M}_{1}^{\nu}$.

We proceed by induction on the length $l$ of $\nu$, where $l$ is the number of non-zero terms in the sequence $\nu$. For $l=1$, and say $\nu(i) \neq 0, \mathcal{M}_{1}^{\nu}$ is equivalent to the $D^{-}$-stack of vector bundles of rank less than $\nu(i)$. This is quasi-compact as it is covered by $\nu(i)$ copies of the final object $*$. Let us assume that all the $D^{-}$-stacks $\mathcal{M}_{1}^{\nu^{\prime}}$ are quasi-compact for all $\nu^{\prime}$ of length less than $l-1$, and let $\nu$ be of length $l$.

We define $\nu^{\prime}$ by

$$
\nu^{\prime}(i):=\nu(i) \quad \forall i<b-1, \quad \nu^{\prime}(b-1)=\nu(b-1)+\nu(b), \quad \nu^{\prime}(i)=0 \quad \forall i>b-1,
$$

where $b$ is the largest integer such that $\nu(b) \neq 0$. We define a $D^{-}$-stack $X$ in the following way. We first consider the $D^{-}$-stack of morphisms $\mathcal{M}_{1}(1)$ of Definition 3.18. We consider the full sub- $D^{-}$-stack $X$ of $\mathcal{M}_{\mathbf{1}}(1)$, whose objects over $A \in s k-C A l g$ consist of all objects $Q \rightarrow R$ in $\mathcal{M}_{\mathbf{1}}(1)(A)$, with $Q \in \mathcal{M}_{1}^{\nu^{\prime}}$ and $R$ of the form $E[-b+1]$, for $E$ a vector bundle of rank $\nu(b)$. We construct a morphism of $D^{-}$-stacks

$$
X \rightarrow \mathcal{M}_{1}^{[a, b]}
$$

by sending a morphism $Q \rightarrow R$ as above to its homotopy fiber. By 3.9, it is easy to see that the natural morphism sending $Q \rightarrow R$ to the pair $(Q, R[b-1])$

$$
X \rightarrow \mathcal{M}_{1}^{\nu^{\prime}} \times{ }^{h} \operatorname{Vect}_{\nu(b)}
$$

is $n$-representable, for some $n$, and strongly of finite presentation. By the induction assumption, this implies that $X$ is itself $n$-geometric for some $n$, and strongly of finite presentation.

$4^{\text {e }}$ SÉRIE - TOME $40-2007-\mathrm{N}^{\circ} 3$ 
Let us now consider the homotopy pull-back square

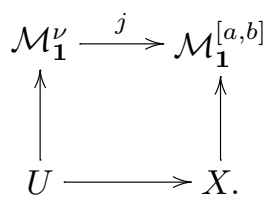

We first note that the morphism $j$ is a quasi-compact Zariski open immersion. Indeed, for $A \in s k-C A l g$, and $P$ a perfect $N(A)$-dg-module of Tor amplitude contained in $[a, b]$, the full sub- $D^{-}$-stack of $\mathbb{R}$ Spec $A$ where $P$ lies in $\mathcal{M}_{1}^{\nu}(A)$ is a Zariski open sub- $D^{-}$-scheme, corresponding to the open sub-scheme of $\operatorname{Spec} \pi_{0}(A)$ where $P \otimes_{N(A)}^{\mathbb{L}} \pi_{0}(A)$ lies in $\mathcal{M}_{\mathbf{1}}^{\nu}\left(\pi_{0}(A)\right)$. But, for a given perfect complex $Q$ of $k^{\prime}$-modules, for some commutative ring $k^{\prime}$, the sub-set of points $x \in S p e c k^{\prime}$ such that $\operatorname{Dim}_{k^{\prime}(x)} H^{i}\left(Q \otimes_{k}^{\mathbb{L}} k^{\prime}(x)\right) \leqslant \nu(i)$ for all $i$, is a quasi-compact open sub-set by semi-continuity. Therefore, the $D^{-}$-stack $U$ is also $n$-geometric and strongly quasicompact. It remains to show that the morphism

$$
U \rightarrow \mathcal{M}_{1}^{\nu}
$$

is a covering, or equivalently that any $P \in \mathcal{M}_{1}^{\nu}(A)$ is, locally on $A$, in the image of $X \rightarrow \mathcal{M}_{1}^{[a, b]}$.

Let $P$ be a point in $\mathcal{M}_{1}^{\nu}(A)$, and $A \rightarrow \pi_{0}(A) \rightarrow K$ a morphism with $K$ a field, corresponding to a point $x \in \operatorname{Spec} \pi_{0}(A)$. We can choose a morphism

$$
K^{\nu(b)}[-b] \rightarrow P \otimes_{N(A)}^{\mathbb{L}} K
$$

inducing a surjective morphism

$$
g: K^{\nu(b)} \rightarrow H^{b}\left(P \otimes_{N(A)}^{\mathbb{L}} K\right) .
$$

As $H^{i}(P)=0$ for all $i>b$, we have

$$
H^{b}(P) \otimes_{\pi_{0}(A)} K \simeq H^{b}\left(P \otimes_{N(A)}^{\mathbb{L}} K\right),
$$

and thus the morphism $g$ extends to a morphism of $N(A)$-dg-modules

$$
u: A^{\nu(b)}[-b] \rightarrow P
$$

We denote by $Q$ the homotopy cofiber of $u$, and we consider the induced morphism $Q \rightarrow A^{\nu(b)}[-b+1]$. The morphism $H^{b}(u)$ being surjective at the point $x$, the object $Q$ lies in $\mathcal{M}_{1}^{\nu^{\prime}}\left(A^{\prime}\right)$, for $A \rightarrow A^{\prime}$ a Zariski open neighborhood around $x$. This shows that $P \otimes_{N(A)}^{\mathbb{L}} N\left(A^{\prime}\right)$ lies in the image of $X\left(A^{\prime}\right)$. As the point $x$ was arbitrary, this finishes the proof that $U \rightarrow \mathcal{M}_{1}^{\nu}$ is a covering, and thus that $\mathcal{M}_{1}^{\nu}$ is quasi-compact.

\subsection{Some consequences}

Let $T$ be a dg-category of finite type and $\mathcal{M}_{T}$ be the $D^{-}$-stack of pseudo-perfect $T^{\mathrm{op}}$-dgmodules. We consider the truncation $t_{0} \mathcal{M}_{T}$, and we define for any integer $n>0$ a substack $t_{0} \mathcal{M}_{T}^{n \text {-rid }} \subset t_{0} \mathcal{M}_{T}$ of $n$-rigid objects in the following way: for any $k$-algebra $k^{\prime} \in k$-CAlg, the 
simplicial set $t_{0} \mathcal{M}_{T}^{n \text {-rig }}\left(k^{\prime}\right)$ is defined to be the full sub-simplicial set of $t_{0} \mathcal{M}_{T}\left(k^{\prime}\right)=\mathcal{M}_{T}\left(k^{\prime}\right)$ consisting of $T^{\mathrm{op}} \otimes^{\mathbb{L}} k^{\prime}$-dg-modules $E$ such that for any morphism $k^{\prime} \rightarrow k^{\prime \prime}$ in $k$-CAlg we have

$$
\begin{aligned}
& E x t_{T^{\mathrm{op}} \otimes^{\mathbb{L}} k^{\prime \prime}-M o d}\left(E \otimes_{k^{\prime}}^{\mathbb{L}} k^{\prime \prime}, E \otimes_{k^{\prime}}^{\mathbb{L}} k^{\prime \prime}\right)=\pi_{i}\left(\operatorname{Map}_{T^{\mathrm{op}} \otimes^{\mathbb{L}} k^{\prime \prime}-M o d}\left(E \otimes_{k^{\prime}}^{\mathbb{L}} k^{\prime \prime}, E \otimes_{k^{\prime}}^{\mathbb{L}} k^{\prime \prime}\right)\right)=0 \\
& \quad \forall i \geqslant n .
\end{aligned}
$$

COROLlaRY 3.21. - The stack $t_{0} \mathcal{M}_{T}^{n \text {-rid }}$ is an Artin n-stack. In particular $t_{0} \mathcal{M}_{T}^{1 \text {-rid }}$ is an Artin 1-stack.

Proof. - By definition and by the description of homotopy groups of nerves of categories of equivalences in model categories (see [35]), the stack $t_{0} \mathcal{M}_{T}^{n \text {-rid }}$ is an $n$-stack. Moreover, by semi-continuity of the dimension of the cohomology groups of perfect complexes, it is easy to see that the inclusion morphism $t_{0} \mathcal{M}_{T}^{n \text {-rid }} \rightarrow t_{0} \mathcal{M}_{T}$ is a Zariski open immersion. This implies that $t_{0} \mathcal{M}_{T}^{n \text {-rid }}$ is a locally geometric stack which is $n$-truncated, and thus that it is an Artin $n$-stack by Lemma 2.19 .

We define a full substack $t_{0} \mathcal{M}_{T}^{\text {simp }} \subset t_{0} \mathcal{M}_{T}^{1 \text {-rig }}$, of simple objects, as follows: for any $k$-algebra $k^{\prime} \in k$-CAlg, the simplicial set $t_{0} \mathcal{M}_{T}^{\text {simp }}\left(k^{\prime}\right)$ is defined to be the full sub-simplicial set of $t_{0} \mathcal{M}_{T}\left(k^{\prime}\right)^{1 \text {-rig }}$ consisting of $T^{\mathrm{op}} \otimes^{\mathbb{L}} k^{\prime}$-dg-modules $E$ such that for any morphism $k^{\prime} \rightarrow k^{\prime \prime}$ in $k$-CAlg the natural morphism

$$
k^{\prime \prime} \rightarrow E n d_{H o\left(T^{\mathrm{op}} \otimes^{\mathbb{L}} k^{\prime \prime}-M o d\right)}\left(E \otimes_{k^{\prime}}^{\mathbb{L}} k^{\prime \prime}\right)
$$

is an isomorphism.

COROLlary 3.22. - The stack $t_{0} \mathcal{M}_{T}^{\text {simp }}$ is an Artin 1-stack. Moreover, the sheaf $\pi_{0}\left(t_{0} \mathcal{M}_{T}^{\text {simp }}\right)$ is an algebraic space locally of finite presentation, and the natural morphism

$$
t_{0} \mathcal{M}_{T}^{\text {simp }} \rightarrow \pi_{0}\left(t_{0} \mathcal{M}_{T}^{\text {simp }}\right)
$$

is a locally trivial fibration (for the étale topology) with fibers $K\left(\mathbb{G}_{m}, 1\right)$.

Proof. - It is easy to see that $t_{0} \mathcal{M}_{T}^{\text {simp }}$ is an open substack in $t_{0} \mathcal{M}_{T}^{1 \text {-rig }}$ and so is an Artin 1-stack by Corollary 3.21. Moreover, let $M:=\pi_{0}\left(t_{0} \mathcal{M}_{T}^{\text {simp }}\right)$. It is easy to see that the natural morphism

$$
p: t_{0} \mathcal{M}_{T}^{\text {simp }} \rightarrow M
$$

is a torsor over the group stack $K\left(\mathbb{G}_{m}, 1\right)$. This implies that for any affine $k$-scheme $Y$ and any morphism $Y \rightarrow M$, the induced morphism

$$
t_{0} \mathcal{M}_{T}^{\text {simp }} \times_{M}^{h} Y \rightarrow Y
$$

is locally (for the étale topology) equivalent on $Y$ to the projection $Y \times K\left(\mathbb{G}_{m}, 1\right) \rightarrow Y$. This implies that the morphism $p$ is 1-representable, smooth and surjective. By [35] this implies that $M$ is $n$-geometric for some $n$, and thus is an Artin 0 -stack, or in other words an algebraic space. Moreover, as $M$ is locally a retract of $t_{0} \mathcal{M}_{T}^{\text {simp }}, M$ is also locally of finite presentation.

Remark 3.23. - For any $n$-geometric $D^{-}$-stack $F$, the natural closed embedding $t_{0}(F) \rightarrow F$ is a formal thickening and induces an equivalence between the small Zariski sites of $F$ and of $t_{0}(F)$. Therefore, the open substacks

$$
t_{0} \mathcal{M}_{T}^{\text {simp }} \subset t_{0} \mathcal{M}_{T}^{n \text {-rig }} \subset t_{0} \mathcal{M}_{T}
$$

$4^{\text {e }}$ SÉRIE - TOME $40-2007-\mathrm{N}^{\circ} 3$ 
correspond to open sub- $D^{-}$-stacks

$$
\mathcal{M}_{T}^{\text {simp }} \subset \mathcal{M}_{T}^{n \text {-rig }} \subset \mathcal{M}_{T}
$$

This provides natural derived version of the stacks $t_{0} \mathcal{M}_{T}^{\text {simp }}$ and $t_{0} \mathcal{M}_{T}^{n \text {-rig }}$.

Let $T$ be a saturated dg-category. We define a presheaf of groups on the site of affine $k$-schemes with the étale topology as follows:

$$
\begin{aligned}
& k-C A l g \rightarrow G p \\
& k^{\prime} \mapsto A u t_{H o\left(d g-C a t_{k^{\prime}}\right)}\left(\widehat{T^{\mathrm{op} \otimes^{\mathbb{L}}}} k^{\prime}\right) \simeq A u t_{H o\left(d g-C a t_{k^{\prime}}\right)}\left(T^{\mathrm{op} \otimes^{\mathbb{L}}} k_{\mathrm{pe}}^{\prime}\right),
\end{aligned}
$$

where $\mathrm{Ho}\left(d g\right.$-Cat $\left.t_{k^{\prime}}\right)$ is the homotopy category of $k^{\prime}$-dg-categories, and $k$-CAlg is the category of commutative $k$-algebras. We will denote by $\operatorname{aut}(T)$ the associated sheaf of groups.

In order to state the next corollary remind from [30] that for any dg-category $T$ it is possible to define a Hochschild complex $H H(T)$ whose cohomology groups are given by

$$
H H^{i}(T)=\operatorname{Ext}_{T \otimes^{\mathbb{L}} T^{\mathrm{op}}}^{i}(T, T) \simeq H^{i}(\mathbb{R} \underline{\operatorname{Hom}}(T, T)(\mathrm{id}, \mathrm{id})) .
$$

COROLLARY 3.24. - Assume that one of the two conditions below are satisfied

1. We have $H H^{i}(T)=0$ for all $i<0$ and the natural morphism $k \rightarrow H H^{0}(T)$ is an isomorphism.

2. The ring $k$ is a field.

Then the sheaf of groups aut $(T)$ is representable by an algebraic space locally of finite presentation over $k$ (and thus by a scheme if $k$ is a field).

Proof. - We start to define a stack

$$
\begin{aligned}
\underline{\operatorname{End}}(T): k-C A l g & \rightarrow \text { SSet } \\
k^{\prime} & \mapsto M a p_{d g-C a t_{k^{\prime}}}\left(\widehat{T \mathbb{Q L}_{\mathrm{pe}}^{\prime}}, \widehat{T \mathbb{Q ⿻}^{\mathbb{L}} k_{\mathrm{pe}}^{\prime}}\right) .
\end{aligned}
$$

Using that $T$ is saturated and [30], it is easy to see that $\operatorname{End}(T)$ is equivalent to $t_{0} \mathcal{M}_{T \otimes^{\mathbb{L}} T^{\mathrm{op}}}$. Therefore, it is a locally geometric stack by Theorem 3.6. We can also define a substack $\underline{A u t}(T)$ of $\underline{\operatorname{End}}(T)$ as follows:

$$
\begin{aligned}
\underline{A u t}(T): k-C A l g & \rightarrow S S e t \\
k^{\prime} & \mapsto M a p_{d g-C a t_{k^{\prime}}}^{\mathrm{eq}}\left(\widehat{T \mathbb{Q ⿻}^{\mathbb{L}} k_{\mathrm{pe}}^{\prime}}, \widehat{T \mathbb{Q ⿻}^{\mathbb{L}} k_{\mathrm{pe}}^{\prime}}\right) .
\end{aligned}
$$

LEMMA 3.25. - Let $B$ be a smooth and proper dg-algebra over $k$. Let $E$ be a perfect $B \otimes \mathbb{L} B^{\mathrm{op}}$-dg-module. Then, the induced morphism of dg-categories

$$
F_{E}: \widehat{B_{\mathrm{pe}}^{\mathrm{op}}} \rightarrow \widehat{B_{\mathrm{pe}}^{\mathrm{op}}}
$$

is a quasi-equivalence if and only if the two natural morphisms of complexes of $k$-modules

$$
\begin{gathered}
B \rightarrow \underline{\mathbb{R}}_{\text {End }}^{B-M o d}(E, E) \\
E \otimes \mathbb{L}_{B} \underline{\underline{E n d}}_{B-M o d}(E, B) \rightarrow B
\end{gathered}
$$

are quasi-isomorphisms. 
Proof of the lemma. - The functor $F_{E}$ sends a $B$-dg-module $X$ to $E \otimes_{B}^{\mathbb{L}} X$. This implies that $F_{E}$ is fully faithful if and only if the morphism

$$
B \rightarrow \mathbb{R} \underline{\operatorname{End}}_{B-M o d}(E, E)
$$

is a quasi-isomorphism (because $B$ is a generator of the category of $B$-dg-modules). The functor $F_{E}$ has a right adjoint $G_{E}$ sending a $B$-dg-module $X$ to $\mathbb{R} \underline{E n d}_{B-M o d}(E, X)$. Therefore, $G_{E}$ is fully faithful if and only if the morphism

$$
E \otimes \otimes_{B}^{\mathbb{L}} \mathbb{R} \underline{\operatorname{End}}_{B-M o d}(E, B) \rightarrow B
$$

is a quasi-isomorphism.

Lemma 3.25, and the semi-continuity of the dimension of the cohomology groups of perfect complexes, imply that the natural embedding

$$
\underline{\operatorname{Aut}}(T) \rightarrow \underline{\operatorname{End}}(T)
$$

is a Zariski open immersion (this follows from Proposition 2.23 applied to cones of the two morphisms of Lemma 3.25). In particular, $\operatorname{Aut}(T)$ is a locally geometric stack.

By definition $\operatorname{aut}(T)$ is the sheaf $\pi_{0}(\underline{\operatorname{Aut}}(T))$. Let $p: \underline{\operatorname{Aut}}(T) \rightarrow \operatorname{aut}(T)$ be the natural morphism, and let us prove that $p$ is $n$-representable for some $n$, and smooth. By [35, Cor. 1.3.4.5] this will imply that $\operatorname{aut}(T)$ is an algebraic space locally of finite presentation. For this, let $X=$ Spec $k^{\prime}$ be an affine $k$-scheme and $X \rightarrow$ aut $(T)$ be a morphism. We want to prove that the stack $\underline{\operatorname{Aut}}(T) \times{ }_{\text {aut }(T)}^{h} X$ is $n$-geometric for some $n$ and that the morphism $\underline{A u t}(T) \times{ }_{a u t(T)}^{h} X \rightarrow X$ is smooth. As this is local on $X$, we can assume that the morphism $X \rightarrow \operatorname{aut}(T)$ lifts to a morphism $X \rightarrow \underline{A u t}(T)$.

The morphism $X=\operatorname{Spec} k^{\prime} \rightarrow \underline{A u t}(T)$ corresponds to a certain perfect $T \otimes^{\mathbb{L}} T^{\mathrm{op}}$-dgmodule $E$. By definition, the stack $\underline{A u t}(T) \times_{\text {aut }(T)}^{h} X$ can be written as $K(\underline{A u t}(E), 1)$, where $\underline{\operatorname{Aut}}(E)$ is the group stack defined by

$$
\begin{aligned}
\underline{A u t}(E): k^{\prime}-C A l g & \mapsto S S e t \\
k^{\prime \prime} & \mapsto M a p_{T \otimes^{\mathbb{L}} T^{\mathrm{eq}} \otimes^{\mathbb{L}} k^{\prime \prime}}\left(E \otimes^{\mathbb{L}} k^{\prime \prime}, E \otimes^{\mathbb{L}} k^{\prime \prime}\right) .
\end{aligned}
$$

Therefore, it is enough to show that $\underline{A u t}(E)$ is an $n$-geometric stack for some $n$ and is smooth over $X$. The fact that $\underline{A u t}(E)$ is $n$-geometric for some $n$ follows from the local geometricity of $t_{0} \mathcal{M}_{T \otimes^{\mathbb{L}} T^{\text {op }}}$, as we have

$$
\underline{A u t}(E) \simeq X \times_{t_{0} \mathcal{M}_{T \otimes^{\mathbb{L}} T \text { op }}^{h}} X .
$$

It remains to show that it is smooth over $X$.

As $\underline{A u t}(T)$ is a group stack, the stack $\underline{A u t}(E)$ is equivalent to $\underline{A u t}(I)$, where $I$ is $T \otimes^{\mathbb{L}} k^{\prime}$ considered as a $T \otimes^{\mathbb{L}} T^{\mathrm{op}} \otimes^{\mathbb{L}} k^{\prime}$-dg-module. The stack $\underline{A u t}(I)$ is a Zariski open substack of $\underline{\operatorname{End}}(I)$, defined naturally by

$$
\begin{aligned}
\underline{\operatorname{End}}(I): k^{\prime}-C A l g & \mapsto S S e t \\
k^{\prime \prime} & \mapsto M a p_{T \otimes^{\mathbb{L}} T^{\mathrm{op}} \otimes^{\mathbb{L}} k^{\prime \prime}}\left(T \otimes^{\mathbb{L}} k^{\prime \prime}, T \otimes^{\mathbb{L}} k^{\prime \prime}\right) .
\end{aligned}
$$

It only remains to show that $\underline{E n d}(I)$ is smooth over $X$. But, under condition (1), we have $\underline{\operatorname{End}}(I) \simeq \mathbb{G}_{a}$. And under condition (2), it is easy to see that $\underline{\operatorname{End}}(I)$ is equivalent, as a stack

$4^{\text {e }}$ SÉRIE - TOME $40-2007-\mathrm{N}^{\circ} 3$ 
over $X$, to

$$
\underline{\operatorname{End}}(I) \simeq \prod_{i \leqslant 0} \mathbb{A}_{X}^{n_{i}}
$$

where $n_{i}:=\operatorname{dim}_{k} H H^{i}(T)$ (this uses that the complex of $k$-vector spaces $H H(T)$ is quasiisomorphic to a direct sum of its cohomology groups).

It follows from Proposition 3.20 and Corollary 3.24, that for any saturated dg-category $T$ over a field $k$, the group scheme aut $(T)$ has only countably many connected components. We therefore obtain the following more precise statement.

COROLLARY 3.26. - Let $T$ be a dg-category saturated over a field $k$. Then, the group scheme $\operatorname{aut}(T)$ is an extension

$$
1 \rightarrow \operatorname{aut}(T)_{e} \rightarrow \operatorname{aut}(T) \rightarrow \Gamma \rightarrow 1,
$$

where $\Gamma$ is a countable discrete group, and aut $(T)_{e}$ is an algebraic group of finite type over $k$.

Proof. - This follows from the fact that $\operatorname{aut}(T)$ has only countably many connected components, and by the fact a connected group scheme locally of finite type over a field $k$ is quasi-compact (see [26, Exp. $\mathrm{VI}_{\mathrm{B}}$, Cor. 3.6]).

\subsection{Two examples}

To finish this section, we will present two fundamental examples of dg-categories of finite type coming from algebraic geometry and representation theory. They will give by Theorem 3.6 two locally geometric $D^{-}$-stacks, one classifying perfect complexes on a smooth and proper scheme, and a second one classifying complexes of representation of a finite Quiver. They both seem to us important examples to study in the future.

Let $p: X \rightarrow$ Spec $k$ be a smooth and proper morphism of schemes. We consider the category $Q \operatorname{Coh}(X)$ of quasi-coherent sheaves on $X$, as well as $C Q \operatorname{Coh}(X)$ the category of unbounded complexes in $Q \operatorname{Coh}(X)$. As the scheme $X$ is quasi-compact and separated, it is known by [10] that $C Q \operatorname{Coh}(X)$ is a model category for which cofibrations are monomorphisms and equivalences are quasi-isomorphisms. This model category has a natural enrichment in $C(k)$, by tensoring a complex of quasi-coherent sheaves by a complex of $k$-modules. This makes $C Q \operatorname{Coh}(X)$ into a $C(k)$-model category, and we set

$$
L_{\mathrm{qcoh}}(X):=\operatorname{Int}(C Q \operatorname{Coh}(X)) .
$$

Note that $L_{\mathrm{qcoh}}(X)$ is only a $\mathbb{V}$-small dg-category, but with $\mathbb{U}$-small complexes of morphisms between two fixed objects. We denote by $L_{\mathrm{pe}}(X)$ the full sub-dg-category of $L_{\mathrm{qcoh}}(X)$ consisting of perfect complexes. The dg-category $L_{\mathrm{pe}}(X)$ is still only $\mathbb{V}$-small, but is quasiequivalent to a $\mathbb{U}$-small dg-category as the set of isomorphisms classes of objects in $\left[L_{\mathrm{pe}}(X)\right]$ is $\mathbb{U}$-small. We will therefore do as if $L_{\mathrm{pe}}(X)$ were a $\mathbb{U}$-small dg-category.

The triangulated category $\left[L_{\mathrm{qcoh}}(X)\right]$ is naturally equivalent to the unbounded derived category $D_{\mathrm{qcoh}}(X)$ of quasi-coherent sheaves on $X$. In the same way, $\left[L_{\mathrm{pe}}(X)\right]$ is naturally equivalent to $D_{\mathrm{pe}}(X)$, the full sub-category of $D_{\mathrm{qcoh}}(X)$ consisting of perfect complexes. By [3], we know that $D_{\mathrm{pe}}(X)$ is precisely the sub-category of compact objects in $D_{\mathrm{qcoh}}(X)$, and furthermore that $D_{\mathrm{qcoh}}(X)$ is compactly generated. This easily implies that the restricted Yoneda embedding

$$
L_{\mathrm{qcoh}}(X) \rightarrow \widehat{L_{\mathrm{pe}}(X)}
$$


is a quasi-equivalence. Furthermore, $L_{\mathrm{pe}}(X)$ is a triangulated dg-category, and therefore

$$
\left.L_{\mathrm{pe}}(X) \simeq \widehat{L_{\mathrm{pe}}(X}\right)_{\mathrm{pe}} \simeq\left(L_{\mathrm{qcoh}}(X)\right)_{\mathrm{pe}} .
$$

LEMMA 3.27. - The dg-category $L_{\mathrm{pe}}(X)$ is saturated.

Proof. - We already know that $L_{\mathrm{pe}}(X)$ is triangulated. As the scheme $X$ is proper and flat over Speck, for two perfect complexes $E$ and $F$ on $X$, the complex of $k$-modules $\mathbb{R} \underline{\operatorname{Hom}}(E, F)$ is perfect. This implies that $L_{\mathrm{pe}}(X)$ is locally perfect. By [3], it is also known that $L_{\mathrm{qcoh}}(X)=\widehat{L_{\mathrm{pe}}(X)}$ has a compact generator, and thus that $L_{\mathrm{pe}}(X)$ is a proper dg-category. Finally, by [30, §8.3], we have $L_{\mathrm{pe}}(X){\widehat{\otimes \mathbb{L}} L_{\mathrm{pe}}}(X)^{\mathrm{op}} \simeq L_{\mathrm{qcoh}}\left(X \times_{k} X\right)$, and the diagonal $\left(L_{\mathrm{pe}}(X)^{\mathrm{op}} \otimes^{\mathbb{L}} L_{\mathrm{pe}}(X)\right)$-dg-module

$$
\begin{aligned}
\delta: L_{\mathrm{pe}}(X)^{\mathrm{op}} \otimes^{\mathbb{L}} L_{\mathrm{pe}}(X) & \rightarrow C(k) \\
(x, y) & \mapsto L_{\mathrm{pe}}(X)(x, y)
\end{aligned}
$$

corresponds by this quasi-equivalence to $\Delta_{X}$, the class of the diagonal in $L_{\mathrm{qcoh}}\left(X \times_{k} X\right)$. As $X$ is smooth and separated over Spec $k$, we know that $\Delta_{X}$ is a perfect complex on $X \times_{k} X$, and thus lies in $L_{\mathrm{qcoh}}\left(X \times_{k} X\right)_{\mathrm{pe}}$. By definition, this means that $L_{\mathrm{pe}}(X)$ is a smooth dg-category.

Definition 3.28. - The $D^{-}$-stack $\mathcal{M}_{L_{\mathrm{pe}}(X)}$ is called the $D^{-}$-stack of perfect complexes on $X$. It is denoted by $\mathbb{R} \underline{\operatorname{Perf}}(X)$, and its truncation by $\underline{\operatorname{Perf}}(X):=t_{0}(\underline{\operatorname{Perf}}(X))$.

For any commutative $k$-algebra $A$, the simplicial set $\underline{\mathbb{R e r f}}(X)(A)$ is by definition $\operatorname{Map}_{d g-C a t}\left(L_{\mathrm{pe}}(X)^{\mathrm{op}}, \widehat{A}_{\mathrm{pe}}\right)$, which in turn is equivalent to the nerve of the category of quasiisomorphisms between perfect complexes on $X \times^{h}$ Spec $A$ (see $[30, \S 8.3]$ ). In other words, if $\mathbb{R}$ Perf denotes the $D^{-}$-stack $\mathcal{M}_{1}$, which is the $D^{-}$-stack of perfect complexes, we have

$$
\mathbb{R} \underline{\operatorname{Perf}}(X) \simeq \operatorname{Map}(X, \underline{\mathbb{R} P e r f}),
$$

where Map denotes the internal Hom of the category $S t(k)$ (see [35]). This justifies the terminology of Definition 3.28. In particular, the truncation $\operatorname{Perf}(X):=t_{0} \mathbb{R} \operatorname{Perf}(X)$ is the (un-derived) stack of perfect complexes on $X$, whose absolute version has been considered previously in $[8, \S 21]$.

Lemma 3.27 and Theorem 3.6 imply the following corollary.

COROLLARY 3.29. - The $D^{-}$-stack $\mathbb{R} \operatorname{Perf}(X)$ is locally geometric and locally of finite type. For any global point $E \in \mathbb{R} \operatorname{Perf}(X)(k)$, we have

$$
\mathbb{T}_{\mathbb{R} \operatorname{Perf}(X), E} \simeq \underline{\mathbb{H o m}}(E, E)[1] .
$$

Remark 3.30.-

1. From Corollaries 3.21, 3.22 and 3.29 we find a new proof of the existence of the Artin 1-stack $\operatorname{Coh}(X)$ of coherent sheaves on a smooth and proper scheme (e.g. as presented in [17]). More generally, we also find a new proof of the existence of the Artin 1-stack of 1-rigid perfect complexes on $X$, previously constructed in [19]. It seems to us important to notice that this new proof uses in an essential way higher stacks, as it is based on the morphism $\pi$ from $\underline{C o h}(X)$ to the stack of perfect complexes, sending a coherent sheaf $E$ on $X$ to the complex $\underline{R} \underline{H o m}\left(E_{0}, E\right)$, for $E_{0}$ a compact generator in $D_{\mathrm{qcoh}}(X)$.

$4^{\text {e }}$ SÉRIE - TOME $40-2007-\mathrm{N}^{\circ} 3$ 
2. When $X$ is no longer proper and smooth, the $D^{-}$-stack $\mathcal{M}_{L_{\mathrm{pe}}(X)}$ only classifies pseudoperfect complexes on $X$. These are the complexes of quasi-coherent sheaves $E$ on $X$ such that for any perfect complexes $F$ on $X$ the complex of $k$-modules $\mathbb{R} \underline{H o m}(F, E)$ is perfect. When $X$ is only assumed to be smooth, then a pseudo-perfect complex is perfect (by 2.8), and when $X$ is only assumed to be proper and flat then perfect complexes are pseudoperfect (by 2.8). In general, the objects classified by $\mathcal{M}_{L_{\mathrm{pe}}(X)}$ have no clear relations with perfect complexes on $X$, and this is the reason why we did not consider this general situation, even if Corollary 3.29 stays most probably correct under much more general assumptions than smoothness and properness.

Let $E \in D_{\mathrm{qcoh}}(X)$ be a compact generator, and let us consider the induced morphism

$$
\pi: \underline{\mathbb{P e r f}}(X) \rightarrow \underline{\mathbb{P} \operatorname{Perf}},
$$

as during the proof of Theorem 3.6. The morphism $\pi$ simply sends a perfect complex $F$ on $X$ to the perfect complex $\mathbb{R} \underline{H o m}(E, F)$. Let $\nu: \mathbb{Z} \rightarrow \mathbb{N}$ be a function with finite support, and let us consider the sub- $D^{-}$-stack $\mathbb{R} \underline{\operatorname{Perf}}(X)^{\nu}$ of $\mathbb{R} \operatorname{Perf}(X)$ defined as in 3.20.

COROLLARY 3.31. - The $D^{-}$-stack $\mathbb{R} \operatorname{Perf}(X)^{\nu}$ is n-geometric for some $n$, and strongly of finite presentation.

Corollaries 3.29 and 3.31 provide in particular a proof of the result [8, Thm. 21.5].

Corollary 3.31 has an interesting consequence when $X$ is a smooth and projective variety over a field $k$, as in this case there is a natural choice for the generator $E$ of $D_{\mathrm{qcoh}}(X)$. Indeed, recall from [3] that there exists an integer $d$ such that the vector bundle $E:=\bigoplus_{i \in[0, d]} \mathcal{O}(-i)$ is a compact generator of $D_{\text {qcoh }}(X)$, where $\mathcal{O}(1)$ is a very ample line bundle on $X$. From Corollary 3.31 we thus get a boundedness criterion for perfect complexes on $X$, which seems to be a generalization of Kleiman criterion. For this, we will say that a family of objects $\left\{E_{i}\right\}_{i \in I}$ in $D_{\mathrm{pe}}(X)$ is bounded, if the corresponding family of points $E_{i} \in \mathbb{R} \operatorname{Parf}(X)(k)$ belongs to an open sub- $D^{-}$-stack which is strongly of finite presentation.

COROLLARY 3.32. - Let $k$ be a field and $X$ be a smooth and projective variety over $k$. Let $\mathcal{O}(1)$ be a very ample line bundle on $X$. Then, there exists an integer $d$, such that the following condition is satisfied:

A family of perfect complexes $\left\{E_{i}\right\}_{i \in I}$ on $X$ is bounded if and only if there exists a function $\nu: \mathbb{Z} \rightarrow \mathbb{N}$ with finite support, such that

$$
\operatorname{Dim}_{k} \mathbb{H}^{k}\left(X, E_{i}(j)\right) \leqslant \nu(k) \quad \forall k \forall j \in[0, d] \forall i \in I .
$$

Proof. - This follows from the fact that any open sub- $D^{-}$-stack strongly of finite presentation of $\mathbb{R} \operatorname{Perf}(X)$ is contained in some $\mathbb{R} \operatorname{Perf}(X)^{\nu}$, together with the fact that for the generator $E:=\bar{\bigoplus}_{i \in[0, d]} \mathcal{O}(-i)$ the morphism

$$
\pi: \underline{\mathbb{P} \operatorname{Perf}}(X) \rightarrow \underline{\mathbb{P} P \operatorname{erf}}
$$

sends a perfect complex $F$ on $X$ to $\bigoplus_{i \in[0, d]} \mathbb{R} \Gamma(X, F(i))$.

We now pass to our second example. Let $B$ be an associative $k$-algebra, which is assumed to be projective and of finite type as a $k$-module. We also assume that $B$ is perfect as a complex of $B \otimes_{k} B^{\mathrm{op}}$-modules. Then, the dg-category with a unique object $B$ is smooth and proper, and thus by Theorem 3.6 the $D^{-}$-stack $\mathcal{M}_{B}$ is locally geometric. By definition, for any 
$A \in s k-C A l g$, there exists a natural equivalence between $\mathcal{M}_{B}(A)$ and the nerve of the category of quasi-isomorphisms between perfect $B \otimes_{k} N(A)$-dg-modules. Therefore, $\mathcal{M}_{B}$ is a moduli for perfect complexes of $B$-modules.

Now, let us assume that $Q$ is an oriented finite Quiver. Let $B=k[Q]^{\text {op }}$ be the opposite $k$-algebra associated to $Q$. The $B^{\mathrm{op}}$-modules are precisely the representations of $Q$ in $k$-modules, and thus the dg-category $\widehat{B}$ can be identified with the dg-category of complexes of representations of $Q$ with coefficients in $k$-modules. The pseudo-perfect objects in $\widehat{B}$ are simply the complexes of representations of $B$ whose underlying complex of $k$-modules is perfect.

Definition 3.33. - With the notations above, the $D^{-}$-stack $\mathcal{M}_{B}$ is called the $D^{-}$-stack of pseudo-perfect complexes of representations of $Q$. It is denoted by $\mathbb{R} \operatorname{Parf}(Q)$.

The $k$-algebra $B$ is easily seen to be homotopically finitely presented in the model category of dg-algebras. If the quiver $Q$ has no loops, then $B$ is furthermore a smooth and proper dg-category. Therefore, Theorem 3.6 implies the following corollary.

COROLLARY 3.34. - The $D^{-}$-stack $\mathbb{R} \operatorname{Parf}(Q)$ is locally geometric and locally of finite type. For a global point $E \in \mathbb{R} \operatorname{Parf}(Q)(k)$, corresponding to a complex of representations of $Q$, we have

$$
T_{\mathbb{R} \text { Parf }}(Q), E \simeq \underline{\mathbb{H o m}}(E, E)[1],
$$

where $\mathbb{R}$ Hom denotes the derived Hom's of complexes of representations of $Q$.

One has a natural projection, corresponding to the natural generator of $\widehat{B}$ (i.e. $B$ itself)

$$
\underline{\mathbb{P a r f}}(Q) \rightarrow \underline{\mathbb{R}} \underline{\underline{P e r f}} .
$$

As in Proposition 3.20, one gets for any $\nu: \mathbb{Z} \rightarrow \mathbb{N}$ with finite support, an open sub- $D^{-}$-stack $\underline{\mathbb{P a r f}}(Q)^{\nu}$.

COROLLARY 3.35. - The $D^{-}$-stack $\mathbb{R} \operatorname{Parf}(Q)^{\nu}$ is n-geometric for some $n$, and strongly of finite presentation.

\section{Acknowledgements}

We are grateful to T. Pantev and L. Katzarkov for motivating discussions concerning possible applications of stacks of perfect complexes on algebraic varieties in order to produce some kind of compactifications of stacks of vector bundles. The present work precisely started when trying to write down a proof of the geometricity of the stack of perfect complexes on a scheme.

We also thank C. Simpson, G. Tabuada and G. Vezzosi for many useful comments on earlier versions of this manuscript as well as for their interests.

\section{REFERENCES}

[1] Anel M., ToËN B., Dénombrabilité des classes d'équivalences dérivées des variétés algébriques, $J$. Algebraic Geom., submitted for publication.

[2] Bondal A., KAPRANOV M., Enhanced triangulated categories, Math. USSR Sbornik 70 (1991) 93 107.

[3] Bondal A., VAN DEN BERGH M., Generators and representability of functors in commutative and non-commutative geometry, Mosc. Math. J. 3 (1) (2003) 1-36.

[4] Ciocan-Fontanine I., Kapranov M., Derived Hilbert schemes, J. Amer. Math. Soc. 15 (4) (2002) 787-815.

$4^{\mathrm{e}}$ SÉRIE - TOME $40-2007-\mathrm{N}^{\circ} 3$ 
[5] GORSKI J., Representability of derived Quot functor, in preparation.

[6] Hinich V., DG coalgebras as formal stacks, J. Pure Appl. Algebra 162 (2-3) (2001) 209-250.

[7] HirschHoRn P., Model Categories and Their Localizations, Math. Surveys and Monographs, vol. 99, Amer. Math. Soc., Providence, 2003.

[8] Hirschowitz A., Simpson C., Descente pour les $n$-champs, math.AG/9807049.

[9] Hovey M., Model Categories, Mathematical Surveys and Monographs, vol. 63, Amer. Math. Soc., Providence, 1998.

[10] Hovey M., Model category structures on chain complexes of sheaves, Trans. Amer. Math. Soc. 353 (6) (2001) 2441-2457.

[11] INABA M., Toward a definition of moduli of complexes of coherent sheaves on a projective scheme, J. Math. Kyoto Univ. 42 (2) (2002) 317-329.

[12] JoYCE D., Configurations in abelian categories. II. Ringel-Hall algebras, Adv. Math. 210 (2) (2007) 635-706.

[13] KAPRANOV M., Injective resolutions of $B G$ and derived moduli spaces of local systems, J. Pure Appl. Algebra 155 (2-3) (2001) 167-179.

[14] KelLER B., On differential graded categories, International Congress of Mathematicians, vol. II, Eur. Math. Soc., Zürich, 2006, pp. 151-190.

[15] Kontsevich M., Enumeration of rational curves via torus actions. The moduli space of curves, in: Progr. Math., vol. 129, Birkhäuser, Boston, MA, 1995, pp. 335-368.

[16] Kontsevich M., Soibelmann Y., Notes on A-infinity algebras, A-infinity categories and noncommutative geometry. I, math.RA/0606241.

[17] Laumon G., Moret-Bailly L., Champs algébriques, A Series of Modern Surveys in Mathematics, vol. 39, Springer-Verlag, 2000.

[18] LaZARev A., Homotopy theory of $A_{\infty}$ ring spectra and applications to $M U$-modules, K-Theory 24 (3) (2001) 243-281.

[19] Lieblich M., Moduli of complexes on a proper morphism, J. Algebraic Geom. 15 (2006) 175-206.

[20] LuRIE J., Derived algebraic geometry, Ph.D. thesis, unpublished, available at http://www.math. harvard.edu/ lurie/.

[21] NeEman A., Triangulated Categories, Annals of Mathematics Studies, vol. 148, Princeton University Press, Princeton, NJ, 2001, viii+449 pp.

[22] REZK C., A model for the homotopy theory of homotopy theories, Trans. Amer. Math. Soc. 353 (3) (2001) 973-1007.

[23] SCHWEde S., SHIPLEy B., Algebras and modules in monoidal model categories, Proc. London Math. Soc. (3) 80 (2000) 491-511.

[24] SCHWEDE S., SHIPLEY B., Stable model categories are categories of modules, Topology 42 (1) (2003) $103-153$.

[25] Schwede S., Shipley B., Equivalences of monoidal model categories, Algebraic Geom. Topol. 3 (2003) 287-334.

[26] Demazure M., Grothendieck A., Schémas en groupes. I: Propriétés générales des schémas en groupes (SGA 3-1), in: Lecture Notes in Mathematics, vol. 151, Springer-Verlag, Berlin-New York, 1970, xv+564 pp.

[27] SiMPSON C., Algebraic (geometric) $n$-stacks, math.AG/9609014.

[28] TABUADA G., Une structure de catégorie de modèles de Quillen sur la catégorie des dg-catégories, C. R. Acad. Sci. Paris 340 (2005) 15-19.

[29] Thomas R., A holomorphic Casson invariant for Calabi-Yau 3-folds, and bundles on $K 3$ fibrations, J. Differential Geom. 54 (2) (2000) 367-438.

[30] ToËN B., The homotopy theory of dg-categories and derived Morita theory, Invent. Math. 167 (3) (2007) 615-667.

[31] Tö̈N B., Derived Hall algebras, Duke Math. J. 135 (3) (2006) 587-615.

[32] ToËN B., Higher and derived stacks: a global overview, math.AG/0604504.

[33] ToËn B., VAQuiÉ M., Algébrisation des variétés analytiques complexes et catégories dérivées, math.AG/0703555.

[34] ToËn B., Vezzosi G., Homotopical algebraic geometry I: Topos theory, Adv. in Math. 193 (2005) $257-372$. 
[35] ToËn, B., Vezzosi, G., Homotopical algebraic geometry II: Geometric stacks and applications, Mem. Amer. Math. Soc., in press.

[36] ToËn B., Vezzosi G., From HAG to DAG: derived moduli spaces, in: J.P.C. Greenlees (Ed.), Axiomatic, Enriched and Motivic Homotopy Theory, Proceedings of the NATO Advanced Study Institute, Cambridge, UK (9-20 September 2002), in: NATO Science Series II, vol. 131, Kluwer, 2004, pp. 175-218.

(Manuscrit reçu le 4 juillet 2006;

accepté, après révision, le 3 mai 2007.)

Bertrand TOËN

Laboratoire Emile Picard, Bât 1R2,

Université Paul Sabatier, 31062 Toulouse Cedex 9, France E-mail: toen@math.ups-tlse.fr

Michel VaquiÉ Laboratoire Emile Picard, Bât 1R2,

Université Paul Sabatier, 31062 Toulouse Cedex 9, France E-mail: vaquie@math.ups-tlse.fr 